\title{
Raman and IR Spectral and DFT Based Vibrational and Electronic Characterization of Isolated and Zwitterionic Forms of L-Tyrosine
}

\author{
Yadav RA ${ }^{1 *}$, Dixit $\mathbf{V}^{1}$, Yogesh $\mathbf{M}^{2}$ and Santhosh $\mathbf{C}^{2}$ \\ ${ }^{1}$ Laser and Spectroscopy Laboratory, Department of Physics, Banaras Hindu University, Varanasi -221005(UP), India \\ ${ }^{2}$ Department of Atomic and Molecular Physics, Manipal University, Manipal-576104 (Karnatak), India
}

\begin{abstract}
Comparative structural and vibrational investigations in two different forms of the L-Tyrosine (L-TYR) have been carried out using Raman and IR spectral and DFT methods. For the optimized structures of the most stable conformers the vibrational assignments of the experimental IR and Raman bands have been proposed using the results of the DFT-B3LYP computations and the PEDs computed using GAR2PED software. The optimized geometrical structures of the molecule belong to the $\mathrm{C} 1$ point group in the zwitterionic and isolated forms of the molecule. Possibility of the charge transfer phenomena in the molecule has been investigated in light of the HOMO-LUMO analysis. The electron density mappings of the iso-surfaces with the molecular electrostatic potential (MEP) have been carried out to obtain the different information associated with the size, shape, charge density distribution and site of chemical reactivity of the molecule. On the basis of the NBO analysis presence of intramolecular $\mathrm{H}$ bonding and intramolecular charge transfer (ICT) have been proposed.
\end{abstract}

Keywords: Optimized Geometry; IR and Raman spectra; Frontier orbitals; MEP; NBO; L-Tyrosine; vibrational assignments

\section{Introduction}

L-Tyrosine (L-TYR), is one of the essential amino acids and an important component of various food products like milk, fish, cheese, peanuts, almonds, chicken, etc. It acts as a potent anti-depressant agent and also enhances the cellular strength of immune systems in the human body. The phenolic-OH group in the tyrosine molecule is essential for stimulating the antibacterial activity. It is precursor of number of hormones and neurotransmitters [1]. In recent years much attention is being paid towards investigations of the natural and safer antioxidant compounds due to their crucial roles in the food, cosmetics and pharmaceutical products [2]. Also, these compounds enhance the shelf life and are used for prevention of the age related diseases by retarding the process of the lipid per-oxidation and chain reaction [3].

L-TYR helps to regulate the mood, stimulate the nervous system and support the creation of the neurotransmitters including the dopamine, nor-epinephrine and epinephrine [4]. These amino acids also play essential role in the synthesis of melanin which protects against harmful effects of the UV radiations [5] and, also found to be used for the treatment of the allergies, headaches and Parkinson's disease. Their usefulness for the normal functioning of the thyroid, pituitary, and adrenal glands is crucial for living bodies. Thus, L-TYR can affect the health, together with the other amino acids, it may have very important effects, in the people facing dementia, including Alzheimer's disease and it may also reduce the adverse effects of the environmental, psychosocial, and physical stress [6-9]. Recently, some researches about tyrosine and its enzymes have been carried out using molecular dynamics simulations [10-12]. In recent years, the crystal and molecular structures of L-TYR $[13,14]$ and also numerous enzymes associated with tyrosine, e.g., tyrosine phosphatise [15-19] have been investigated. Several workers $[4,6,20-24]$ have investigated the tyrosine molecule and its derivatives using different spectroscopic techniques. However, detailed structural and vibrational characteristics of the L-TYR molecule have been investigated by Contreras et al. [6]. But, Contreras et al. [6] assigned only the functional group modes and only few of the skeletal modes (finger print modes) and excluded the explanation of the weak IR band at $2075 \mathrm{~cm}^{-1}$. Also, HOMO-LUMO orbitals and electron density and molecular electroscopic potentials plots and contours studies, which provide important information about the reactivity, hardness and other characteristics of the molecule, do not appear to have been investigated so far.

Therefore, in the present article we have computed the optimized structural parameters, natural, APT and Mulliken atomic charges and the fundamental vibrational frequencies along with the IR intensities, Raman activities and depolarization ratios of the Raman bands employing the DFT-B3LYP method using the $6-311++G^{* *}$ basis set (Gaussian-09 software) [25,26] for the lowest energy conformers of L-TYR. The experimentally observed IR and Raman frequencies have been analysed with the help of visualization of the form of the normal mode using the Gauss View software and the corresponding PEDs available from the GAR2PED software [25-29]. Reactivity of the title molecule was investigated using the HOMO-LUMO analysis and their orbital energies, molecular electrostatic potential. The natural bond orbital analysis (NBO) analysis has also been carried out.

\section{Computational Details}

The geometrical structure of the most stable conformer of ethyl benzene was optimized with the help of the Gauss View 5.0 and

*Corresponding author: Yadav RA, Laser and Spectroscopy Laboratory Department of Physics, Banaras Hindu University, Varanasi -221005(UP), India, Tel: +91 542 2368593; Fax: +91 542 2368390; E-mail: rayadav@bhu.ac.in, ray1357@gmail.com

Santhosh C, Department of Atomic and Molecular Physics, Manipal University, Manipal-576104 (Karnatak), India, Tel: 0820-2925071, E-mail: santhosh.cls@ manipal.edu

Received August 13, 2015; Accepted October 08, 2015; Published October 12, 2015

Citation: Yadav RA, Dixit V, Yogesh M, Santhosh C (2015) Raman and IR Spectral and DFT Based Vibrational and Electronic Characterization of Isolated and Zwitterionic Forms of L-Tyrosine. Pharm Anal Acta 6: 439. doi:10.4172/21532435.1000439

Copyright: @ 2015 Yadav RA et al. This is an open-access article distributed under the terms of the Creative Commons Attribution License, which permits unrestricted use, distribution, and reproduction in any medium, provided the original author and source are credited. 
Citation: Yadav RA, Dixit V, Yogesh M, Santhosh C (2015) Raman and IR Spectral and DFT Based Vibrational and Electronic Characterization of Isolated and Zwitterionic Forms of L-Tyrosine. Pharm Anal Acta 6: 439. doi:10.4172/21532435.1000439

Gaussian 09 software as elaborated in ref. [25-28]. By replacing the two $\mathrm{H}$-atoms of the $\mathrm{CH}_{3}$ group with the $-\mathrm{NH}_{2}$ and $-\mathrm{COOH}$ moieties the resulting structures were again optimized. The minimum energy structure corresponds to the most stable structure of L- Phenylalanine (L-PHAL). With this structure of L-PHAL by replacing the $\mathrm{H}$ atom of the para position relative to the side carbon chain by an $\mathrm{OH}$ group we again optimized the structures and selected the minimum energy structure which is the desired structures of L-TYR (Figure 1). The relative and total energies of the most probable conformers have been presented in Table 1 (zwitterionic form) and Table 2 (isolated form). The vibrational spectrum and related parameters have been investigated in isolated as well as zwitterionic forms of the most stable conformer of L-TYR (Figure 1) (replacing the $-\mathrm{COOH}$ and $-\mathrm{NH}_{2}$ groups by the $-\mathrm{CO}_{2}$ and $-\mathrm{NH}_{3}$ groups and optimize again).

The unscaled B3LYP/6-311++ $\mathrm{G}^{* *}$ vibrational wave numbers are usually slightly larger than those of the observed values; therefore, for the sake of the reasonable frequency matching we employed the quantum chemical scaling [30]. The assignments of all the normal modes of vibration have been proposed with the help of the PEDs, calculated using the GAR2PED software [25-29] and with the help of Gauss View software by observing the modes of vibration corresponding to the computed fundamentals.

The molecular electrostatic potential (MEP) surfaces predict the sites, the relative reactivity towards the electrophilic attack, the biological recognition and the hydrogen bonding interactions. The NBO analysis has also been carried out to investigate the intra-molecular charge transfer (CT) interactions, re-hybridisation, the delocalization of electron density (ED) and also second order perturbation theory of molecular orbitals within the present molecule in both the situations.

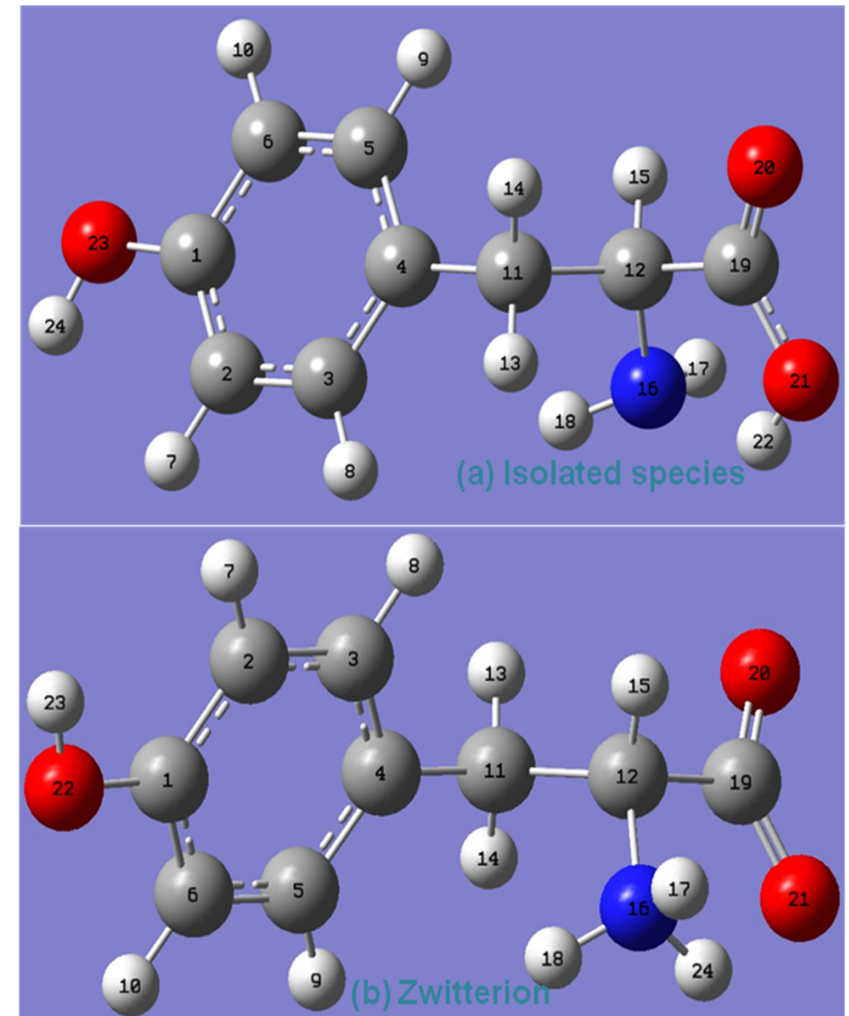

Figure 1: Optimized structures of lowest energy conformers of the isolated and zwitterionic form of $L$ Tyrosine molecule.

\section{Experimental Detail}

The compound L- Tyrosine was purchased from the Sigma Aldrich Chemical Co. (USA). A little amount (1-2 mg) of pure, lyophilized powder of L-TYR, was used to record the Raman spectra using a home assembled micro-Raman spectrometer employing Horiba Jobin-Yuon Spectrometer (iHR-320) system with an inverted microscope (Nikon Eclipse Ti-U, Japan). A Diode laser (Star Bright Diode Laser, Torsana Laser Tech, Denmark) $\lambda 785 \mathrm{~nm}$ was used as the source to illuminate the sample sandwiched between quartz cover slip and borosilicate glass slide. The sample was mounted on the microscope stage and a $60 \mathrm{X}$ microscope objective (a converging lens) was used to focus the laser beam and to collect the Raman signals. A liquid nitrogen cooled Symphony CCD detector was employed to collect the Raman scattered radiations. The spectral range of the Raman spectrum was 200-3100 $\mathrm{cm}^{-1}$ with the resolution $5 \mathrm{~cm}^{-1}$ and accuracy $2 \mathrm{~cm}^{-1}$ at spectrometer slit width $100 \mu \mathrm{m}$. In order to achieve a better Raman spectra data were obtained using a laser power of $47 \mathrm{~mW}$ with the acquisition times of 4 min.

FTIR spectrum of L-TYR sample was recorded using KBr pellet. $10 \mathrm{mg}$ of the sample was properly mixed with $990 \mathrm{mg} \mathrm{KBr}$. A pellet from this mixture was made applying appropriate pressure for thirty minutes. FTIR spectrum was recorded using the above pellet at around $23^{\circ} \mathrm{C}$ temperatures, using an FTIR spectrometer (Jasco 6300) with a standard source. The spectra were recorded in the range $400-4000 \mathrm{~cm}$ ${ }^{1}$. We have taken 200 scans with $2 \mathrm{~cm}^{-1}$ spectral resolution.

\section{Results and Discussion}

\section{Molecular geometry}

Front and side views of the geometrical structures of L-TYR have been given in the figure 1 . Table 1 contains all the geometrical parameters of L-TYR. It is to be noted that the bond lengths computed at the B3LYP/6-311++ $\mathrm{G}^{\star *}$ level appear to be slightly larger in isolated molecule as compared to the observed ones while in zwitterionic form some bond lengths are larger while others are shorter than those of the observed values [6,31]. The atoms $\mathrm{C}_{1}, \mathrm{C}_{2}, \mathrm{C}_{3}, \mathrm{C}_{4}, \mathrm{C}_{5}, \mathrm{C}_{6}, \mathrm{C}_{11}, \mathrm{H}_{7}, \mathrm{H}_{8}, \mathrm{H}_{9}$, $\mathrm{H}_{10}, \mathrm{O}_{23}$ and $\mathrm{H}_{24}$ are coplanar with the phenyl ring plane. The atoms $\mathrm{C}_{11}$ and $\mathrm{C}_{12}$ are tetrahedrally connected to the atoms $\mathrm{C}_{4}, \mathrm{C}_{12}, \mathrm{H}_{13}, \mathrm{H}_{14}$ and $\mathrm{C}_{19}, \mathrm{C}_{11}, \mathrm{~N}_{16}, \mathrm{H}_{17}$ respectively, the L-TYR molecule is found to have no symmetry. The computed and observed $\mathrm{CH}$ bond lengths are found to have too large differences (Table 1) for isolated molecule while these agree for zwitterionic form. In the phenyl ring, CC bond lengths associated with the $\mathrm{C}$ atom directly attached to the side chain with two functional groups are larger, significantly in zwitterionic form, than the other phenyl ring CC bond lengths. Clearly, the lengths of CC $\sigma$-bonds in the side chain are much larger compared to the CC bond lengths in the phenyl ring. The $\mathrm{C}_{4}-\mathrm{C}_{11}$ bond has been found to be the shortest while the $\mathrm{C}_{11}-\mathrm{C}_{12}$ bond in isolated and $\mathrm{C}_{12}-\mathrm{C}_{19}$ bond in zwitterion have been found the largest of all the $\mathrm{CC} \sigma$-bonds in the side chain of the optimized L-TYR molecule (Figure 1). The $\mathrm{O}_{23}-\mathrm{H}_{24}$ bond length in isolated form is found to be the shortest of all the bonds (Table 3). In zwitterionic form, the bond containing $\mathrm{H}_{24}$ atom is exceptionally larger than those of other two $\mathrm{NH}$ bonds of the $\mathrm{NH}_{3}$ group (Table 3). The computed bond angles are almost in agreement with the observed data, except those which are either associated with the functional groups or with the higher electronegative atoms. The bond angle $\alpha\left(\mathrm{O}_{20}-\mathrm{C}_{19}-\mathrm{O}_{21}\right)$ is the largest angle in the molecule. It is interesting to note that the differences between the computed and observed bond angles $\alpha\left(\mathrm{H}_{15}\right.$ $\left.\mathrm{C}_{12}-\mathrm{N}_{16}\right), \alpha\left(\mathrm{C}_{12}-\mathrm{N}_{16}-\mathrm{H}_{18}\right), \alpha\left(\mathrm{O}_{20}-\mathrm{C}_{19}-\mathrm{O}_{21}\right), \alpha\left(\mathrm{C}_{12}-\mathrm{C}_{19}-\mathrm{O}_{21}\right), \alpha\left(\mathrm{C}_{11}-\mathrm{C}_{12}-\mathrm{C}_{19}\right)$, $\alpha\left(\mathrm{C}_{12}-\mathrm{C}_{19}-\mathrm{O}_{20}\right)$ and $\alpha\left(\mathrm{C}_{12}-\mathrm{C}_{11}-\mathrm{H}_{14}\right)$ are $5.3,3.8,3.3,2.9,2.8,2.4$ and 
Citation: Yadav RA, Dixit V, Yogesh M, Santhosh C (2015) Raman and IR Spectral and DFT Based Vibrational and Electronic Characterization of Isolated and Zwitterionic Forms of L-Tyrosine. Pharm Anal Acta 6: 439. doi:10.4172/21532435.1000439

Page 3 of 18

\begin{tabular}{|c|c|c|c|c|c|c|c|c|}
\hline \multirow[t]{2}{*}{ Con. } & \multirow[t]{2}{*}{$\mathrm{E}_{\mathrm{T}}$ (Hartree) } & \multicolumn{5}{|c|}{$E_{R}$} & \multirow{2}{*}{$\begin{array}{c}\mu \\
\text { (Debye) }\end{array}$} & \multirow[t]{2}{*}{ Point Group } \\
\hline & & Hartree & $\left(x 10^{-2}\right) \mathrm{eV}$ & Cal/mole & $\mathrm{cm}^{-1}$ & Kelvin & & \\
\hline $\mathrm{C}-1$ & -630.22675940 & 0.00000 & 0.0 & 0.0 & 0.0 & 0.000000 & 12.9889 & $\mathrm{C}_{1}$ \\
\hline $\mathrm{C}-2$ & -630.22631622 & 0.00044318 & 1.206 & 278.10 & 97.267 & 139.946 & 14.6863 & $\mathrm{C}_{1}$ \\
\hline C-3 & -630.22326532 & 0.00349408 & 9.508 & 2192.569 & 766.862 & 1103.350 & 12.1899 & $\mathrm{C}_{1}$ \\
\hline
\end{tabular}

Con. $\rightarrow$ Conformer, $\mathrm{E}_{\mathrm{T}} \rightarrow$ Total energy, $\mathrm{E}_{\mathrm{R}} \rightarrow$ Relative energy and $\mu \rightarrow$ Dipole moment.

Table 1: Total and relative energies of the possible conformers of zwitterionic L-TYR.

\begin{tabular}{|c|c|c|c|c|c|c|c|}
\hline \multirow[t]{2}{*}{ Con. } & \multirow[t]{2}{*}{$\mathrm{E}_{\mathrm{T}}$ (Hartree) } & \multicolumn{5}{|c|}{$E_{R}$} & \multirow[t]{2}{*}{ Point Group } \\
\hline & & Hartree & $\left(x 10^{-2}\right) \mathrm{eV}$ & Kcal/mole & $\mathbf{c m}^{-1}$ & Kelvin & \\
\hline C-1 & -630.21003038 & 0.00000 & 0.0 & 0.0 & 0.0 & 0.000000 & $\mathrm{C}_{1}$ \\
\hline C-2 & -630.20763457 & 0.00239581 & 6.52 & 1.5034 & 525.82 & 756.54 & $\mathrm{C}_{1}$ \\
\hline C-3 & -630.2075066 & 0.00252378 & 6.87 & 1.5837 & 553.90 & 796.95 & $\mathrm{C}_{1}$ \\
\hline C-4 & -630.20734022 & 0.00269016 & 7.32 & 1.6881 & 590.42 & 849.49 & $C_{1}$ \\
\hline C-5 & -630.20656242 & 0.00346796 & 9.44 & 2.1762 & 761.13 & 1095.10 & $\mathrm{C}_{1}$ \\
\hline $\mathrm{C}-6$ & -630.20637205 & 0.00368533 & 10.03 & 2.3126 & 808.84 & 1163.74 & $C_{1}$ \\
\hline $\mathrm{C}-7$ & -630.20591794 & 0.00411294 & 11.19 & 2.5809 & 902.68 & 1298.77 & $\mathrm{C}_{1}$ \\
\hline C-8 & -630.20549309 & 0.00453729 & 12.35 & 2.8472 & 995.82 & 1432.77 & $\mathrm{C}_{1}$ \\
\hline C-9 & -630.20499927 & 0.00503111 & 13.69 & 3.1571 & 1104.20 & 1588.71 & $C_{1}$ \\
\hline$C-10$ & -630.20417585 & 0.00585453 & 15.93 & 3.6738 & 1284.92 & 1848.72 & $\mathrm{C}_{1}$ \\
\hline C-11 & -630.20161342 & 0.00841646 & 22.90 & 5.2814 & 1847.20 & 2657.72 & $\mathrm{C}_{1}$ \\
\hline C-12 & -630.19973750 & 0.01029288 & 28.01 & 6.4589 & 2259.03 & 3250.26 & $C_{1}$ \\
\hline$C-13$ & -630.19862017 & 0.01141021 & 31.05 & 7.1600 & 2504.25 & 3603.08 & $\mathrm{C}_{1}$ \\
\hline C-14 & -630.19794310 & 0.01208728 & 32.89 & 7.5849 & 2652.85 & 3816.89 & $\mathrm{C}_{1}$ \\
\hline C-15 & -630.19568471 & 0.01434567 & 39.04 & 9.0020 & 3148.51 & 4530.03 & $C_{1}$ \\
\hline
\end{tabular}

Con. $\rightarrow$ Conformer, $\mathrm{E}_{\mathrm{T}} \rightarrow$ Total energy, $\mathrm{E}_{\mathrm{R}} \rightarrow$ Relative energy.

Table 2: Thermo-molecular parameters of the conformer $(C-1)$ of zwitterionic L-TYR.

\begin{tabular}{|c|c|c|c|c|}
\hline \multirow{2}{*}{$\begin{array}{c}\text { Parameters } \\
\text { (Bond lengths, Angles) }\end{array}$} & \multicolumn{2}{|c|}{ B3LYP Values (Computed) } & \multicolumn{2}{|c|}{ Observed Values } \\
\hline & Isolated & $Z w$ & Ref.-31 & Ref.-6 \\
\hline$r\left(C_{1}-C_{2}\right)$ & 1.393 & 1.398 & 1.382 & -- \\
\hline$r\left(C_{1}-C_{6}\right)$ & 1.397 & 1.394 & 1.374 & -- \\
\hline $\mathrm{r}\left(\mathrm{C}_{1}-\mathrm{O}_{22}\right)$ & 1.368 & 1.369 & -- & 1.367 \\
\hline $\mathrm{r}\left(\mathrm{C}_{2}-\mathrm{C}_{3}\right)$ & 1.396 & 1.391 & 1.391 & -- \\
\hline $\mathrm{r}\left(\mathrm{C}_{2}-\mathrm{H}_{7}\right)$ & 1.086 & 1.085 & 0.950 & -- \\
\hline$r\left(C_{3}-C_{4}\right)$ & 1.397 & 1.401 & -- & 1.398 \\
\hline $\mathrm{r}\left(\mathrm{C}_{3}-\mathrm{H}_{8}\right)$ & 1.086 & 1.085 & -- & 1.085 \\
\hline $\mathrm{r}\left(\mathrm{C}_{4}-\mathrm{C}_{5}\right)$ & 1.403 & 1.400 & 1.390 & -- \\
\hline$r\left(C_{4}-C_{11}\right)$ & 1.512 & 1.515 & -- & 1.539 \\
\hline$r\left(C_{5}-C_{6}\right)$ & 1.388 & 1.394 & 1.388 & -- \\
\hline $\mathrm{r}\left(\mathrm{C}_{5}-\mathrm{H}_{9}\right)$ & 1.086 & 1.086 & 0.950 & -- \\
\hline $\mathrm{r}\left(\mathrm{C}_{6}-\mathrm{H}_{10}\right)$ & 1.083 & 1.084 & 0.950 & -- \\
\hline$r\left(C_{11}-C_{12}\right)$ & 1.548 & 1.539 & -- & 1.539 \\
\hline$r\left(\mathrm{C}_{11}-\mathrm{H}_{13}\right)$ & 1.095 & 1.092 & -- & 1.088 \\
\hline $\mathrm{r}\left(\mathrm{C}_{11}-\mathrm{H}_{14}\right)$ & 1.092 & 1.094 & -- & 1.094 \\
\hline $\mathrm{r}\left(\mathrm{C}_{12}-\mathrm{H}_{15}\right)$ & 1.095 & 1.088 & -- & 1.094 \\
\hline$r\left(C_{12}-N_{16}\right)$ & 1.472 & 1.512 & -- & 1.488 \\
\hline$r\left(C_{12}-C_{19}\right)$ & 1.541 & 1.564 & -- & 1.529 \\
\hline $\mathrm{r}\left(\mathrm{N}_{16}-\mathrm{H}_{17}\right)$ & 1.015 & 1.020 & -- & 1.015 \\
\hline $\mathrm{r}\left(\mathrm{N}_{16}-\mathrm{H}_{18}\right)$ & 1.015 & 1.022 & -- & 1.048 \\
\hline $\mathrm{r}\left(\mathrm{N}_{16}-\mathrm{H}_{24}\right)$ & -- & 1.045 & -- & -- \\
\hline $\mathrm{r}\left(\mathrm{C}_{19}-\mathrm{O}_{20}\right)$ & 1.203 & 1.243 & -- & 1.242 \\
\hline$r\left(\mathrm{C}_{19}-\mathrm{O}_{21}\right)$ & 1.342 & 1.261 & -- & 1.259 \\
\hline $\mathrm{r}(\mathrm{OH}) / \mathrm{OH}$ & 0.963 & 0.965 & -- & 0.981 \\
\hline $\mathrm{r}(\mathrm{OH}) / \mathrm{COOH}$ & 0.983 & -- & -- & -- \\
\hline$\alpha\left(C_{2}-C_{1}-C_{6}\right)$ & 119.7 & 119.8 & 120.9 & -- \\
\hline$\alpha\left(C_{2}-C_{1}-O_{22}\right)$ & 123.0 & 122.5 & -- & 121.8 \\
\hline$\alpha\left(C_{6}-C_{1}-O_{22}\right)$ & 117.3 & 117.6 & -- & 117.9 \\
\hline$\alpha\left(C_{1}-C_{2}-C_{3}\right)$ & 119.8 & 119.8 & 119.9 & -- \\
\hline$a\left(C_{1}-C_{2}-H_{7}\right)$ & 120.2 & 120.0 & 120.1 & -- \\
\hline
\end{tabular}


Citation: Yadav RA, Dixit V, Yogesh M, Santhosh C (2015) Raman and IR Spectral and DFT Based Vibrational and Electronic Characterization of Isolated and Zwitterionic Forms of L-Tyrosine. Pharm Anal Acta 6: 439. doi:10.4172/21532435.1000439

Page 4 of 18

\begin{tabular}{|c|c|c|c|c|}
\hline$\alpha\left(C_{3}-C_{2}-H_{7}\right)$ & 120.0 & 120.1 & 120.1 & -- \\
\hline$\alpha\left(C_{2}-C_{3}-C_{4}\right)$ & 121.4 & 121.3 & 121.1 & -- \\
\hline$\alpha\left(C_{2}-C_{3}-H_{8}\right)$ & 118.9 & 118.7 & 119.5 & -- \\
\hline$\alpha\left(C_{4}-C_{3}-H_{8}\right)$ & 119.7 & 119.9 & 119.5 & -- \\
\hline$\alpha\left(C_{3}-C_{4}-C_{5}\right)$ & 117.7 & 117.9 & 118.2 & -- \\
\hline$\alpha\left(C_{3}-C_{4}-C_{11}\right)$ & 121.4 & 121.2 & 120.7 & -- \\
\hline$\alpha\left(C_{5}-C_{4}-C_{11}\right)$ & 120.9 & 120.9 & 120.9 & -- \\
\hline$\alpha\left(C_{4}-C_{5}-C_{6}\right)$ & 121.6 & 121.5 & 120.8 & -- \\
\hline$\alpha\left(C_{4}-C_{5}-H_{9}\right)$ & 119.5 & 119.6 & 119.6 & -- \\
\hline$\alpha\left(C_{6}-C_{5}-H_{9}\right)$ & 118.9 & 118.8 & 119.6 & -- \\
\hline$\alpha\left(C_{1}-C_{6}-C_{5}\right)$ & 119.8 & 119.6 & 120.1 & -- \\
\hline$\alpha\left(C_{1}-C_{6}-H_{10}\right)$ & 119.0 & 119.5 & 119.9 & -- \\
\hline$\alpha\left(C_{5}-C_{6}-H_{10}\right)$ & 121.2 & 120.8 & 119.9 & -- \\
\hline$\alpha\left(C_{4}-C_{11}-C_{12}\right)$ & 113.4 & 114.2 & -- & 114.5 \\
\hline$\alpha\left(C_{4}-C_{11}-H_{13}\right)$ & 109.8 & 110.7 & 109.6 & -- \\
\hline$\alpha\left(C_{4}-C_{11}-H_{14}\right)$ & 110.6 & 109.7 & 109.6 & -- \\
\hline$\alpha\left(C_{12}-C_{11}-H_{13}\right)$ & 108.6 & 106.1 & 109.6 & -- \\
\hline$\alpha\left(C_{12}-C_{11}-H_{14}\right)$ & 107.2 & 109.1 & 109.6 & -- \\
\hline$\alpha\left(\mathrm{H}_{13}-\mathrm{C}_{11}-\mathrm{H}_{14}\right)$ & 107.0 & 106.8 & 108.1 & -- \\
\hline$\alpha\left(C_{11}-C_{12}-H_{15}\right)$ & 108.4 & 110.5 & 107.4 & -- \\
\hline$\alpha\left(C_{11}-C_{12}-N_{16}\right)$ & 111.1 & 111.0 & -- & 110.8 \\
\hline$\alpha\left(C_{11}-C_{12}-C_{19}\right)$ & 108.3 & 111.5 & -- & 111.1 \\
\hline$\alpha\left(\mathrm{H}_{15}-\mathrm{C}_{12}-\mathrm{N}_{16}\right)$ & 112.7 & 107.8 & 107.4 & -- \\
\hline$\alpha\left(H_{15}-C_{12}-C_{19}\right)$ & 106.5 & 109.8 & 107.4 & -- \\
\hline$\alpha\left(N_{16}-C_{12}-C_{19}\right)$ & 109.6 & 106.1 & 111.2 & -- \\
\hline$\alpha\left(C_{12}-N_{16}-H_{17}\right)$ & 111.4 & 112.4 & -- & -- \\
\hline$\alpha\left(C_{12}-N_{16}-H_{18}\right)$ & 111.2 & 113.5 & 107.4 & -- \\
\hline$\alpha\left(C_{12}-N_{16}-H_{24}\right)$ & -- & 102.8 & -- & -- \\
\hline$\alpha\left(\mathrm{H}_{17}-\mathrm{N}_{16}-\mathrm{H}_{18}\right)$ & 107.1 & 107.8 & -- & 108.2 \\
\hline$\alpha\left(\mathrm{H}_{17}-\mathrm{N}_{16}-\mathrm{H}_{24}\right)$ & -- & 108.4 & -- & -- \\
\hline$\alpha\left(\mathrm{H}_{18}-\mathrm{N}_{16}-\mathrm{H}_{24}\right)$ & -- & 111.7 & -- & -- \\
\hline$\alpha\left(C_{12}-C_{19}-O_{20}\right)$ & 122.8 & 116.0 & 125.2 & -- \\
\hline$\alpha\left(C_{12}-C_{19}-O_{21}\right)$ & 114.1 & 114.9 & -- & 117.0 \\
\hline $\mathrm{a}\left(\mathrm{O}_{20}-\mathrm{C}_{19}-\mathrm{O}_{21}\right)$ & 123.1 & 129.1 & -- & 126.4 \\
\hline$\alpha\left(\mathrm{C}_{19}-\mathrm{O}_{21}-\mathrm{H}_{22}\right) / \mathrm{COOH}$ & 105.4 & -- & -- & -- \\
\hline$\alpha\left(C_{1}-O_{22}-H_{23}\right)$ & 109.9 & 110.1 & -- & 111.1 \\
\hline$\delta\left(C_{6}-C_{1}-C_{2}-C_{3}\right)$ & -0.2 & -0.1 & -- & -- \\
\hline$\delta\left(\mathrm{C}_{6}-\mathrm{C}_{1}-\mathrm{C}_{2}-\mathrm{H}_{7}\right)$ & 179.3 & 179.7 & -- & -- \\
\hline$\delta\left(\mathrm{O}_{22}-\mathrm{C}_{1}-\mathrm{C}_{2}-\mathrm{C}_{3}\right)$ & -179.9 & -179.9 & -- & -- \\
\hline$\delta\left(\mathrm{O}_{22}-\mathrm{C}_{1}-\mathrm{C}_{2}-\mathrm{H}_{7}\right)$ & -0.4 & -0.1 & -- & -- \\
\hline$\delta\left(C_{2}-C_{1}-C_{6}-C_{5}\right)$ & 0.3 & 0.1 & -- & -- \\
\hline$\delta\left(\mathrm{C}_{2}-\mathrm{C}_{1}-\mathrm{C}_{6}-\mathrm{H}_{10}\right)$ & -179.3 & -179.6 & -- & -- \\
\hline$\delta\left(\mathrm{O}_{22}-\mathrm{C}_{1}-\mathrm{C}_{6}-\mathrm{C}_{5}\right)$ & -180.0 & -179.9 & -- & -- \\
\hline$\delta\left(\mathrm{O}_{22}-\mathrm{C}_{1}-\mathrm{C}_{6}-\mathrm{H}_{10}\right)$ & 0.5 & 0.2 & -- & -- \\
\hline$\delta\left(\mathrm{C}_{2}-\mathrm{C}_{1}-\mathrm{O}_{22}-\mathrm{H}_{23}\right)$ & 1.1 & 0.8 & -- & -- \\
\hline$\delta\left(\mathrm{C}_{6}-\mathrm{C}_{1}-\mathrm{O}_{22}-\mathrm{H}_{23}\right)$ & -178.6 & -179.0 & -- & -- \\
\hline$\delta\left(C_{1}-C_{2}-C_{3}-C_{4}\right)$ & -0.1 & -0.0 & -- & -- \\
\hline$\delta\left(\mathrm{C}_{1}-\mathrm{C}_{2}-\mathrm{C}_{3}-\mathrm{H}_{8}\right)$ & 178.9 & 179.6 & -- & -- \\
\hline$\delta\left(\mathrm{H}_{7}-\mathrm{C}_{2}-\mathrm{C}_{3}-\mathrm{C}_{4}\right)$ & -179.6 & -179.8 & -- & -- \\
\hline$\delta\left(\mathrm{H}_{7}-\mathrm{C}_{2}-\mathrm{C}_{3}-\mathrm{H}_{8}\right)$ & -0.6 & -0.1 & -- & -- \\
\hline$\delta\left(C_{2}-C_{3}-C_{4}-C_{5}\right)$ & 0.4 & 0.1 & -- & -- \\
\hline$\delta\left(C_{2}-C_{3}-C_{4}-C_{11}\right)$ & -179.1 & -179.6 & -177.8 & -- \\
\hline$\delta\left(\mathrm{H}_{8}-\mathrm{C}_{3}-\mathrm{C}_{4}-\mathrm{C}_{5}\right)$ & -178.6 & -179.5 & -- & -- \\
\hline$\delta\left(\mathrm{H}_{9}-\mathrm{C}_{3}-\mathrm{C}_{4}-\mathrm{C}_{11}\right)$ & 1.9 & 0.8 & -- & -- \\
\hline$\delta\left(C_{3}-C_{4}-C_{5}-C_{6}\right)$ & -0.3 & -0.1 & -- & -- \\
\hline$\delta\left(\mathrm{C}_{3}-\mathrm{C}_{4}-\mathrm{C}_{5}-\mathrm{H}_{9}\right)$ & 179.0 & 179.1 & -- & -- \\
\hline$\delta\left(C_{11}-C_{4}-C_{5}-C_{6}\right)$ & 179.2 & 179.6 & 178.7 & -- \\
\hline$\delta\left(\mathrm{C}_{11}-\mathrm{C}_{4}-\mathrm{C}_{5}-\mathrm{H}_{9}\right)$ & -1.5 & -1.3 & -- & -- \\
\hline$\delta\left(C_{3}-C_{4}-C_{11}-C_{12}\right)$ & 106.8 & 68.8 & -- & -- \\
\hline$\delta\left(C_{3}-C_{4}-C_{11}-H_{13}\right)$ & -14.9 & -50.8 & -- & -- \\
\hline$\delta\left(C_{3}-C_{4}-C_{11}-H_{14}\right)$ & -132.8 & -168.4 & -- & -- \\
\hline
\end{tabular}


Citation: Yadav RA, Dixit V, Yogesh M, Santhosh C (2015) Raman and IR Spectral and DFT Based Vibrational and Electronic Characterization of Isolated and Zwitterionic Forms of L-Tyrosine. Pharm Anal Acta 6: 439. doi:10.4172/21532435.1000439

Page 5 of 18

\begin{tabular}{|c|c|c|c|c|}
\hline$\delta\left(C_{5}-C_{4}-C_{11}-C_{12}\right)$ & -72.7 & -110.8 & -- & -- \\
\hline$\delta\left(\mathrm{C}_{5}-\mathrm{C}_{4}-\mathrm{C}_{11}-\mathrm{H}_{13}\right)$ & 165.6 & 129.5 & -- & -- \\
\hline$\delta\left(\mathrm{C}_{5}-\mathrm{C}_{4}-\mathrm{C}_{11}-\mathrm{H}_{14}\right)$ & 47.8 & 12.0 & -- & -- \\
\hline$\delta\left(C_{4}-C_{5}-C_{6}-C_{1}\right)$ & -0.0 & -0.0 & -- & -- \\
\hline$\delta\left(\mathrm{C}_{4}-\mathrm{C}_{5}-\mathrm{C}_{6}-\mathrm{H}_{10}\right)$ & 179.5 & 179.7 & -- & -- \\
\hline$\delta\left(\mathrm{H}_{9}-\mathrm{C}_{5}-\mathrm{C}_{6}-\mathrm{C}_{1}\right)$ & -179.4 & -179.1 & -- & -- \\
\hline$\delta\left(\mathrm{H}_{9}-\mathrm{C}_{5}-\mathrm{C}_{6}-\mathrm{H}_{10}\right)$ & 0.2 & 0.6 & -- & -- \\
\hline$\delta\left(\mathrm{C}_{4}-\mathrm{C}_{11}-\mathrm{C}_{12}-\mathrm{H}_{15}\right)$ & 60.4 & -60.9 & -- & -- \\
\hline$\delta\left(C_{4}-C_{11}-C_{12}-N_{16}\right)$ & -63.9 & 58.6 & -- & --- \\
\hline$\delta\left(C_{4}-C_{11}-C_{12}-C_{19}\right)$ & 175.6 & 176.7 & 170.36 & -- \\
\hline$\delta\left(\mathrm{H}_{13}-\mathrm{C}_{11}-\mathrm{C}_{12}-\mathrm{H}_{15}\right)$ & -177.2 & 61.3 & -- & -- \\
\hline$\delta\left(\mathrm{H}_{13}-\mathrm{C}_{11}-\mathrm{C}_{12}-\mathrm{N}_{16}\right)$ & 58.4 & -179.1 & -- & -- \\
\hline$\delta\left(\mathrm{H}_{13}-\mathrm{C}_{11}-\mathrm{C}_{12}-\mathrm{C}_{19}\right)$ & -62.1 & -61.1 & -- & -- \\
\hline$\delta\left(\mathrm{H}_{14}-\mathrm{C}_{11}-\mathrm{C}_{12}-\mathrm{H}_{15}\right)$ & -61.9 & 176.0 & -- & -- \\
\hline$\delta\left(\mathrm{H}_{14}-\mathrm{C}_{11}-\mathrm{C}_{12}-\mathrm{N}_{16}\right)$ & 173.7 & -64.4 & -- & -- \\
\hline$\delta\left(\mathrm{H}_{14}-\mathrm{C}_{11}-\mathrm{C}_{12}-\mathrm{C}_{19}\right)$ & 53.2 & 53.6 & -- & -- \\
\hline$\delta\left(\mathrm{C}_{11}-\mathrm{C}_{12}-\mathrm{N}_{16}-\mathrm{H}_{17}\right)$ & 150.2 & -137.0 & -- & -- \\
\hline$\delta\left(\mathrm{C}_{11}-\mathrm{C}_{12}-\mathrm{N}_{16}-\mathrm{H}_{18}\right)$ & 30.9 & -14.3 & -- & -- \\
\hline$\delta\left(\mathrm{C}_{11}-\mathrm{C}_{12}-\mathrm{N}_{16}-\mathrm{H}_{24}\right)$ & -- & 106.5 & -- & -- \\
\hline$\delta\left(\mathrm{H}_{15}-\mathrm{C}_{12}-\mathrm{N}_{16}-\mathrm{H}_{17}\right)$ & 28.4 & -15.9 & -- & -- \\
\hline$\delta\left(\mathrm{H}_{15}-\mathrm{C}_{12}-\mathrm{N}_{16}-\mathrm{H}_{18}\right)$ & -91.0 & 106.9 & -- & -- \\
\hline$\delta\left(\mathrm{H}_{15}-\mathrm{C}_{12}-\mathrm{N}_{16}-\mathrm{H}_{24}\right)$ & -- & -132.3 & -- & -- \\
\hline$\delta\left(\mathrm{C}_{19}-\mathrm{C}_{12}-\mathrm{N}_{16}-\mathrm{H}_{17}\right)$ & -90.1 & 101.7 & -- & -- \\
\hline$\delta\left(\mathrm{C}_{19}-\mathrm{C}_{12}-\mathrm{N}_{16}-\mathrm{H}_{18}\right)$ & 150.5 & -135.5 & -- & -- \\
\hline$\delta\left(\mathrm{C}_{19}-\mathrm{C}_{12}-\mathrm{N}_{16}-\mathrm{H}_{24}\right)$ & -- & -14.7 & -- & -- \\
\hline$\delta\left(\mathrm{C}_{11}-\mathrm{C}_{12}-\mathrm{C}_{19}-\mathrm{O}_{20}\right)$ & -72.8 & 69.2 & -- & --- \\
\hline$\delta\left(C_{11}-C_{12}-C_{19}-O_{21}\right)$ & 105.3 & -109.6 & -- & -- \\
\hline$\delta\left(\mathrm{H}_{15}-\mathrm{C}_{12}-\mathrm{C}_{19}-\mathrm{O}_{20}\right)$ & 43.6 & -53.6 & -- & -- \\
\hline$\delta\left(\mathrm{H}_{15}-\mathrm{C}_{12}-\mathrm{C}_{19}-\mathrm{O}_{21}\right)$ & -138.2 & 127.6 & -- & -- \\
\hline$\delta\left(\mathrm{N}_{16}-\mathrm{C}_{12}-\mathrm{C}_{19}-\mathrm{O}_{20}\right)$ & 165.8 & -169.8 & -- & -- \\
\hline$\delta\left(\mathrm{N}_{16}-\mathrm{C}_{12}-\mathrm{C}_{19}-\mathrm{O}_{21}\right)$ & -16.0 & 11.3 & -- & -- \\
\hline$\delta\left(\mathrm{C}_{12}-\mathrm{C}_{19}-\mathrm{O}_{21}-\mathrm{H}_{22}\right) / \mathrm{COOH}$ & 3.3 & -- & -- & -- \\
\hline$\delta\left(\mathrm{O}_{20}-\mathrm{C}_{19}-\mathrm{O}_{21}-\mathrm{H}_{22}\right)$ & -178.5 & -- & -- & -- \\
\hline
\end{tabular}

The bond lengths and angles are measured in units of $\AA$ and degrees $\left(^{\circ}\right)$ respectively.

Table 3: Geometrical parameters for C-1 conformer of L-TYR.

2.4 degrees respectively for isolated molecule and $0.4,6.1,2.8,2.1$, $0.4,9.2$ and 0.5 degrees respectively in zwitterion. Like bond lengths, one of the bond angles in the $\mathrm{NH}_{3}$ group is exceptionally larger than the others, this is equivalent to saying that the angle $\alpha\left(\mathrm{C}_{12}-\mathrm{N}_{16}-\mathrm{H}_{24}\right)$ is exceptionally shorter than the other two. Overall, the computed and observed geometrical data have been found in good agreement. The discrepancies, if any, could occur due to experimental errors as well as due to the fact that in the observed data collective effect of all the conformers are expected to contribute simultaneously.

\section{Atomic partial charges}

Natural atomic charges: The natural partial atomic charges partially appear on the molecular surface which could predict the entire chemical properties of the molecule [32,33]. Table 4 contains the natural atomic charges at various atomic sites in both the forms of the L-TYR molecule. All the $\mathrm{C}$ atoms, except $\mathrm{C}_{1}$, of the phenyl ring possess negative natural charges having different magnitudes. The $\mathrm{C}_{1}$ and $\mathrm{C}_{4}$ atoms possess the highest and lowest magnitudes of natural charges in phenyl ring. The $\mathrm{C}$ atom of the $\mathrm{COOH} / \mathrm{CO}_{2}$ group possesses the highest positive charge while the other two $\mathrm{C}$ atoms possess negative natural atomic charges in which $\mathrm{C}_{12}$ has smaller value in the side chain for both the considered forms. The magnitude of natural charge at the $\mathrm{N}$ atom is the highest $(-0.88414)$ in isolated molecule while it is sufficiently high (-0.71534) in the zwitterionic form of L-TYR. All the $\mathrm{H}$ atoms in the isolated molecule possess positive natural charges of varying magnitudes while in the zwitterion all the $\mathrm{H}$ atoms, except $\mathrm{H}_{22}$, possess positive natural charges with varying magnitudes. Clearly, the values of natural charges on the $\mathrm{H}$ atoms associated with the ring, directly attached to the side chain and associated with the $\mathrm{OH}$ group have been computed to be $\sim 0.20 \mathrm{e}-0.22 \mathrm{e}, \sim 0.20 \mathrm{e}-0.26 \mathrm{e}$ and $\sim 0.46 \mathrm{e}$ respectively. The two $\mathrm{H}$ atoms of the nitro group possess natural charges $\sim 0.37 \mathrm{e}$ in the isolated molecule while $\sim 0.414 \mathrm{e}$ in the zwitterion; on the other hand $\mathrm{H}_{22}$ in the isolated molecule belonging to the $\mathrm{COOH}$ group possessing very small natural charge $(\sim 0.50 \mathrm{e})$ as compared to that in the zwitterionic form $(\sim 0.67 \mathrm{e})$; where $\mathrm{H}_{22}$ atom is associated with nitro group. It could be noted that in the isolated molecule the $\mathrm{N}$ atom possesses the highest negative natural charge $(\sim-0.88414 \mathrm{e})$ while in the zwitterionic molecule $\mathrm{O}_{21}$ has the highest negative charge $(\sim-0.76072 \mathrm{e})$.

APT atomic charges: The atomic polarizability tensor (APT) charges are expressed as the sum of the charge and charge flux tensors [34]. Table 4 contains the APT atomic charges at various atomic sites of L-TYR. The $\mathrm{C}$ atoms of the ring possess alternately positive and negative ATP charges; both the $\mathrm{C}$ atoms at the juxtra positions of the $\mathrm{C}$ atom directly attached to the side chain, attain much smaller positive APT charge $(0.01 \mathrm{e}-0.04 \mathrm{e})$ than those of the other ring $\mathrm{C}$ atoms $(0.2 \mathrm{e})$. However, the $\mathrm{C}$ atom attached with the $\mathrm{OH}$ group holds highest positive APT charge $(0.613493 \mathrm{e} / 0.812467 \mathrm{e})$ in the ring for isolated / zwitterionic form, while the $\mathrm{C}$ atoms belonging to the side 
Citation: Yadav RA, Dixit V, Yogesh M, Santhosh C (2015) Raman and IR Spectral and DFT Based Vibrational and Electronic Characterization of Isolated and Zwitterionic Forms of L-Tyrosine. Pharm Anal Acta 6: 439. doi:10.4172/21532435.1000439

Page 6 of 18

\begin{tabular}{|c|c|c|c|c|c|c|}
\hline \multirow[t]{2}{*}{ Atom } & \multicolumn{3}{|c|}{ Atomic partial charges (Isolated) } & \multicolumn{3}{|c|}{ Atomic partial charges (Zwitterion) } \\
\hline & APT & Mulliken & Natural & APT & Mulliken & Natural \\
\hline $\mathrm{C}_{1}$ & 0.613493 & -0.435352 & 0.31468 & 0.812467 & -0.470637 & 0.31493 \\
\hline $\mathrm{C}_{2}$ & -0.150654 & -0.047939 & -0.27605 & -0.204435 & -0.028073 & -0.26564 \\
\hline $\mathrm{C}_{3}$ & 0.009604 & -0.358761 & -0.19208 & 0.039308 & -0.398504 & -0.18162 \\
\hline $\mathrm{C}_{4}$ & -0.076057 & 1.151327 & -0.07589 & -0.122508 & 1.317786 & -0.08505 \\
\hline $\mathrm{C}_{5}$ & 0.031030 & -0.179803 & -0.17718 & 0.029461 & -0.277654 & -0.20283 \\
\hline $\mathrm{C}_{6}$ & -0.133771 & -0.206873 & -0.23714 & -0.194348 & -0.269538 & -0.24175 \\
\hline $\mathrm{H}_{7}$ & 0.026331 & 0.147727 & 0.20294 & 0.048736 & 0.195566 & 0.20523 \\
\hline $\mathrm{H}_{8}$ & 0.037415 & 0.175254 & 0.20537 & 0.051569 & 0.200611 & 0.20984 \\
\hline $\mathrm{H}_{9}$ & 0.042596 & 0.178566 & 0.20731 & 0.051611 & 0.203054 & 0.20574 \\
\hline $\mathrm{H}_{10}$ & 0.054919 & 0.201684 & 0.22034 & 0.065244 & 0.215356 & 0.22232 \\
\hline$C_{11}$ & 0.137992 & -0.834036 & -0.39897 & 0.172302 & -0.938486 & -0.41689 \\
\hline$C_{12}$ & 0.139311 & -0.184624 & -0.11510 & 0.173849 & -0.306090 & -0.10537 \\
\hline $\mathrm{H}_{13}$ & -0.016288 & 0.167633 & 0.20843 & 0.000283 & 0.221867 & 0.25676 \\
\hline $\mathrm{H}_{14}$ & 0.001787 & 0.216132 & 0.23650 & -0.023739 & 0.203883 & 0.21498 \\
\hline $\mathrm{H}_{15}$ & -0.028309 & 0.203647 & 0.20346 & 0.006561 & 0.251243 & 0.21008 \\
\hline $\mathrm{N}_{16}$ & -0.518797 & -0.438526 & -0.88414 & -0.488237 & -0.145116 & -0.71534 \\
\hline $\mathrm{H}_{17}$ & 0.153285 & 0.270493 & 0.37001 & 0.272411 & 0.353028 & 0.41455 \\
\hline $\mathrm{H}_{18}$ & 0.167789 & 0.261335 & 0.37780 & 0.310837 & 0.328162 & 0.41415 \\
\hline $\mathrm{C}_{19}$ & 1.156077 & -0.237381 & 0.78978 & 1.677125 & -0.043051 & 0.74312 \\
\hline $\mathrm{O}_{20}$ & -0.777492 & -0.252935 & -0.58933 & -1.227794 & -0.478450 & -0.69520 \\
\hline $\mathrm{O}_{21}$ & -0.789743 & -0.127142 & -0.68590 & -1.282249 & -0.492382 & -0.76072 \\
\hline $\mathrm{H}_{22}$ & 0.397038 & 0.292185 & 0.49854 & -0.994466 & -0.276041 & -0.67002 \\
\hline $\mathrm{O}_{23}$ & -0.766120 & -0.227448 & -0.67111 & 0.363525 & 0.303235 & 0.46933 \\
\hline $\mathrm{H}_{24}$ & 0.288563 & 0.264839 & 0.46772 & 0.462488 & 0.330231 & 0.45942 \\
\hline
\end{tabular}

\# Charges are taken in units of e.

Table 4: APT and Mulliken atomic charges of the lowest energy conformer of L-TYR.

chain possess positive APT charges with different magnitudes. The $\mathrm{C}$ atom of the $\mathrm{COOH} / \mathrm{CO}_{2}$ group possesses the highest APT atomic charge (1.156077e/ 1.677125e), while the other $\mathrm{C}$ sites of the side chain possess $-0.138 \mathrm{e}$ and $-0.173 \mathrm{e}$ APT atomic charges in the isolated and zwitterionic forms. All the $\mathrm{H}$ atoms in the molecule, except $\mathrm{H}_{13}$ and $\mathrm{H}_{15}$ in isolated and $\mathrm{H}_{14}$ and $\mathrm{H}_{22}$ in zwitterion, possess positive APT charges with varying magnitudes. Clearly, $\mathrm{H}_{14}$ in isolated and $\mathrm{H}_{13}$ in zwitterion possess the smallest magnitude of APT charges $(0.001787 \mathrm{e} / 0.000283 \mathrm{e})$ while in the two considered species of the molecule amongst all the $\mathrm{H}$ atoms, $\mathrm{H}_{22}$ possesses the highest magnitude of APT charge (0.397038/ $-0.994466 \mathrm{e})$. The $\mathrm{N}$ and the three $\mathrm{O}$ atoms possess negative APT charges with the magnitudes $-0.518797 \mathrm{e} /-0.488237 \mathrm{e},-0.777492 \mathrm{e} /-1.227794 \mathrm{e}$, $-0.789743 \mathrm{e} /-1.282249 \mathrm{e}$ and $-0.766120 \mathrm{e} / 0.363525 \mathrm{e}$ in the isolated/ zwitterionic forms of L-TYR. It is noticeable that the APT charges at the $\mathrm{O}$ atoms in the $\mathrm{CO}_{2}$ group are nearly twice the APT charges at the $\mathrm{O}$ atoms in the $\mathrm{COOH}$ group while the APT charge at the $\mathrm{O}$ atom of the $\mathrm{OH}$ group in the isolated molecule has been found to be twice that at the corresponding site in the zwitterionic molecule. The APT charge at $\mathrm{H}_{22}(0.397038 \mathrm{e})$ in isolated becomes $-0.994466 \mathrm{e}$ in the zwitterion state. Conclusively, the APT charges are found to vary to large extent at every reactive site when molecule goes from the isolated state to the zwitterionic state.

Mulliken atomic charges: The Mulliken atomic charge calculation plays a crucial role in the application of quantum chemical calculation to the molecular systems because these charges are responsible for the dipole moment, polarizability, electronic structure, and much more characteristics of the molecular systems. The Mulliken atomic charges at all the sites of the most stable conformers of the two species of the L-TYR molecule have been collected in the Table-4, from which it is interesting to notice that all the $\mathrm{C}$ atoms as well as the highly electronegative atoms, namely, $\mathrm{O}$ and $\mathrm{N}$ atoms in the molecule, except $\mathrm{C}_{4}$ in both and $\mathrm{O}_{23}$ in zwitterion, possess negative Mulliken atomic charges with different magnitudes. The largest and smallest values of the Mulliken atomic charge corresponding to the $\mathrm{C}$ atoms in the optimized structure are possessed by $\mathrm{C}_{4} 1.151327 \mathrm{e} / 1.317786 \mathrm{e}$ and $\mathrm{C}_{2}-0.047939 \mathrm{e} / \mathrm{-}-0.028073 \mathrm{e}$ in the isolated/ zwitterionic species of the L-TYR. The Mulliken atomic charges for all the $\mathrm{H}$ atoms in the molecule, except $\mathrm{H}_{22}(-0.276041 \mathrm{e})$ in the zwitterion, are positive with different values. The $\mathrm{H}$ atoms associated with the ring, attached tetrahedrally to $\mathrm{C}$ atoms and pyramidally to $\mathrm{N}$ atom, belonging to the $\mathrm{COOH}$ group and associated with the $\mathrm{OH}$ group possess the Mulliken atomic charges $0.15 \mathrm{e}$ to $0.20 \mathrm{e} / 0.20 \mathrm{e}$ to $0.22 \mathrm{e}, 0.17 \mathrm{e}$ to $0.20 \mathrm{e} / 0.22 \mathrm{e}$ to $0.25 \mathrm{e}, 0.26 \mathrm{e}$ to $0.27 \mathrm{e} / 0.33 \mathrm{e}$ to $0.35 \mathrm{e}$ and $0.29 \mathrm{e}, 0.26 \mathrm{e} / 0.33 \mathrm{e}$ respectively in the isolated/ zwitterionic forms of the molecule. However, $\mathrm{H}_{22}$ in the zwitterionic molecule possesses exceptionally high value of Mulliken atomic charge $(-0.276041 \mathrm{e})$. It is noticeable that the values of the Mulliken charges at different sites in zwitterionic form appear to be higher than those at the corresponding sites in the isolated molecule. The electronegative atoms $\mathrm{N}_{16}, \mathrm{O}_{20}, \mathrm{O}_{21}$ and $\mathrm{O}_{23}$ possess Mulliken charges $-0.438526 \mathrm{e} /-0.145116 \mathrm{e},-0.252935 \mathrm{e} /-0.478450 \mathrm{e},-0.127142 \mathrm{e} /$ $-0.492382 \mathrm{e}$ and $-0.227448 \mathrm{e} /+0.330231 \mathrm{e}$ respectively in the isolated and zwitterionic states of L-TYR.

\section{Vibrational assignments}

The L-TYR molecule, consisting of 24 atoms, has 66 normal modes of vibration as detailed in Table 5 . The calculated and observed vibrational frequencies along with the corresponding PEDs with proposed vibrational assignments have been collected in Table 6 (isolated molecule) and Table 7 (zwitterion). In Figures 2-5 the experimentally observed and computed IR and Raman spectra in different frequency ranges have been compared.

In the experimental IR spectrum a weak peak is observed at $~ 2075$ 
Citation: Yadav RA, Dixit V, Yogesh M, Santhosh C (2015) Raman and IR Spectral and DFT Based Vibrational and Electronic Characterization of Isolated and Zwitterionic Forms of L-Tyrosine. Pharm Anal Acta 6: 439. doi:10.4172/21532435.1000439

Page 7 of 18

\begin{tabular}{|c|c|c|c|c|c|}
\hline S.N. & Modes & Total No. & Symbol & Groups & $\begin{array}{l}\text { No. of Modes in Zw/ Iso. } \\
\text { Forms) }\end{array}$ \\
\hline 1 & Stretching & 24 & $\mathrm{v}$ & $\begin{array}{c}\mathrm{C}-\mathrm{C} \\
\mathrm{C}-\mathrm{H} \\
\mathrm{C}-\mathrm{OH} \\
\mathrm{O}-\mathrm{H} \\
\mathrm{CH}_{2} \\
\mathrm{~N}-\mathrm{H}, \mathrm{NH}_{2} \\
\mathrm{CO}_{2} / \mathrm{C}=\mathrm{O} \\
\mathrm{C}-\mathrm{N}\end{array}$ & $\begin{array}{c}9 \\
5 \\
1 / 2 \\
1 / 2 \\
2 \\
3 / 2 \\
2 / 1 \\
1\end{array}$ \\
\hline 2 & In plane bending ${ }^{@}$ & 6 & $\beta$ & $\begin{array}{l}\mathrm{C}-\mathrm{H} \\
\mathrm{C}-\mathrm{O} \\
\mathrm{C}_{4}-\mathrm{C}_{12}\end{array}$ & $\begin{array}{l}4 \\
1 \\
1\end{array}$ \\
\hline 3 & Out of plane bending ${ }^{@}$ & 6 & $Y$ & $\begin{array}{l}\mathrm{C}-\mathrm{H} \\
\mathrm{C}-\mathrm{O} \\
\mathrm{C}_{4}-\mathrm{C}_{12}\end{array}$ & $\begin{array}{l}4 \\
1 \\
1\end{array}$ \\
\hline 4 & Twisting & $6 / 7$ & $T$ & $\begin{array}{c}\mathrm{C}-\mathrm{C} \\
\mathrm{C}-\mathrm{OH} \\
\mathrm{NH}_{3} / \mathrm{NH}_{2} \\
\mathrm{CH}_{2}\end{array}$ & $\begin{array}{c}3 \\
1 / 2 \\
1 \\
1\end{array}$ \\
\hline 5 & Rocking & $4 / 3$ & $\rho, \rho$ & $\begin{array}{c}\mathrm{NH}_{3} / \mathrm{NH}_{2} \\
\stackrel{\mathrm{CH}}{\mathrm{H}_{2}} \\
\mathrm{CO}_{2} / \mathrm{COOH}\end{array}$ & $\begin{array}{c}2 / 1 \\
1 \\
1\end{array}$ \\
\hline 6 & Wagging & $2 / 3$ & $\omega$ & $\begin{array}{c}\mathrm{CH}_{2} \\
\mathrm{CO}_{2} / \mathrm{COOH} \\
\mathrm{NH}_{3} / \mathrm{NH}_{2}\end{array}$ & $\begin{array}{c}1 \\
1 \\
0 / 1\end{array}$ \\
\hline 7 & Scissoring & $2 / 3$ & $\delta$ & $\begin{array}{c}\mathrm{CH}_{2} \\
\mathrm{CO}_{2} / \mathrm{COOH} \\
\mathrm{NH}_{3} / \mathrm{NH}_{2}\end{array}$ & $\begin{array}{c}1 \\
1 \\
0 / 1\end{array}$ \\
\hline 8 & Angle bending & $7 / 8$ & $\alpha$ & $\begin{array}{l}\mathrm{C}-\mathrm{C}-\mathrm{C} \\
\mathrm{C}-\mathrm{C}-\mathrm{N} \\
\mathrm{H}-\mathrm{C}-\mathrm{N} \\
\mathrm{C}-\mathrm{O}-\mathrm{H}\end{array}$ & $\begin{array}{c}2 \\
2 \\
2 \\
1 / 2\end{array}$ \\
\hline 9 & Deformation & $3 / 0$ & $\delta, \delta$ & $\mathrm{NH}_{3} / \mathrm{NH}_{2}$ & $3 / 0$ \\
\hline 10 & Ring planar deformation & 3 & $\alpha$ & $\alpha(R)$ & 3 \\
\hline 11 & Ring non-planar deformation & 3 & $\Phi$ & $\Phi(\mathrm{R})$ & 3 \\
\hline
\end{tabular}

Table 5: Normal modes distribution in the most stable conformers of isolated/ zwitterionic form of L-TYR.

\begin{tabular}{|c|c|c|c|c|c|c|}
\hline \multirow[t]{2}{*}{ Mode } & \multicolumn{2}{|c|}{ Computed Frequencies } & \multicolumn{2}{|c|}{ Observed Freq. } & \multirow[t]{2}{*}{ PEDs } & \multirow[t]{2}{*}{ Mode Assignment } \\
\hline & Unscaled & Scaled & Raman & IR & & \\
\hline$v_{1}$ & $3835(75,134) 0.23$ & 3662 & - & $3430^{\#}$ & $\mathrm{v}\left(\mathrm{O}_{23}-\mathrm{H}_{24}\right)(100)$ & $\mathrm{v}\left(\mathrm{O}_{23}-\mathrm{H}_{24}\right)$ \\
\hline$v_{2}$ & $3576(24,51) 0.75$ & 3415 & - & - & $\mathrm{v}_{\text {asym }}\left(\mathrm{NH}_{2}\right)(100)$ & $\mathrm{v}_{\text {asym }}\left(\mathrm{NH}_{2}\right)$ \\
\hline$v_{3}$ & $3507(12,94) 0.13$ & 3349 & - & - & $\mathrm{v}_{\text {sym }}\left(\mathrm{NH}_{2}\right)(90)-\mathrm{v}\left(\mathrm{O}_{21}-\mathrm{H}_{22}\right)(10)$ & $\mathrm{v}_{\text {sym }}\left(\mathrm{NH}_{2}\right)$ \\
\hline$v_{4}$ & $3481(251,115) 0.07$ & 3324 & $3207^{\#}$ & 3207 & $\mathrm{v}\left(\mathrm{O}_{21}-\mathrm{H}_{22}\right)(89)+\mathrm{v}\left(\mathrm{N}_{16}-\mathrm{H}_{18}\right)(7)$ & $\mathrm{v}\left(\mathrm{O}_{21}-\mathrm{H}_{22}\right)$ \\
\hline$v_{5}$ & $3194(4,144) 0.22$ & 3050 & $3061^{\#}$ & $\begin{array}{l}3041 \\
3068^{\#}\end{array}$ & $\mathrm{v}\left(\mathrm{C}_{6}-\mathrm{H}_{10}\right)(94)$ & $\mathrm{v}(\mathrm{CH})$ \\
\hline$v_{6}$ & $3166(9,135) 0.16$ & 3024 & $3042^{\#}$ & $\begin{array}{l}3025, \\
3042^{\#}\end{array}$ & $v\left(C_{3}-H_{8}\right)(65)+v\left(C_{2}-H_{7}\right)(33)$ & $\mathrm{v}(\mathrm{CH})$ \\
\hline$v_{7}$ & $3160(8,48) 0.51$ & 3018 & $3027^{\#}$ & $3024^{\#}$ & $\mathrm{v}\left(\mathrm{C}_{5}-\mathrm{H}_{9}\right)(92)$ & $\mathrm{v}(\mathrm{CH})$ \\
\hline$v_{8}$ & $3149(16,64) 0.61$ & 3007 & $3014^{\#}$ & $3014^{\#}$ & $\mathrm{v}\left(\mathrm{C}_{2}-\mathrm{H}_{7}\right)(66)-\mathrm{v}\left(\mathrm{C}_{3}-\mathrm{H}_{8}\right)(33)$ & $\mathrm{v}(\mathrm{CH})$ \\
\hline$v_{9}$ & $3090(6,50) 0.41$ & 2951 & $2950^{\#}$ & $\begin{array}{l}2960 \\
2962^{\#}\end{array}$ & $\mathrm{v}_{\text {asym }}\left(\mathrm{CH}_{2}\right)(98)$ & $\mathrm{v}_{\text {asym }}\left(\mathrm{CH}_{2}\right)$ \\
\hline$v_{10}$ & $3043(23,25) 0.21$ & 2906 & $2923^{\#}$ & $\begin{array}{l}2931 \\
2928^{\#}\end{array}$ & $\mathrm{v}_{\text {sym }}\left(\mathrm{CH}_{2}\right)(60)-\mathrm{v}\left(\mathrm{C}_{12}-\mathrm{H}_{15}\right)(39)$ & $\mathrm{v}_{\text {sym }}\left(\mathrm{CH}_{2}\right)$ \\
\hline $\mathrm{v}_{11}$ & $3032(5,116) 0.10$ & 2896 & $2863^{\#}$ & $\begin{array}{l}2894 \\
2864^{\#}\end{array}$ & $\mathrm{v}\left(\mathrm{C}_{12}-\mathrm{H}_{15}\right)(59)+\mathrm{v}_{\text {sym }}\left(\mathrm{CH}_{2}\right)(40)$ & $\mathrm{v}\left(\mathrm{C}_{12}-\mathrm{H}_{15}\right)$ \\
\hline$v_{12}$ & $1835(374,15) 0.27$ & 1752 & - & 1630\# & $\mathrm{v}\left(\mathrm{C}_{19}-\mathrm{O}_{20}\right)(78)$ & $\mathrm{v}\left(\mathrm{C}_{19}-\mathrm{O}_{20}\right)$ \\
\hline$v_{13}$ & $1663(29,5) 0.74$ & 1588 & $1587,1590^{\#}$ & $\begin{array}{l}1582 \\
1589^{\#}\end{array}$ & $\delta\left(\mathrm{NH}_{2}\right)(95)$ & $\delta\left(\mathrm{NH}_{2}\right)$ \\
\hline $\mathrm{v}_{14}$ & $1653(49,56) 0.52$ & 1579 & 1567 & 1579 & $\begin{array}{c}v\left(C_{5}-C_{6}\right)(20)+v\left(C_{2}-C_{3}\right)(18)-v\left(C_{1}-C_{6}\right)(10)-v\left(C_{3}-C_{4}\right) \\
(10)+\alpha_{2}(R)(11)+\beta\left(C_{3}-H_{8}\right)(6)\end{array}$ & $v(R)$ \\
\hline $\mathrm{v}_{15}$ & $1629(15,5) 0.72$ & 1556 & $1551,1531^{\#}$ & $\begin{array}{l}1572 \\
1532^{\#}\end{array}$ & $\begin{array}{c}v\left(C_{1}-C_{2}\right)(24)+v\left(C_{4}-C_{5}\right)(17)-v\left(C_{1}-C_{6}\right)(16)-v\left(C_{3}-C_{4}\right) \\
(10)-a_{3}(R)(8)\end{array}$ & $v(R)$ \\
\hline$v_{16}$ & $1543(105,2) 0.70$ & 1474 & $1481,1463^{\#}$ & $\begin{array}{l}1453 \\
1454^{\#}\end{array}$ & $\begin{array}{c}v\left(C_{1}-C_{6}\right)(10)-v\left(C_{3}-C_{4}\right)(10)+v\left(C_{1}-C_{2}\right)(9)-v\left(C_{4}-C_{5}\right) \\
(7)-\beta\left(C_{6}-H_{10}\right)(10)+\beta\left(C_{2}-H_{7}\right)(14)+\beta\left(C_{3}-H_{8}\right) \\
(12)-\beta\left(C_{5}-H_{9}\right)(13)-v\left(C_{1}-O_{23}\right)(8)\end{array}$ & $\beta(\mathrm{CH})$ \\
\hline $\mathrm{v}_{17}$ & $1489(6,6) 0.73$ & 1422 & $1419,1414^{\#}$ & $\begin{array}{l}1435 \\
1438^{\#}\end{array}$ & $\delta\left(\mathrm{CH}_{2}\right)(89)$ & $\delta\left(\mathrm{CH}_{2}\right)$ \\
\hline
\end{tabular}


Citation: Yadav RA, Dixit V, Yogesh M, Santhosh C (2015) Raman and IR Spectral and DFT Based Vibrational and Electronic Characterization of Isolated and Zwitterionic Forms of L-Tyrosine. Pharm Anal Acta 6: 439. doi:10.4172/21532435.1000439

Page 8 of 18

\begin{tabular}{|c|c|c|c|c|c|c|}
\hline $\mathrm{v}_{18}$ & $1466(13,0) 0.75$ & 1400 & - & $\begin{array}{l}1417, \\
1416^{\#}\end{array}$ & $\begin{array}{c}v\left(C_{5}-C_{6}\right)(17)+v\left(C_{2}-C_{3}\right)(16)+v\left(C_{4}-C_{5}\right)(6)+\beta\left(C_{6}-H_{10}\right) \\
(12)+\beta\left(C_{2}-H_{7}\right)(9)-\beta\left(C_{3}-H_{8}\right)(7)-\alpha\left(C_{1}-O_{23}-H_{24}\right)(8)\end{array}$ & $v(R)$ \\
\hline $\mathrm{v}_{19}$ & $1412(433,6) 0.61$ & 1348 & $1365,1368^{\#}$ & $\begin{array}{l}1363 \\
1367^{\#}\end{array}$ & $\mathrm{a}\left(\mathrm{C}_{19}-\mathrm{O}_{21}-\mathrm{H}_{22}\right)(65)-\mathrm{v}\left(\mathrm{C}_{1}-\mathrm{C}_{6}\right)(15)$ & $\alpha\left(C_{19}-O_{21}-H_{22}\right)$ \\
\hline$v_{20}$ & $1376(14,21) 0.40$ & 1314 & $1327,1328^{\#}$ & $\begin{array}{l}1332 \\
1328^{\#}\end{array}$ & $\begin{array}{c}\alpha\left(\mathrm{C}_{11}-\mathrm{C}_{12}-\mathrm{H}_{15}\right)(23)-\omega\left(\mathrm{CH}_{2}\right)(23)-\rho\left(\mathrm{CH}_{2}\right)(11)- \\
v\left(\mathrm{C}_{11}-\mathrm{C}_{12}\right)(6)\end{array}$ & $\mathrm{a}\left(\mathrm{C}_{11}-\mathrm{C}_{12}-\mathrm{H}_{15}\right)$ \\
\hline $\mathrm{v}_{21}$ & $1361(30,1) 0.58$ & 1300 & - & - & $\begin{array}{c}\beta\left(\mathrm{C}_{5}-\mathrm{H}_{9}\right)(11)+\beta\left(\mathrm{C}_{3}-\mathrm{H}_{8}\right)(10)-\alpha\left(\mathrm{C}_{1}-\mathrm{O}_{23}-\mathrm{H}_{24}\right)(11)-\alpha\left(\mathrm{N}_{16}-\right. \\
\left.\mathrm{C}_{12}-\mathrm{H}_{15}\right)(10)+v\left(\mathrm{C}_{2}-\mathrm{C}_{3}\right)(9)-v\left(\mathrm{C}_{1}-\mathrm{C}_{2}\right)(8)-\mathrm{v}\left(\mathrm{C}_{5}-\mathrm{C}_{6}\right) \\
(7)-v\left(\mathrm{C}_{3}-\mathrm{C}_{4}\right)(6) v\left(\mathrm{C}_{3}-\mathrm{C}_{4}\right)(6)+v\left(\mathrm{C}_{1}-\mathrm{C}_{6}\right)(6)+\omega\left(\mathrm{CH}_{2}\right)(6)\end{array}$ & $\beta(\mathrm{CH})$ \\
\hline$v_{22}$ & $1358(19,9) 0.32$ & 1297 & - & $1286^{\#}$ & $\alpha\left(\mathrm{N}_{16}-\mathrm{C}_{12}-\mathrm{H}_{15}\right)(27)-\omega\left(\mathrm{CH}_{2}\right)(25)+\beta\left(\mathrm{C}_{5}-\mathrm{H}_{9}\right)(7)$ & $\mathrm{a}\left(\mathrm{N}_{16}-\mathrm{C}_{12}-\mathrm{H}_{15}\right)$ \\
\hline $\mathrm{v}_{23}$ & $1344(3,4) 0.46$ & 1284 & $1284,1286^{\#}$ & 1267 & $\begin{array}{c}\alpha\left(C_{11}-C_{12}-H_{15}\right)(14)+v\left(C_{3}-C_{4}\right)(9)-v\left(C_{4}-C_{5}\right)(8)+v\left(C_{5}-C_{6}\right) \\
(5)+\beta\left(C_{2}-H_{7}\right)(8)+\beta\left(C_{6}-H_{10}\right)(8)+\alpha\left(N_{16}-C_{12}-H_{15}\right)(8)+ \\
\rho\left(C_{2}\right)(6)\end{array}$ & $\mathrm{v}(\mathrm{R})$ \\
\hline $\mathrm{v}_{24}$ & $1292(4,1) 0.67$ & 1234 & - & $\begin{array}{l}1247 \\
1246^{\#}\end{array}$ & $\begin{array}{c}\omega\left(\mathrm{CH}_{2}\right)(22)+\rho\left(\mathrm{NH}_{2}\right)(21)+\alpha\left(\mathrm{N}_{16}-\mathrm{C}_{12}-\mathrm{H}_{15}\right)(12)+ \\
\alpha\left(\mathrm{C}_{11}-\mathrm{C}_{12}-\mathrm{H}_{15}\right)(11)\end{array}$ & $\omega\left(\mathrm{CH}_{2}\right)$ \\
\hline $\mathrm{v}_{25}$ & $1288(4,14) 0.25$ & 1230 & $1249,1247^{\#}$ & 1243 & $\begin{array}{c}\alpha\left(\mathrm{N}_{16}-\mathrm{C}_{12}-\mathrm{H}_{15}\right)(24)-\rho\left(\mathrm{CH}_{2}\right)(16)+\alpha\left(\mathrm{C}_{11}-\mathrm{C}_{12}-\mathrm{H}_{15}\right)(14)- \\
\rho\left(\mathrm{NH}_{2}\right)(11)\end{array}$ & $\rho\left(\mathrm{CH}_{2}\right)$ \\
\hline$v_{26}$ & $1280(115,12) 0.03$ & 1222 & $1214,1212^{\#}$ & $\begin{array}{l}1214 \\
1213^{\#}\end{array}$ & $v\left(C_{1}-O_{23}\right)(47)-\alpha_{1}(R)(9)-v\left(C_{2}-C_{3}\right)(8)+\beta\left(C_{2}-H_{7}\right)(8)$ & $\mathrm{v}\left(\mathrm{C}_{1}-\mathrm{O}_{23}\right)$ \\
\hline $\mathrm{v}_{27}$ & $1225(2,27) 0.09$ & 1170 & $1164,1178^{\#}$ & $\begin{array}{l}1176 \\
1177^{\#}\end{array}$ & $\begin{array}{c}v\left(C_{4}-C_{11}\right)(39)-a_{1}(R)(13)-v\left(C_{2}-C_{3}\right)(6)-v\left(C_{3}-C_{4}\right) \\
(6)-v\left(C_{4}-C_{5}\right)(6)\end{array}$ & $v(R)$ \\
\hline $\mathrm{v}_{28}$ & $1210(18,7) 0.73$ & 1156 & $1156,1150^{\#}$ & $\begin{array}{l}1155 \\
1153^{\#}\end{array}$ & $\begin{array}{c}\mathrm{v}\left(\mathrm{C}_{19}-\mathrm{O}_{21}\right)(33)-\mathrm{v}\left(\mathrm{C}_{12}-\mathrm{C}_{19}\right)(16)+\rho\left(\mathrm{CH}_{2}\right)(13)+\alpha\left(\mathrm{C}_{19}-\right. \\
\left.\mathrm{O}_{21}-\mathrm{H}_{22}\right)(8)-\delta(\mathrm{COOH})(6)+\rho(\mathrm{COOH})(6)+\omega\left(\mathrm{NH}_{2}\right)(6)\end{array}$ & $v\left(\mathrm{C}_{19}-\mathrm{O}_{21}\right)$ \\
\hline $\mathrm{v}_{29}$ & $1195(3,4) 0.31$ & 1141 & - & - & $\begin{array}{c}\beta\left(C_{2}-H_{7}\right)(26)-\beta\left(C_{3}-H_{8}\right)(18)-\beta\left(C_{6}-H_{10}\right)(17)-\beta\left(C_{5}-H_{9}\right) \\
(16)+v\left(C_{2}-C_{3}\right)(7)\end{array}$ & $\beta(\mathrm{CH})$ \\
\hline $\mathrm{v}_{30}$ & $1187(207,12) 0.11$ & 1134 & $1114,1113^{\#}$ & $\begin{array}{l}1113 \\
1112^{\#}\end{array}$ & $\begin{array}{c}\alpha\left(C_{1}-O_{23}-H_{24}\right)(48)+v\left(C_{1}-C_{6}\right)(15)+\beta\left(C_{6}-H_{10}\right)(8)- \\
v\left(C_{1}-O_{23}\right)(7)-v\left(C_{1}-C_{2}\right)(6)\end{array}$ & $\alpha(\mathrm{C}-\mathrm{OH}) / \mathrm{OH}$ \\
\hline $\mathrm{v}_{31}$ & $1160(10,3) 0.44$ & 1108 & $1100,1098^{\#}$ & - & $\begin{array}{c}\rho\left(\mathrm{NH}_{2}\right)(19)-\rho\left(\mathrm{CH}_{2}\right)(18)-\alpha\left(\mathrm{C}_{11}-\mathrm{C}_{12}-\mathrm{H}_{15}\right)(17)+ \\
v\left(\mathrm{C}_{19}-\mathrm{O}_{21}\right)(6)\end{array}$ & $\rho\left(\mathrm{NH}_{2}\right)$ \\
\hline $\mathrm{v}_{32}$ & $1120(20,1) 0.66$ & 1070 & - & $\begin{array}{l}1099 \\
1099^{\#}\end{array}$ & $\begin{array}{c}\beta\left(C_{6}-H_{10}\right)(12)-\beta\left(C_{5}-H_{9}\right)(10)+\beta\left(C_{2}-H_{7}\right)(9)-\beta\left(C_{3}-H_{8}\right) \\
(7)+v\left(C_{2}-C_{3}\right)(10)-v\left(C_{5}-C_{6}\right)(9)+v\left(C_{12}-N_{16}\right)(8)\end{array}$ & $\beta(\mathrm{CH})$ \\
\hline $\mathrm{v}_{33}$ & $1097(14,12) 0.54$ & 1048 & $1045,1046^{\#}$ & $\begin{array}{l}1042 \\
1046^{\#}\end{array}$ & $v\left(C_{12}-N_{16}\right)(39)-v\left(C_{11}-C_{12}\right)(15)+\rho\left(C_{2}\right)(7)-\beta\left(C_{12}-C_{19}\right)$ & $v\left(C_{11}-C_{12}\right)$ \\
\hline $\mathrm{v}_{34}$ & $1029(0,0) 0.04$ & 983 & - & - & $\begin{array}{c}\alpha_{1}(R)(49)+v\left(C_{1}-C_{6}\right)(11)+v\left(C_{1}-C_{2}\right)(10)-v\left(C_{3}-C_{4}\right) \\
(7)-v\left(C_{4}-C_{5}\right)(7)\end{array}$ & $\alpha_{1}(R)$ \\
\hline $\mathrm{v}_{35}$ & $979(48,6) 0.29$ & 958 & $986,985^{\#}$ & $986,988^{\#}$ & $\begin{array}{c}T\left(\mathrm{CH}_{2}\right)(15)-\gamma\left(\mathrm{C}_{5}-\mathrm{H}_{9}\right)(14)+\omega\left(\mathrm{NH}_{2}\right)(10)+\gamma\left(\mathrm{C}_{6}-\mathrm{H}_{10}\right) \\
(8)-\rho\left(\mathrm{NH}_{2}\right)(6)-\omega\left(\mathrm{NH}_{2}\right)(6)\end{array}$ & $\mathrm{T}\left(\mathrm{CH}_{2}\right)$ \\
\hline$v_{36}$ & $973(15,2) 0.35$ & 952 & $967^{\#}$ & $939,939^{\#}$ & $Y\left(\mathrm{C}_{5}-\mathrm{H}_{9}\right)(37)-\mathrm{V}\left(\mathrm{C}_{6}-\mathrm{H}_{10}\right)(26)+\mathrm{T}\left(\mathrm{CH}_{2}\right)(6)$ & $\mathrm{Y}(\mathrm{CH})$ \\
\hline$v_{37}$ & $948(48,2) 0.57$ & 928 & $935,938^{\#}$ & $929^{\#}$ & $\begin{array}{c}\omega\left(\mathrm{NH}_{2}\right)(24)-v\left(\mathrm{C}_{11}-\mathrm{C}_{12}\right)(23)+\mathrm{v}\left(\mathrm{C}_{3}-\mathrm{H}_{8}\right)(14)+ \\
v\left(\mathrm{C}_{12}-\mathrm{C}_{19}\right)(7)\end{array}$ & $\mathrm{v}\left(\mathrm{C}_{12}-\mathrm{C}_{19}\right)$ \\
\hline$v_{38}$ & $942(7,1) 0.74$ & 922 & $894,897^{\#}$ & - & $\mathrm{Y}\left(\mathrm{C}_{3}-\mathrm{H}_{8}\right)(43)-\mathrm{V}\left(\mathrm{C}_{2}-\mathrm{H}_{7}\right)(16)-\Phi_{1}(\mathrm{R})(13)$ & $\mathrm{Y}(\mathrm{CH})$ \\
\hline $\mathrm{v}_{39}$ & $887(22,16) 0.02$ & 868 & 878 & $896,899^{\#}$ & $\begin{array}{c}\gamma\left(\mathrm{C}_{6}-\mathrm{H}_{10}\right)(10)+\alpha\left(\mathrm{C}_{4}-\mathrm{C}_{11}-\mathrm{C}_{12}\right)(10)+\omega\left(\mathrm{NH}_{2}\right)(10)+ \\
\gamma\left(\mathrm{C}_{2}-\mathrm{H}_{7}\right)(9)+\omega(\mathrm{COOH})(7)-\mathrm{V}\left(\mathrm{C}_{4}-\mathrm{C}_{11}\right)(6)\end{array}$ & $\omega\left(\mathrm{NH}_{2}\right)$ \\
\hline $\mathrm{v}_{40}$ & $874(46,1) 0.52$ & 855 & $855^{\#}$ & $878,878^{\#}$ & $\begin{array}{c}\mathrm{T}\left(\mathrm{C}_{19}-\mathrm{O}_{21}\right)(33)+\mathrm{T}\left(\mathrm{CH}_{2}\right)(13)-\mathrm{v}\left(\mathrm{C}_{12}-\mathrm{N}_{16}\right)(13)-\omega\left(\mathrm{NH}_{2}\right) \\
(10)-\mathrm{v}\left(\mathrm{C}_{11}-\mathrm{C}_{12}\right)(8)\end{array}$ & $\mathrm{v}\left(\mathrm{C}_{12}-\mathrm{N}_{16}\right)$ \\
\hline $\mathrm{v}_{41}$ & $855(51,12) 0.14$ & 837 & $845,846^{\#}$ & $842,842^{\#}$ & $\begin{array}{c}\mathrm{T}\left(\mathrm{C}_{19}-\mathrm{O}_{21}\right)(36)+\omega\left(\mathrm{NH}_{2}\right)(9)-\mathrm{T}\left(\mathrm{CH}_{2}\right)(7)-\alpha_{2}(\mathrm{R})(7)+ \\
v\left(\mathrm{C}_{12}-\mathrm{N}_{16}\right)(6)\end{array}$ & $\mathrm{T}\left(\mathrm{C}_{19}-\mathrm{O}_{21}\right)$ \\
\hline $\mathrm{v}_{42}$ & $846(20,11) 0.04$ & 828 & $828,829^{\#}$ & $829,830^{\#}$ & $\begin{array}{c}\omega\left(\mathrm{NH}_{2}\right)(21)+\mathrm{T}\left(\mathrm{C}_{19}-\mathrm{O}_{21}\right)(13)-\mathrm{v}\left(\mathrm{C}_{12}-\mathrm{C}_{19}\right)(10)+\mathrm{a}_{3}(\mathrm{R}) \\
(8)+\mathrm{v}\left(\mathrm{C}_{1}-\mathrm{O}_{23}\right)(7)+\mathrm{v}\left(\mathrm{C}_{1}-\mathrm{C}_{6}\right)(6)\end{array}$ & $v(R)$ \\
\hline $\mathrm{v}_{43}$ & $837(29,2) 0.09$ & 819 & 808 & $803,808^{\#}$ & $\gamma\left(\mathrm{C}_{6}-\mathrm{H}_{10}\right)(40)+\gamma\left(\mathrm{C}_{5}-\mathrm{H}_{9}\right)(28)-\mathrm{Y}\left(\mathrm{C}_{1}-\mathrm{O}_{23}\right)(8)$ & $\mathrm{Y}(\mathrm{CH})$ \\
\hline $\mathrm{v}_{44}$ & $815(25,1) 0.17$ & 798 & $796,797^{\#}$ & $793,799^{\#}$ & $\mathrm{~V}\left(\mathrm{C}_{2}-\mathrm{H}_{7}\right)(56)+\mathrm{v}\left(\mathrm{C}_{3}-\mathrm{H}_{8}\right)(22)-\mathrm{v}\left(\mathrm{C}_{1}-\mathrm{O}_{23}\right)(7)$ & $\mathrm{Y}(\mathrm{CH})$ \\
\hline $\mathrm{v}_{45}$ & $792(14,8) 0.09$ & 775 & $741,738^{\#}$ & $741,739^{\#}$ & $\begin{array}{l}\alpha_{1}(R)(17)-v\left(C_{12}-C_{19}\right)(12)-v\left(C_{1}-O_{23}\right)(10)-\delta(C O O H) \\
(10)+v\left(C_{4}-C_{11}\right)(9)+\omega(C O O H)(8)-v\left(C_{19}-O_{21}\right)(6)\end{array}$ & $\omega(\mathrm{COOH})$ \\
\hline $\mathrm{v}_{46}$ & $730(2,2) 0.72$ & 714 & - & - & $\Phi_{1}(\mathrm{R})(60)+\gamma\left(\mathrm{C}_{4}-\mathrm{C}_{11}\right)(12)-\mathrm{\gamma}\left(\mathrm{C}_{1}-\mathrm{O}_{23}\right)(12)-\omega(\mathrm{COOH})$ & $\Phi_{1}(\mathrm{R})$ \\
\hline$v_{47}$ & $702(12,6) 0.05$ & 687 & $714,714^{\#}$ & $713,715^{\#}$ & $\begin{array}{c}\omega(\mathrm{COOH})(24)+\Phi_{1}(\mathrm{R})(16)-v\left(\mathrm{C}_{4}-\mathrm{C}_{11}\right)(10)-\delta(\mathrm{COOH}) \\
(8)-v\left(C_{11}-C_{12}\right)(7)-\mathrm{a}_{1}(R)(7)\end{array}$ & $v\left(C_{4}-C_{11}\right)$ \\
\hline $\mathrm{v}_{48}$ & $657(3,2) 0.66$ & 643 & - & $646^{\#}$ & $\begin{array}{c}\delta(\mathrm{COOH})(34)+\omega\left(\mathrm{NH}_{2}\right)(22)+\Phi_{1}(\mathrm{R})(10)+\omega(\mathrm{COOH}) \\
(10)\end{array}$ & $\delta(\mathrm{COOH})$ \\
\hline$v_{49}$ & $655(1,5) 0.75$ & 641 & $641,642^{\#}$ & 651 & $\alpha_{3}(R)(79)$ & $\alpha_{3}(R)$ \\
\hline$v_{50}$ & $565(11,2) 0.46$ & 553 & $574,574^{\#}$ & $576,575^{\#}$ & $\begin{array}{l}\rho(\mathrm{COOH})(16)+\gamma\left(\mathrm{C}_{1}-\mathrm{O}_{23}\right)(11)+\omega\left(\mathrm{NH}_{2}\right)(11)+\alpha\left(\mathrm{C}_{11^{-}}\right. \\
\left.\mathrm{C}_{12}-\mathrm{N}_{16}\right)(10)-\Phi_{2}(\mathrm{R})(8)-\alpha\left(\mathrm{C}_{4}-\mathrm{C}_{11}-\mathrm{C}_{12}\right)(6)-\mathrm{a}_{2}(\mathrm{R})(6)\end{array}$ & $\rho(\mathrm{COOH})$ \\
\hline$v_{51}$ & $526(14,1) 0.33$ & 515 & $528,534^{\#}$ & $530,535^{\#}$ & $\begin{array}{c}\mathrm{Y}\left(\mathrm{C}_{1}-\mathrm{O}_{23}\right)(27)+\mathrm{\gamma}\left(\mathrm{C}_{4}-\mathrm{C}_{11}\right)(13)-\Phi_{2}(\mathrm{R})(25)+\delta(\mathrm{COOH}) \\
(7)-\rho(\mathrm{COOH})(7)\end{array}$ & $\Phi_{2}(R)$ \\
\hline$v_{52}$ & $492(9,3) 0.40$ & 481 & $492,496^{\#}$ & $495,499^{\#}$ & $\alpha_{2}(R)(39)+y\left(C_{1}-O_{23}\right)(11)-\Phi_{2}(R)(10)$ & $\alpha_{2}(R)$ \\
\hline$v_{53}$ & $428(11,0) 0.59$ & 419 & - & $434,435^{\#}$ & $\beta\left(C_{1}-O_{23}\right)(46)-\alpha_{3}(R)(16)+\beta\left(C_{4}-C_{11}\right)(13)+\Phi_{3}(R)(11)$ & $\beta\left(\mathrm{C}_{1}-\mathrm{O}_{23}\right)$ \\
\hline$v_{54}$ & $423(3,0) 0.35$ & 414 & - & $408^{\#}$ & $\Phi_{3}(R)(76)$ & $\Phi_{3}(\mathrm{R})$ \\
\hline
\end{tabular}


Citation: Yadav RA, Dixit V, Yogesh M, Santhosh C (2015) Raman and IR Spectral and DFT Based Vibrational and Electronic Characterization of Isolated and Zwitterionic Forms of L-Tyrosine. Pharm Anal Acta 6: 439. doi:10.4172/21532435.1000439

Page 9 of 18

\begin{tabular}{|c|c|c|c|c|c|c|}
\hline$v_{55}$ & $409(4,3) 0.32$ & 400 & $380,386^{\#}$ & $388^{\#}$ & $\alpha\left(\mathrm{C}_{11}-\mathrm{C}_{12}-\mathrm{N}_{16}\right)(37)+\omega(\mathrm{COOH})(7)-\Phi_{1}(\mathrm{R})(6)$ & $\mathrm{a}\left(\mathrm{N}_{16}-\mathrm{C}_{12}-\mathrm{C}_{11}\right)$ \\
\hline$v_{56}$ & $347(7,3) 0.58$ & 340 & $338^{\#}$ & $340^{\#}$ & $\begin{array}{c}\alpha\left(C_{11}-C_{12}-N_{16}\right)(22)+\Phi_{1}(R)(16)-y\left(C_{4}-C_{11}\right)(10)+ \\
y\left(C_{1}-O_{23}\right)(10)\end{array}$ & $\mathrm{V}\left(\mathrm{C}_{1}-\mathrm{O}_{23}\right)$ \\
\hline$v_{57}$ & $340(8,2) 0.28$ & 333 & $336,331^{\#}$ & $327^{\#}$ & $\alpha\left(\mathrm{N}_{16}-\mathrm{C}_{12}-\mathrm{C}_{19}\right)(40)-\rho(\mathrm{COOH})(29)$ & $\mathrm{a}\left(\mathrm{N}_{16}-\mathrm{C}_{12}-\mathrm{C}_{19}\right)$ \\
\hline$v_{58}$ & $310(96,1) 0.71$ & 303 & $311,313^{\#}$ & $313^{\#}$ & $\mathrm{~T}\left(\mathrm{C}_{1}-\mathrm{O}_{23}\right)(89)$ & $\mathrm{T}\left(\mathrm{C}_{1}-\mathrm{O}_{23}\right)$ \\
\hline $\mathrm{v}_{59}$ & $307(15,0) 0.74$ & 300 & -- & -- & $\mathrm{T}\left(\mathrm{C}_{12}-\mathrm{N}_{16}\right)(41)-\mathrm{T}\left(\mathrm{C}_{19}-\mathrm{O}_{21}\right)(20)-\beta\left(\mathrm{C}_{4}-\mathrm{C}_{11}\right)(13)$ & $\beta\left(C_{4}-C_{11}\right)$ \\
\hline $\mathrm{v}_{60}$ & $296(27,0) 0.73$ & 290 & - & $294^{\#}$ & $\begin{array}{c}\mathrm{T}\left(\mathrm{C}_{12}-\mathrm{N}_{16}\right)(41)+\beta\left(\mathrm{C}_{4}-\mathrm{C}_{11}\right)(16)-\mathrm{T}\left(\mathrm{C}_{19}-\mathrm{O}_{21}\right)(8)-\beta\left(\mathrm{C}_{12}-\mathrm{C}_{19}\right) \\
(7)\end{array}$ & $\mathrm{T}\left(\mathrm{C}_{12}-\mathrm{N}_{16}\right)$ \\
\hline$v_{61}$ & $186(0,1) 0.13$ & 182 & $196^{*}$ & - & $\begin{array}{c}\Phi_{2}(R)(24)-\alpha\left(C_{11}-C_{12}-C_{19}\right)(16)-\alpha\left(C_{4}-C_{11}-C_{12}\right)(14)- \\
v\left(C_{4}-C_{11}\right)(6)-\gamma\left(C_{4}-C_{11}\right)(6)-\gamma\left(C_{12}-C_{19}\right)(6)\end{array}$ & $\alpha\left(C_{4}-C_{11}-C_{12}\right)$ \\
\hline $\mathrm{v}_{62}$ & $169(9,1) 0.66$ & 165 & $167^{\#}$ & -- & $\begin{array}{c}\alpha\left(C_{11}-C_{12}-C_{19}\right)(34)+ \\
+\Phi_{2}(R)(32)+\gamma\left(C_{12}-C_{19}\right)(7)-\alpha\left(C_{4}-\right. \\
\left.C_{11}-C_{12}\right)(7)\end{array}$ & $\alpha\left(C_{11}-C_{12}-C_{19}\right)$ \\
\hline$v_{63}$ & $83(1,1) 0.75$ & 81 & -- & -- & $\mathrm{T}\left(\mathrm{C}_{12}-\mathrm{C}_{19}\right)(58)-\mathrm{T}\left(\mathrm{C}_{12}-\mathrm{N}_{16}\right)(14)-\mathrm{T}\left(\mathrm{C}_{19}-\mathrm{O}_{21}\right)(10)$ & $\mathrm{T}\left(\mathrm{C}_{12}-\mathrm{C}_{19}\right)$ \\
\hline $\mathrm{v}_{64}$ & $63(1,1) 0.71$ & 62 & -- & -- & $\begin{array}{c}\mathrm{T}\left(\mathrm{C}_{12}-\mathrm{C}_{19}\right)(31)-\mathrm{y}\left(\mathrm{C}_{4}-\mathrm{C}_{11}\right)(14)-\alpha\left(\mathrm{C}_{4}-\mathrm{C}_{11}-\mathrm{C}_{12}\right)(12)-\Phi_{2}(R) \\
(9)-\mathrm{T}\left(\mathrm{C}_{12}-\mathrm{N}_{16}\right)(9)+\alpha\left(\mathrm{C}_{11}-\mathrm{C}_{12}-\mathrm{C}_{19}\right)(7)-\mathrm{T}\left(\mathrm{C}_{19}-\mathrm{O}_{21}\right)(6)\end{array}$ & $\mathrm{Y}\left(\mathrm{C}_{4}-\mathrm{C}_{11}\right)$ \\
\hline$v_{65}$ & $46(3,1) 0.75$ & 45 & -- & -- & $\mathrm{T}\left(\mathrm{C}_{11}-\mathrm{C}_{12}\right)(46)+\mathrm{T}\left(\mathrm{C}_{12}-\mathrm{C}_{19}\right)(20)-\mathrm{T}\left(\mathrm{C}_{12}-\mathrm{N}_{16}\right)(7)$ & $T\left(R^{\prime \prime}\right)$ \\
\hline$v_{66}$ & $40(1,4) 0.75$ & 39 & -- & -- & $T\left(C_{4}-C_{11}\right)(57)+T\left(C_{12}-C_{19}\right)(18)-T\left(C_{12}-N_{16}\right)(9)$ & $T\left(R^{\prime}\right)$ \\
\hline
\end{tabular}

The number before the modes are the $\%$ potential energy calculated using normal coordinate analysis. The modes contributing with $\leq 5 \%$ have been omitted. The abbreviations $v, \delta, \alpha(R), \Phi_{1}(R), v(R), \beta, \gamma, \alpha(A-B-C), \omega, \rho, \tau$ asym and sym have been used for stretching, scissoring, ring planar deformation, ring non-planar deformation, ring stretching, in plane bending, out of plane bending, angle bending, wagging, rocking, torsion, anti-symmetric and symmetric respectively.

Calculated wave numbers up to $1000 \mathrm{~cm}^{-1}$ were scaled by the scale factor 0.9786 and those above $1000 \mathrm{~cm}^{-1}$ by the scale factor 0.9550 for larger wave numbers.

Number outside bracket is frequency in $\mathrm{cm}^{-1}$ unit, numbers within the bracket are IR intensity (approximate) and Raman activity (approximate) and number outside bracket is depolarization ratio. The values superscripted with \# are taken from literature Ref.-[6]

Table 6: Observed and computed IR and Raman frequencies of the normal modes of vibration, PEDs and assignments for the most stable conformer of L-TYR in isolated molecule.

\begin{tabular}{|c|c|c|c|c|c|c|}
\hline \multirow[t]{2}{*}{ Mode } & \multicolumn{2}{|c|}{ Computed Frequencies } & \multicolumn{2}{|c|}{ Observed Freq. } & \multirow[t]{2}{*}{ PEDs } & \multirow[t]{2}{*}{ Mode Assignment } \\
\hline & Unscaled & Scaled & Raman & IR & & \\
\hline$v_{1}$ & $3811(144,239) 0.34$ & 3640 & - & $3430^{\#}$ & $\mathrm{v}\left(\mathrm{O}_{22}-\mathrm{H}_{23}\right)(100)$ & $\mathrm{v}\left(\mathrm{O}_{22}-\mathrm{H}_{23}\right)$ \\
\hline$v_{2}$ & $3526(134,72) 0.67$ & 3367 & - & - & $\mathrm{v}_{\text {asym }}\left(\mathrm{H}_{17}-\mathrm{N}_{16}-\mathrm{H}_{18}\right)(100)$ & $\mathrm{V}_{\text {asym }}\left(\mathrm{H}_{17}-\mathrm{N}_{16}-\mathrm{H}_{18}\right)$ \\
\hline$v_{3}$ & $3456(136,226) 0.06$ & 3300 & 3207 & 3207 & $\mathrm{v}_{\text {sym }}\left(\mathrm{H}_{17}-\mathrm{N}_{16}-\mathrm{H}_{18}\right)(99)$ & $\mathrm{v}_{\text {sym }}\left(\mathrm{H}_{17}-\mathrm{N}_{16}-\mathrm{H}_{18}\right)$ \\
\hline$v_{4}$ & $3190(10,360) 0.21$ & 3047 & - & - & $v\left(C_{6}-H_{10}\right)(92)+v\left(C_{5}-H_{9}\right)(6)$ & $\mathrm{v}(\mathrm{CH})$ \\
\hline$v_{5}$ & $3174(14,330) 0.15$ & 3031 & $3061^{\#}$ & $3041,3068^{\#}$ & $\mathrm{v}\left(\mathrm{C}_{3}-\mathrm{H}_{8}\right)(53)+\mathrm{v}\left(\mathrm{C}_{2}-\mathrm{H}_{7}\right)(45)$ & $\mathrm{v}(\mathrm{CH})$ \\
\hline$v_{6}$ & $3161(11,200) 0.52$ & 3019 & $3042^{\#}$ & $3025,3042^{\#}$ & $\mathrm{v}\left(\mathrm{C}_{5}-\mathrm{H}_{9}\right)(89)-\mathrm{v}\left(\mathrm{C}_{6}-\mathrm{H}_{10}\right)(6)$ & $\mathrm{v}(\mathrm{CH})$ \\
\hline$v_{7}$ & $3159(21,89) 0.75$ & 3017 & $3027^{\#}$ & $3024^{\#}$ & $\mathrm{v}\left(\mathrm{C}_{2}-\mathrm{H}_{7}\right)(53)-\mathrm{v}\left(\mathrm{C}_{3}-\mathrm{H}_{8}\right)(42)$ & $\mathrm{v}(\mathrm{CH})$ \\
\hline$v_{8}$ & $3127(44,66) 0.30$ & 2986 & $3014^{\#}$ & $3014^{\#}$ & $\mathrm{v}\left(\mathrm{C}_{12}-\mathrm{H}_{15}\right)(86)-\mathrm{v}\left(\mathrm{N}_{16}-\mathrm{H}_{24}\right)(10)$ & $\mathrm{v}\left(\mathrm{C}_{12}-\mathrm{H}_{15}\right)$ \\
\hline$v_{9}$ & $3106(414,146) 0.06$ & 2966 & $2950^{\#}$ & $2960,2962^{\#}$ & $\mathrm{v}\left(\mathrm{N}_{16}-\mathrm{H}_{24}\right)(88)+\mathrm{v}\left(\mathrm{C}_{12}-\mathrm{H}_{15}\right)(10)$ & $\mathrm{v}\left(\mathrm{N}_{16}-\mathrm{H}_{24}\right)$ \\
\hline $\mathrm{v}_{10}$ & $3093(6,162) 0.64$ & 2954 & $2923^{\#}$ & $2931,2928^{\#}$ & $\mathrm{v}_{\text {asym }}\left(\mathrm{CH}_{2}\right)(95)$ & $\mathrm{v}_{\text {asym }}\left(\mathrm{CH}_{2}\right)$ \\
\hline$v_{11}$ & $3047(23,299) 0.04$ & 2910 & $2863^{\#}$ & $2894,2864^{\#}$ & $\mathrm{v}_{\text {sym }}\left(\mathrm{CH}_{2}\right)(99)$ & $\mathrm{v}_{\text {sym }}\left(\mathrm{CH}_{2}\right)$ \\
\hline$v_{12}$ & $1669(317,21) 0.75$ & 1594 & - & 1630\# & $\begin{array}{c}\delta_{\mathrm{as}}\left(\mathrm{NH}_{3}\right)(40)+\mathrm{vas}_{\mathrm{as}}\left(\mathrm{CO}_{2}\right)(33)-\delta_{\mathrm{as}}^{\prime}\left(\mathrm{NH}_{3}\right)(16)- \\
\left.\mathrm{NH}_{3}\right)(5)\end{array}$ & $\delta_{a s}\left(\mathrm{NH}_{3}\right)$ \\
\hline$v_{13}$ & $1659(433,7) 0.75$ & 1584 & $1587,1590^{\#}$ & $1582,1589^{\#}$ & $\mathrm{v}_{\mathrm{as}}\left(\mathrm{CO}_{2}\right)(53)+\delta_{\mathrm{as}}^{\prime}\left(\mathrm{NH}_{3}\right)(34)+\rho\left(\mathrm{CO}_{2}\right)(5)$ & $\mathrm{v}_{\mathrm{as}}\left(\mathrm{CO}_{2}\right)$ \\
\hline $\mathrm{v}_{14}$ & $1647(78,133) 0.56$ & 1573 & 1567 & 1579 & $\begin{array}{c}v\left(C_{2}-C_{3}\right)(20)+v\left(C_{5}-C_{6}\right)(17)-v\left(C_{1}-C_{6}\right) \\
(13)-v\left(C_{3}-C_{4}\right)(11)+\alpha_{2}(R)(10)-\beta\left(C_{3}-H_{8}\right)(7)- \\
\beta\left(C_{6}-H_{10}\right)(5)\end{array}$ & $v(R)$ \\
\hline $\mathrm{v}_{15}$ & $1627(38,29) 0.63$ & 1554 & $1551,1531^{\#}$ & $1572,1532^{\#}$ & $\begin{array}{c}v\left(C_{1}-C_{2}\right)(20)+v\left(C_{4}-C_{5}\right)(20)-v\left(C_{1}-C_{6}\right)(15)- \\
\alpha_{3}(R)(8)-v\left(C_{3}-C_{4}\right)(7)-\beta\left(C_{5}-H_{9}\right)(6)\end{array}$ & $v(R)$ \\
\hline $\mathrm{v}_{16}$ & $1622(84,16) 0.65$ & 1549 & $1481,1463^{\#}$ & $1453,1454^{\#}$ & $\begin{array}{c}\delta_{a s}\left(\mathrm{NH}_{3}\right)(34)+\delta_{\mathrm{as}}^{\prime}\left(\mathrm{NH}_{3}\right)(32)+\delta_{\mathrm{s}}\left(\mathrm{NH}_{3}\right)(11)+ \\
\mathrm{v}\left(\mathrm{C}_{19}-\mathrm{O}_{20}\right)(8)\end{array}$ & $\delta^{\prime}{ }_{\text {as }}\left(\mathrm{NH}_{3}\right)$ \\
\hline $\mathrm{v}_{17}$ & $1538(164,4) 0.74$ & 1469 & -- & $1435,1438^{\#}$ & $\begin{array}{c}\beta\left(C_{2}-H_{7}\right)(17)+\beta\left(C_{3}-H_{8}\right)(14)-\beta\left(C_{5}-H_{9}\right)(12)+ \\
v\left(C_{3}-C_{4}\right)(11)-v\left(C_{1}-C_{2}\right)(11)-\beta\left(C_{6}-H_{10}\right)(9)- \\
v\left(C_{1}-C_{6}\right)(8)+v\left(C_{1}-O_{22}\right)(7)+v\left(C_{4}-C_{5}\right)(5)\end{array}$ & $\beta(\mathrm{CH})$ \\
\hline $\mathrm{v}_{18}$ & $1486(13,11) 0.75$ & 1419 & $1419,1414^{\#}$ & $1417,1416^{\#}$ & $\delta\left(\mathrm{CH}_{2}\right)(89)$ & $\delta\left(\mathrm{CH}_{2}\right)$ \\
\hline $\mathrm{v}_{19}$ & $1464(28,2) 0.75$ & 1398 & -- & $1363,1367^{\#}$ & $\begin{array}{c}v\left(C_{5}-C_{6}\right)(19)-\beta\left(C_{6}-H_{10}\right)(13)-v\left(C_{2}-C_{3}\right) \\
(13)-\alpha(C-O H)(8)-\beta\left(C_{2}-H_{7}\right)(7)+\beta\left(C_{3}-H_{8}\right) \\
(6)+\beta\left(C_{4}-C_{11}\right)(5)+\beta\left(C_{1}-O_{22}\right)(5)+v\left(C_{4}-C_{5}\right)(5)\end{array}$ & $v(R)$ \\
\hline $\mathrm{v}_{20}$ & $1423(566,20) 0.74$ & 1359 & $1365,1368^{\#}$ & $1332,1328^{\#}$ & $\delta_{s}\left(N_{3}\right)(71)-v\left(C_{19}-\mathrm{O}_{20}\right)(9)-\delta_{a s}\left(N_{3}\right)(6)$ & $\delta_{s}\left(\mathrm{NH}_{3}\right)$ \\
\hline $\mathrm{v}_{21}$ & $1393(35,26) 0.62$ & 1330 & $1327,1328^{\#}$ & - & $\begin{array}{l}\alpha\left(\mathrm{C}_{11}-\mathrm{C}_{12}-\mathrm{H}_{15}\right)(30)-\alpha\left(\mathrm{H}_{15}-\mathrm{C}_{12}-\mathrm{N}_{16}\right)(17)- \\
\omega\left(\mathrm{CH}_{2}\right)(12)+\rho\left(\mathrm{CH}_{2}\right)(9)+v\left(\mathrm{C}_{19}-\mathrm{O}_{21}\right)(7)\end{array}$ & $\alpha\left(C_{11}-C_{12}-H_{15}\right)$ \\
\hline $\mathrm{v}_{22}$ & $1364(103,4) 0.37$ & 1303 & - & $1286^{\#}$ & $\begin{array}{c}\beta\left(\mathrm{C}_{5}-\mathrm{H}_{9}\right)(19)+\beta\left(\mathrm{C}_{3}-\mathrm{H}_{8}\right)(12)+\omega\left(\mathrm{CH}_{2}\right)(11)+ \\
\alpha(C-O H)(10)-\alpha\left(\mathrm{H}_{15}-\mathrm{C}_{12}-\mathrm{C}_{19}\right)(8)+\beta\left(\mathrm{C}_{6}-\mathrm{H}_{10}\right) \\
(7)\end{array}$ & $\beta(\mathrm{CH})$ \\
\hline $\mathrm{v}_{23}$ & $1360(20,7) 0.20$ & 1298 & -- & 1267 & $\begin{array}{c}\omega\left(C_{2}\right)(16)+v\left(C_{2}-C_{3}\right)(11)+v\left(C_{4}-C_{5}\right)(7)+ \\
v\left(C_{1}-C_{6}\right)(6)-v\left(C_{5}-C_{6}\right)(6)-\alpha(C-O H)(6)-\alpha\left(H_{15}-\right. \\
\left.C_{12}-C_{19}\right)(6)-v\left(C_{1}-C_{2}\right)(6)-\beta\left(C_{3}-H_{8}\right)(5)- \\
v\left(C_{3}-C_{4}\right)(5)-\beta\left(C_{5}-H_{9}\right)(5)\end{array}$ & $v(R)$ \\
\hline
\end{tabular}


Citation: Yadav RA, Dixit V, Yogesh M, Santhosh C (2015) Raman and IR Spectral and DFT Based Vibrational and Electronic Characterization of Isolated and Zwitterionic Forms of L-Tyrosine. Pharm Anal Acta 6: 439. doi:10.4172/21532435.1000439

Page 10 of 18

\begin{tabular}{|c|c|c|c|c|c|c|}
\hline $\mathrm{v}_{24}$ & $1344(289,18) 0.19$ & 1284 & $1284,1286^{\#}$ & $1247,1246^{\#}$ & $\begin{array}{l}v_{s}\left(C O_{2}\right)(41)-\delta\left(C O_{2}\right)(10)-\alpha\left(C_{11}-C_{12}-H_{15}\right)(9)+ \\
\delta_{s}\left(N_{3}\right)(8)-v\left(C_{12}-C_{19}\right)(8)+\alpha\left(H_{15}-C_{12}-N_{16}\right)(7)\end{array}$ & $\mathrm{v}_{\mathrm{s}}\left(\mathrm{CO}_{2}\right)$ \\
\hline $\mathrm{v}_{25}$ & $1332(6,13) 0.61$ & 1272 & $1249,1247^{\#}$ & 1243 & $\begin{array}{c}\omega\left(\mathrm{CH}_{2}\right)(19)+v\left(\mathrm{C}_{3}-\mathrm{C}_{4}\right)(11)-v\left(\mathrm{C}_{4}-\mathrm{C}_{5}\right) \\
(10)-\beta\left(\mathrm{C}_{2}-\mathrm{H}_{7}\right)(8)+\rho\left(\mathrm{CH}_{2}\right)(5)+v\left(\mathrm{C}_{5}-\mathrm{C}_{6}\right) \\
(5)-v\left(\mathrm{C}_{1}-\mathrm{C}_{6}\right)(5)\end{array}$ & $\omega\left(\mathrm{CH}_{2}\right)$ \\
\hline $\mathrm{v}_{26}$ & $1275(5,33) 0.34$ & 1218 & $1214,1212^{\#}$ & $1214,1213^{\#}$ & $\begin{array}{c}\alpha\left(\mathrm{H}_{15}-\mathrm{C}_{12}-\mathrm{N}_{16}\right)(21)+\rho\left(\mathrm{CH}_{2}\right)(17)+\alpha\left(\mathrm{C}_{11}-\right. \\
\left.\mathrm{C}_{12}-\mathrm{H}_{15}\right)\left(17+\omega\left(\mathrm{CH}_{2}\right)(14)\right.\end{array}$ & $\alpha\left(H_{15}-C_{12}-N_{16}\right)$ \\
\hline $\mathrm{v}_{27}$ & $1268(173,65) 0.03$ & 1211 & $1164,1178^{\#}$ & $1176,1177^{\#}$ & $\begin{array}{l}v(\mathrm{C}-\mathrm{OH})(46)+\alpha_{1}(\mathrm{R})(10)-\mathrm{v}\left(\mathrm{C}_{1}-\mathrm{C}_{2}\right)(9)- \\
\mathrm{v}\left(\mathrm{C}_{5}-\mathrm{C}_{6}\right)(7)-\beta\left(\mathrm{C}_{3}-\mathrm{H}_{8}\right)(7)-\beta\left(\mathrm{C}_{2}-\mathrm{H}_{7}\right)(5)\end{array}$ & $\mathrm{v}(\mathrm{C}-\mathrm{OH})$ \\
\hline $\mathrm{v}_{28}$ & $1237(43,3) 0.72$ & 1181 & -- & $1155,1153^{\#}$ & $\begin{array}{c}\rho\left(\mathrm{CH}_{2}\right)(18)-\alpha\left(\mathrm{C}_{11}-\mathrm{C}_{12}-\mathrm{H}_{15}\right)(16)-\alpha\left(\mathrm{H}_{15}-\right. \\
\left.\mathrm{C}_{12}-\mathrm{N}_{16}\right)(8)-\rho_{1}\left(\mathrm{NH}_{3}\right)(8)+\rho_{2}\left(\mathrm{NH}_{3}\right)(7)+ \\
v\left(\mathrm{C}_{11}-\mathrm{C}_{12}\right)(7)-\alpha\left(\mathrm{C}_{11}-\mathrm{C}_{12}-\mathrm{N}_{16}\right)(5)+v\left(\mathrm{C}_{4}-\mathrm{C}_{5}\right)(5)\end{array}$ & $\rho(\mathrm{CH} 2)$ \\
\hline $\mathrm{v}_{29}$ & $1220(3,82) 0.05$ & 1165 & $1156,1150^{\#}$ & - & $\begin{array}{c}v\left(C_{4}-C_{11}\right)(38)-\alpha_{1}(R)(11)-v\left(C_{3}-C_{4}\right) \\
(9)-v\left(C_{5}-C_{6}\right)(7)-v\left(C_{2}-C_{3}\right)(5)+\omega\left(C_{2}\right)(5)- \\
\beta\left(C_{5}-H_{9}\right)(5)\end{array}$ & $v(R)$ \\
\hline $\mathrm{v}_{30}$ & $1194(2,7) 0.28$ & 1140 & $1114,1113^{\#}$ & - & $\begin{array}{c}\beta\left(\mathrm{C}_{2}-\mathrm{H}_{7}\right)(29)-\beta\left(\mathrm{C}_{3}-\mathrm{H}_{8}\right)(20)+\beta\left(\mathrm{C}_{5}-\mathrm{H}_{9}\right)(13)- \\
\beta\left(\mathrm{C}_{6}-\mathrm{H}_{10}\right)(11)-\mathrm{v}\left(\mathrm{C}_{2}-\mathrm{C}_{3}\right)(8)-\alpha(\mathrm{C}-\mathrm{OH})(6)+ \\
v\left(\mathrm{C}_{1}-\mathrm{C}_{2}\right)(5)\end{array}$ & $\beta(\mathrm{CH})$ \\
\hline $\mathrm{v}_{31}$ & $1187(275,30) 0.07$ & 1134 & $1100,1098^{\#}$ & $1113,1112^{\#}$ & $\begin{array}{c}\alpha(C-O H)(46)+v\left(C_{1}-C_{6}\right)(16)-\beta\left(C_{6}-H_{10}\right)(13)- \\
v\left(C_{1}-O_{22}\right)(10)\end{array}$ & $\alpha(\mathrm{C}-\mathrm{OH})$ \\
\hline $\mathrm{v}_{32}$ & $1141(34,3) 0.66$ & 1090 & - & 1099, 1099\# & $\begin{array}{c}\beta\left(\mathrm{C}_{5}-\mathrm{H}_{9}\right)(12)-\beta\left(\mathrm{C}_{6}-\mathrm{H}_{10}\right)(11)-\rho_{2}\left(\mathrm{NH}_{3}\right)(10) \\
-\mathrm{v}\left(\mathrm{C}_{5}-\mathrm{C}_{6}\right)(8)+\rho\left(\mathrm{CH}_{2}\right)(8)-\mathrm{v}\left(\mathrm{C}_{11}-\mathrm{C}_{12}\right)(7)+ \\
\rho_{1}\left(\mathrm{NH}_{3}\right)(7)+\beta\left(\mathrm{C}_{3}-\mathrm{H}_{8}\right)(7)+v\left(\mathrm{C}_{2}-\mathrm{C}_{3}\right)(6)- \\
\beta\left(\mathrm{C}_{2}-\mathrm{H}_{7}\right)(5)\end{array}$ & $\beta(\mathrm{CH})$ \\
\hline $\mathrm{v}_{33}$ & $1112(130,8) 0.33$ & 1062 & $1045,1046^{\#}$ & $1042,1046^{\#}$ & $\begin{array}{c}\rho_{2}\left(\mathrm{NH}_{3}\right)(24)+\alpha\left(\mathrm{C}_{11}-\mathrm{C}_{12}-\mathrm{H}_{15}\right)(8)+\alpha\left(\mathrm{H}_{15}-\right. \\
\left.\mathrm{C}_{12}-\mathrm{C}_{19}\right)(7)+v\left(\mathrm{C}_{11}-\mathrm{C}_{12}\right)(6)-\mathrm{v}\left(\mathrm{C}_{5}-\mathrm{C}_{6}\right)(6)- \\
\beta\left(\mathrm{C}_{6}-\mathrm{H}_{10}\right)(6)+\alpha\left(\mathrm{N}_{16}-\mathrm{C}_{12}-\mathrm{C}_{19}\right)(5)-\mathrm{v}\left(\mathrm{C}_{12}-\mathrm{N}_{16}\right) \\
(5)\end{array}$ & $\rho_{2}\left(\mathrm{NH}_{3}\right)$ \\
\hline $\mathrm{v}_{34}$ & $1084(17,6) 0.74$ & 1035 & $1017,1017^{\#}$ & $1016,1017^{\#}$ & $\begin{array}{c}\rho_{1}\left(\mathrm{NH}_{3}\right)(30)+\rho\left(\mathrm{CH}_{2}\right)(12)+\rho_{2}\left(\mathrm{NH}_{3}\right)(11)+ \\
\alpha\left(\mathrm{H}_{15}-\mathrm{C}_{12}-\mathrm{C}_{19}\right)(9)+\alpha\left(\mathrm{N}_{16}-\mathrm{C}_{12}-\mathrm{C}_{19}\right)(7)\end{array}$ & $\rho_{1}\left(\mathrm{NH}_{3}\right)$ \\
\hline $\mathrm{v}_{35}$ & $1038(38,30) 0.50$ & 991 & $986,985^{\#}$ & $986,988^{\#}$ & $\begin{array}{c}v\left(\mathrm{C}_{12}-\mathrm{N}_{16}\right)(30)-\mathrm{v}\left(\mathrm{C}_{11}-\mathrm{C}_{12}\right)(21)-\mathrm{T}(\mathrm{CH} 2)(7)+ \\
\rho_{2}\left(\mathrm{NH}_{3}\right)(7)+\mathrm{a}\left(\mathrm{C}_{4}-\mathrm{C}_{11}-\mathrm{C}_{12}\right)(6)\end{array}$ & $v\left(C_{11}-C_{12}\right)$ \\
\hline $\mathrm{v}_{36}$ & $1027(2,0) 0.12$ & 981 & - & 939, 939\# & $\begin{array}{c}\alpha_{1}(R)(50)+v\left(C_{1}-C_{2}\right)(11)+v\left(C_{1}-C_{6}\right)(10)- \\
v\left(C_{4}-C_{5}\right)(7)-v\left(C_{3}-C_{4}\right)(7)-\beta\left(C_{6}-H_{10}\right)(5)\end{array}$ & $\alpha_{1}(R)$ \\
\hline$v_{37}$ & $979(0,1) 0.34$ & 958 & $935,938^{\#}$ & - & $\begin{array}{c}\mathrm{Y}\left(\mathrm{C}_{5}-\mathrm{H}_{9}\right)(37)-\mathrm{Y}\left(\mathrm{C}_{3}-\mathrm{H}_{8}\right)(22)-\mathrm{\gamma}\left(\mathrm{C}_{6}-\mathrm{H}_{10}\right)(20)+ \\
\mathrm{Y}\left(\mathrm{C}_{2}-\mathrm{H}_{7}\right)(11)+\Phi_{3}(\mathrm{R})(8)\end{array}$ & $\mathrm{Y}(\mathrm{CH})$ \\
\hline $\mathrm{v}_{38}$ & $956(2,2) 0.72$ & 936 & $894,897^{\#}$ & - & $\begin{array}{c}Y\left(C_{3}-H_{8}\right)(31)+Y\left(C_{5}-H_{9}\right)(19)+\Phi_{1}(R)(17)- \\
Y\left(C_{2}-H_{7}\right)(16)-Y\left(C_{6}-H_{10}\right)(10)\end{array}$ & $\mathrm{Y}(\mathrm{CH})$ \\
\hline $\mathrm{v}_{39}$ & $943(20,8) 0.49$ & 923 & 878 & $896,899^{\#}$ & $\begin{array}{c}\mathrm{T}\left(\mathrm{CH}_{2}\right)(29)-\rho_{1}\left(\mathrm{NH}_{3}\right)(28)-v\left(\mathrm{C}_{11}-\mathrm{C}_{12}\right)(12)+ \\
\alpha\left(\mathrm{C}_{11}-\mathrm{C}_{12}-\mathrm{N}_{16}\right)(5)\end{array}$ & $\mathrm{T}\left(\mathrm{CH}_{2}\right)$ \\
\hline $\mathrm{v}_{40}$ & $896(146,25) 0.10$ & 877 & $855^{\#}$ & $878,878^{\#}$ & $\begin{array}{c}v\left(C_{12}-C_{19}\right)(32)+\delta\left(C O_{2}\right)(10)+\rho_{2}\left(N_{3}\right)(10)- \\
v\left(C_{11}-C_{12}\right)(7)-\alpha\left(C_{11}-C_{12}-C_{19}\right)(6)\end{array}$ & $v\left(C_{12}-C_{19}\right)$ \\
\hline $\mathrm{v}_{41}$ & $860(24,20) 0.01$ & 842 & $845,846^{\#}$ & $842,842^{\#}$ & $\begin{array}{c}\mathrm{y}\left(\mathrm{C}_{6}-\mathrm{H}_{10}\right)(16)+\mathrm{v}\left(\mathrm{C}_{12}-\mathrm{N}_{16}\right)(12)+\mathrm{v}\left(\mathrm{C}_{2}-\mathrm{H}_{7}\right) \\
(8)-\Phi_{2}(\mathrm{R})(6)+\mathrm{v}\left(\mathrm{C}_{5}-\mathrm{C}_{6}\right)(6)-\mathrm{v}\left(\mathrm{C}_{12}-\mathrm{C}_{19}\right)(6)+ \\
\mathrm{\gamma}\left(\mathrm{C}_{1}-\mathrm{O}_{22}\right)(5)-\mathrm{V}\left(\mathrm{C}_{4}-\mathrm{C}_{11}\right)(5)\end{array}$ & $v\left(C_{12}-N_{16}\right)$ \\
\hline$v_{42}$ & $849(12,45) 0.09$ & 831 & $828,829^{\#}$ & $829,830^{\#}$ & $\begin{array}{c}\mathrm{v}\left(\mathrm{C}_{6}-\mathrm{H}_{10}\right)(12)-\mathrm{a}_{2}(\mathrm{R})(11)-\mathrm{v}\left(\mathrm{C}_{1}-\mathrm{O}_{22}\right)(8) \\
)-\mathrm{v}\left(\mathrm{C}_{1}-\mathrm{C}_{2}\right)(7)-\mathrm{v}\left(\mathrm{C}_{1}-\mathrm{C}_{6}\right)(6)-\mathrm{v}\left(\mathrm{C}_{5}-\mathrm{C}_{6}\right)(5) \\
+\mathrm{y}\left(\mathrm{C}_{5}-\mathrm{H}_{9}\right)(6)\end{array}$ & $v(R)$ \\
\hline $\mathrm{v}_{43}$ & $830(12,8) 0.54$ & 812 & 808 & $803,808^{\#}$ & $\begin{array}{c}\mathrm{Y}\left(\mathrm{C}_{6}-\mathrm{H}_{10}\right)(35)+\mathrm{Y}\left(\mathrm{C}_{5}-\mathrm{H}_{9}\right)(19)-\mathrm{Y}\left(\mathrm{C}_{2}-\mathrm{H}_{7}\right)(11)- \\
\mathrm{v}\left(\mathrm{C}_{12}-\mathrm{N}_{16}\right)(9)-\mathrm{V}\left(\mathrm{C}_{3}-\mathrm{H}_{8}\right)(6)-\mathrm{T}(\mathrm{CH} 2)(5)\end{array}$ & $\mathrm{\gamma}(\mathrm{CH})$ \\
\hline $\mathrm{v}_{44}$ & $822(57,6) 0.11$ & 804 & $796,797^{\#}$ & $793,799^{\#}$ & $\begin{array}{c}\mathrm{Y}\left(\mathrm{C}_{2}-\mathrm{H}_{7}\right)(36)+\mathrm{Y}\left(\mathrm{C}_{3}-\mathrm{H}_{8}\right)(19)-\mathrm{v}\left(\mathrm{C}_{12}-\mathrm{N}_{16}\right)(10)+ \\
\mathrm{Y}\left(\mathrm{C}_{1}-\mathrm{O}_{22}\right)(8)-\mathrm{T}(\mathrm{CH} 2)(7)\end{array}$ & $\mathrm{Y}(\mathrm{CH})$ \\
\hline $\mathrm{v}_{45}$ & $806(74,22) 0.02$ & 789 & $741,738^{\#}$ & $741,739^{\#}$ & $\begin{array}{c}\delta\left(\mathrm{CO}_{2}\right)(25)-\alpha_{1}(\mathrm{R})(12)+\omega\left(\mathrm{CO}_{2}\right)(10)+ \\
v\left(\mathrm{C}_{1}-\mathrm{O}_{22}\right)(10)+v\left(\mathrm{C}_{11}-\mathrm{C}_{12}\right)(6)+v\left(\mathrm{C}_{12}-\mathrm{C}_{19}\right)(5)\end{array}$ & $\delta\left(\mathrm{CO}_{2}\right)$ \\
\hline$v_{46}$ & $737(5,5) 0.64$ & 721 & $714,714^{\#}$ & $713,715^{\#}$ & $\begin{array}{c}\Phi_{1}(\mathrm{R})(53)+\omega\left(\mathrm{CO}_{2}\right)(11)-\mathrm{Y}\left(\mathrm{C}_{1}-\mathrm{O}_{22}\right)(11)- \\
\mathrm{Y}\left(\mathrm{C}_{4}-\mathrm{C}_{11}\right)(11)\end{array}$ & $\Phi_{1}(\mathrm{R})$ \\
\hline $\mathrm{v}_{47}$ & $721(2,6) 0.10$ & 706 & - & - & $\begin{array}{c}\Phi_{1}(R)(29)-v\left(C_{4}-C_{11}\right)(11)-\delta\left(C_{2}\right)(11) \\
-a_{1}(R)(9)-\omega\left(C_{2}\right)(6)-v\left(C_{1}-O_{22}\right)(5)+ \\
v\left(C_{1}-O_{22}\right)(5)\end{array}$ & $v\left(C_{4}-C_{11}\right)$ \\
\hline $\mathrm{v}_{48}$ & $676(13,7) 0.44$ & 662 & $641,642^{\#}$ & $646^{\#}$ & $\begin{array}{l}\Phi_{1}(\mathrm{R})(20)+\delta\left(\mathrm{CO}_{2}\right)(17)-\omega\left(\mathrm{CO}_{2}\right)(16)+\alpha\left(\mathrm{N}_{16}-\right. \\
\left.\mathrm{C}_{12}-\mathrm{C}_{19}\right)(11)-\mathrm{v}\left(\mathrm{C}_{12}-\mathrm{C}_{19}\right)(6)+\alpha\left(\mathrm{C}_{4}-\mathrm{C}_{11}-\mathrm{C}_{12}\right)(6)\end{array}$ & $\omega\left(\mathrm{CO}_{2}\right)$ \\
\hline $\mathrm{v}_{49}$ & $654(1,12) 0.71$ & 640 & $574,574^{\#}$ & 651 & $\alpha_{3}(R)(78)+\beta\left(C_{1}-O_{22}\right)(5)$ & $\alpha_{3}(R)$ \\
\hline $\mathrm{v}_{50}$ & $557(6,2) 0.48$ & 545 & -- & $576,575^{\#}$ & $\begin{array}{c}\left.\gamma\left(C_{1}-O_{22}\right)(16)\right)-\Phi_{2}(R)(13)+\rho\left(C_{2}\right)(12)- \\
\alpha_{2}(R)(10)-\gamma\left(C_{4}-C_{11}\right)(8)+\alpha\left(N_{16}-C_{12}-C_{19}\right)(8)+ \\
\alpha\left(C_{11}-C_{12}-N_{16}\right)(6)-\alpha\left(C_{4}-C_{11}-C_{12}\right)(6)\end{array}$ & $\mathrm{V}\left(\mathrm{C}_{4}-\mathrm{C}_{11}\right)$ \\
\hline $\mathrm{v}_{51}$ & $522(60,3) 0.16$ & 511 & $528,534^{\#}$ & $530,535^{\#}$ & $\begin{array}{c}\mathrm{v}\left(\mathrm{C}_{1}-\mathrm{O}_{22}\right)(25)-\Phi_{2}(\mathrm{R})(23)-\mathrm{\gamma}\left(\mathrm{C}_{4}-\mathrm{C}_{11}\right)(12)- \\
\rho\left(\mathrm{CO}_{2}\right)(11)\end{array}$ & $\mathrm{V}\left(\mathrm{C}_{1}-\mathrm{O}_{22}\right)$ \\
\hline $\mathrm{v}_{52}$ & $490(37,3) 0.57$ & 480 & $492,496^{\#}$ & $495,499^{\#}$ & $\begin{array}{c}\alpha_{2}(R)(37)+y\left(C_{1}-O_{22}\right)(7)+v\left(C_{12}-C_{19}\right)(7)+ \\
\rho\left(C_{2}\right)(7)-\Phi_{2}(R)(7)\end{array}$ & $a_{2}(R)$ \\
\hline
\end{tabular}


Citation: Yadav RA, Dixit V, Yogesh M, Santhosh C (2015) Raman and IR Spectral and DFT Based Vibrational and Electronic Characterization of Isolated and Zwitterionic Forms of L-Tyrosine. Pharm Anal Acta 6: 439. doi:10.4172/21532435.1000439

Page 11 of 18

\begin{tabular}{|c|c|c|c|c|c|c|}
\hline$v_{53}$ & $426(8,1) 0.20$ & 417 & $455,457^{\#}$ & $434,435^{\#}$ & $\begin{array}{c}\Phi_{3}(R)(38)+\beta\left(C_{1}-O_{22}\right)(29)-\alpha_{3}(R)(10)- \\
\beta\left(C_{4}-C_{11}\right)(8)\end{array}$ & $\beta\left(\mathrm{C}_{1}-\mathrm{O}_{22}\right)$ \\
\hline$v_{54}$ & $424(6,0) 0.61$ & 415 & -- & $408^{\#}$ & $\Phi_{3}(R)(63)-\beta\left(C_{1}-O_{22}\right)(12)$ & $\Phi_{3}(R)$ \\
\hline$v_{55}$ & $402(15,8) 0.37$ & 393 & $380,386^{\#}$ & $388^{\#}$ & $\begin{array}{c}\alpha\left(C_{11}-C_{12}-N_{16}\right)(33)+\alpha\left(C_{11}-C_{12}-C_{19}\right)(11)- \\
\Phi_{1}(R)(8)+\alpha_{2}(R)(5)\end{array}$ & $\alpha\left(C_{11}-C_{12}-N_{16}\right)$ \\
\hline$v_{56}$ & $341(22,4) 0.27$ & 334 & $338^{\#}$ & $340^{\#}$ & $\begin{array}{c}\alpha\left(C_{11}-C_{12}-N_{16}\right)(23)-\rho\left(C O_{2}\right)(10)+\Phi_{1}(R)(8)+ \\
\alpha\left(N_{16}-C_{12}-C_{19}\right)(7)-\beta\left(C_{4}-C_{11}\right)(6)-\beta\left(C_{1}-O_{22}\right) \\
(5)+\gamma\left(C_{1}-O_{22}\right)(5)\end{array}$ & $\rho\left(\mathrm{CO}_{2}\right)$ \\
\hline$v_{57}$ & $329(82,6) 0.58$ & 322 & $336,331^{\#}$ & $327^{*}$ & $\begin{array}{c}\alpha\left(\mathrm{N}_{16}-\mathrm{C}_{12}-\mathrm{C}_{19}\right)(32)-\rho\left(\mathrm{CO}_{2}\right)(16)+\mathrm{T}\left(\mathrm{NH}_{3}\right)(6)- \\
\rho_{2}\left(\mathrm{NH}_{3}\right)(6)+\mathrm{T}(\mathrm{OH})(6)-\mathrm{V}\left(\mathrm{C}_{4}-\mathrm{C}_{11}\right)(5)\end{array}$ & $\alpha\left(N_{16}-C_{12}-C_{19}\right)$ \\
\hline$v_{58}$ & $321(159,0) 0.74$ & 314 & $311,313^{\#}$ & $313^{\#}$ & $\mathrm{~T}(\mathrm{OH})(87)$ & $\mathrm{T}(\mathrm{OH})$ \\
\hline $\mathrm{v}_{59}$ & $302(4,1) 0.75$ & 296 & -- & -- & $\begin{array}{l}\beta\left(C_{4}-C_{11}\right)(38)+\beta\left(C_{1}-O_{22}\right)(12)+\alpha\left(C_{11}-\right. \\
\left.C_{12}-N_{16}\right)(7)+T\left(R^{\prime \prime}\right)(5)+Y\left(C_{4}-C_{11}\right)(5)\end{array}$ & $\beta\left(C_{4}-C_{11}\right)$ \\
\hline$v_{60}$ & $255(26,4) 0.41$ & 250 & $256,257^{\#}$ & $294^{\#}$ & $\mathrm{~T}\left(\mathrm{NH}_{3}\right)(62)+\delta_{\text {as }}^{\prime}\left(\mathrm{NH}_{3}\right)(12)-\alpha\left(\mathrm{N}_{16}-\mathrm{C}_{12}-\mathrm{C}_{19}\right)$ & $\mathrm{T}\left(\mathrm{NH}_{3}\right)$ \\
\hline$v_{61}$ & $183(4,2) 0.30$ & 179 & $196^{*}$ & -- & $\begin{array}{c}\alpha\left(C_{11}-C_{12}-C_{19}\right)(24)-\Phi_{2}(R)(12)+\alpha\left(C_{4}-C_{11}-C_{12}\right) \\
(9)+v\left(C_{12}-C_{19}\right)(8)+v\left(C_{4}-C_{11}\right)(7)-\omega\left(C_{2}\right) \\
(6)-\alpha, \alpha(R)(5)\end{array}$ & $\alpha\left(C_{11}-C_{12}-C_{19}\right)$ \\
\hline$v_{62}$ & $164(36,1) 0.74$ & 160 & $167^{\#}$ & -- & $\Phi_{2}(R)(41)+\alpha\left(C_{11}-C_{12}-C_{19}\right)(22)-\alpha\left(C_{4}-C_{11}-C_{12}\right)$ & $\Phi_{2}(R)$ \\
\hline$v_{63}$ & $77(1,3) 0.75$ & 75 & -- & -- & $\mathrm{T}\left(\mathrm{CO}_{2}\right)(40)-\mathrm{T}\left(\mathrm{NH}_{3}\right)(31)+\mathrm{T}\left(\mathrm{R}^{\prime}\right)(10)$ & $\mathrm{T}\left(\mathrm{CO}_{2}\right)$ \\
\hline $\mathrm{v}_{64}$ & $62(19,2) 0.71$ & 61 & -- & -- & $\begin{array}{c}\mathrm{T}\left(\mathrm{CO}_{2}\right)(31)-\mathrm{T}\left(\mathrm{NH}_{3}\right)(25)-\alpha\left(\mathrm{C}_{11}-\mathrm{C}_{12}-\mathrm{C}_{19}\right)(11)+ \\
\alpha\left(\mathrm{C}_{4}-\mathrm{C}_{11}-\mathrm{C}_{12}\right)(7)-\mathrm{Y}\left(\mathrm{C}_{4}-\mathrm{C}_{11}\right)(6)+\Phi_{2}(\mathrm{R})(5)\end{array}$ & $a\left(C_{4}-C_{11}-C_{12}\right)$ \\
\hline$v_{65}$ & $47(25,4) 0.75$ & 46 & -- & -- & $\begin{array}{c}T\left(R^{\prime \prime}\right)(58)+T\left(\mathrm{CO}_{2}\right)(13)-T\left(\mathrm{NH}_{3}\right)(10)+T\left(\mathrm{R}^{\prime}\right) \\
(6)\end{array}$ & $T\left(R^{\prime \prime}\right)$ \\
\hline$v_{66}$ & $40(8,7) 0.75$ & 39 & -- & -- & $\begin{array}{c}T\left(R^{\prime}\right)(38)+T\left(C_{2}\right)(15)-T\left(N_{3}\right)(14)+ \\
Y\left(C_{4}-C_{11}\right)(10)-T\left(R^{\prime \prime}\right)(8)\end{array}$ & $T\left(R^{\prime}\right)$ \\
\hline
\end{tabular}

The number before the modes are the $\%$ potential energy calculated using normal coordinate analysis. The modes contributing with $\leq 5 \%$ have been omitted. The abbreviations $v, \delta, \alpha(R), \Phi_{1}(R), v(R), \beta, \gamma, \alpha(A-B-C), \omega, \rho, \tau$ asym and sym have been used for stretching, scissoring, ring planar deformation, ring non-planar deformation, ring stretching, in plane bending, out of plane bending, angle bending, wagging, rocking, torsion, anti-symmetric and symmetric respectively.

Calculated wave numbers up to $1000 \mathrm{~cm}^{-1}$ were scaled by the scale factor 0.9786 and those above $1000 \mathrm{~cm}^{-1}$ by the scale factor 0.9550 for larger wave numbers.

Number outside bracket is frequency in $\mathrm{cm}^{-1}$ unit, numbers within the bracket are IR intensity (approximate) and Raman activity (approximate) and number outside bracket is depolarization ratio. The values superscripted with \# are taken from literature Ref.-[6]

Table 7: Observed and computed IR and Raman frequencies of the normal modes of vibration, PEDs and assignments for the most stable conformer of L-TYR in zwitterionic form.

\begin{tabular}{|c|c|c|c|c|}
\hline Donor NBOs (i) & Acceptor NBOs (j) & $\mathrm{E}(2) \mathrm{kcal} / \mathrm{mol}$ & $E(j)-E(i)$ (a.u.) & $F(i, j)($ a.u. $)$ \\
\hline$\sigma\left(C_{1}-C_{2}\right)$ & $\sigma^{*}\left(C_{1}-C_{6}\right)$ & 4.16 & 1.27 & 0.065 \\
\hline$\pi\left(C_{1}-C_{2}\right)$ & $\pi^{*}\left(C_{3}-C_{4}\right)$ & 21.63 & 0.30 & 0.072 \\
\hline$\pi\left(C_{1}-C_{2}\right)$ & $\pi^{*}\left(C_{5}-C_{6}\right)$ & 16.56 & 0.30 & 0.063 \\
\hline$\sigma\left(\mathrm{C}_{2}-\mathrm{C}_{3}\right)$ & $\sigma^{*}\left(\mathrm{C}_{1}-\mathrm{O}_{23}\right)$ & 4.43 & 1.05 & 0.061 \\
\hline$\pi\left(C_{3}-C_{4}\right)$ & $\pi^{*}\left(C_{1}-C_{2}\right)$ & 17.85 & 0.28 & 0.064 \\
\hline$\pi\left(C_{3}-C_{4}\right)$ & $\pi^{*}\left(C_{5}-C_{6}\right)$ & 20.93 & 0.29 & 0.069 \\
\hline$\sigma\left(\mathrm{C}_{3}-\mathrm{H}_{8}\right)$ & $\sigma^{*}\left(C_{4}-C_{5}\right)$ & 4.59 & 1.09 & 0.063 \\
\hline$\sigma\left(C_{5}-C_{6}\right)$ & $\pi^{*}\left(C_{1}-C_{2}\right)$ & 22.07 & 0.28 & 0.071 \\
\hline$\sigma\left(C_{5}-C_{6}\right)$ & $\pi^{*}\left(C_{3}-C_{4}\right)$ & 18.33 & 0.29 & 0.066 \\
\hline$\sigma\left(\mathrm{C}_{5}-\mathrm{H}_{9}\right)$ & $\sigma^{*}\left(C_{3}-C_{4}\right)$ & 4.57 & 1.10 & 0.063 \\
\hline$\sigma\left(\mathrm{C}_{6}-\mathrm{H}_{10}\right)$ & $\sigma^{*}\left(C_{1}-C_{2}\right)$ & 4.04 & 1.08 & 0.059 \\
\hline$\sigma\left(\mathrm{C}_{11}-\mathrm{H}_{13}\right)$ & $\sigma^{*}\left(C_{4}-C_{5}\right)$ & 4.42 & 1.07 & 0.061 \\
\hline$\sigma\left(\mathrm{C}_{11}-\mathrm{H}_{14}\right)$ & $\sigma^{*}\left(\mathrm{C}_{12}-\mathrm{N}_{16}\right)$ & 4.03 & 0.82 & 0.051 \\
\hline$\sigma\left(\mathrm{O}_{21}-\mathrm{H}_{22}\right)$ & $\sigma^{*}\left(\mathrm{C}_{19}-\mathrm{O}_{20}\right)$ & 6.45 & 1.38 & 0.084 \\
\hline$\sigma\left(\mathrm{O}_{23}-\mathrm{H}_{24}\right)$ & $\sigma^{*}\left(C_{1}-C_{6}\right)$ & 4.27 & 1.30 & 0.067 \\
\hline $\mathrm{CR}(1) \mathrm{O}_{20}$ & $R Y^{*}(1) C_{19}$ & 7.14 & 19.88 & 0.338 \\
\hline $\operatorname{LP}(1) \mathrm{N}_{16}$ & $\sigma^{*}\left(\mathrm{C}_{12}-\mathrm{H}_{15}\right)$ & 5.05 & 0.74 & 0.055 \\
\hline $\operatorname{LP}(1) \mathrm{N}_{16}$ & $\sigma^{*}\left(C_{12}-C_{19}\right)$ & 4.40 & 0.70 & 0.050 \\
\hline $\operatorname{LP}(1) \mathrm{N}_{16}$ & $\sigma^{*}\left(\mathrm{O}_{21}-\mathrm{O}_{22}\right)$ & 10.34 & 0.74 & 0.079 \\
\hline $\mathrm{LP}(1) \mathrm{O}_{20}$ & $R Y^{*}(1) C_{19}$ & 17.90 & 1.74 & 0.158 \\
\hline $\mathrm{LP}(2) \mathrm{O}_{20}$ & $\sigma^{*}\left(C_{12}-C_{19}\right)$ & 19.05 & 0.60 & 0.097 \\
\hline $\mathrm{LP}(2) \mathrm{O}_{20}$ & $\sigma^{*}\left(\mathrm{C}_{19}-\mathrm{O}_{21}\right)$ & 31.61 & 0.63 & 0.128 \\
\hline $\operatorname{LP}(1) \mathrm{O}_{21}$ & $\sigma^{*}\left(C_{12}-C_{19}\right)$ & 4.73 & 0.96 & 0.061 \\
\hline $\mathrm{LP}(2) \mathrm{O}_{21}$ & $\pi^{*}\left(\mathrm{C}_{19}-\mathrm{O}_{20}\right)$ & 48.56 & 0.34 & 0.116 \\
\hline $\mathrm{LP}(1) \mathrm{O}_{23}$ & $\sigma^{\star}\left(C_{1}-C_{2}\right)$ & 6.04 & 1.17 & 0.075 \\
\hline $\mathrm{LP}(2) \mathrm{O}_{23}$ & $\pi^{*}\left(C_{1}-C_{2}\right)$ & 27.68 & 0.35 & 0.094 \\
\hline$\pi^{*}\left(C_{1}-C_{2}\right)$ & $\pi^{*}\left(C_{3}-C_{4}\right)$ & 266.58 & 0.01 & 0.079 \\
\hline$\Pi^{*}\left(C_{1}-C_{2}\right)$ & $\pi^{*}\left(\mathrm{C}_{5}-\mathrm{C}_{6}\right)$ & 289.41 & 0.01 & 0.082 \\
\hline
\end{tabular}

Table 8: Second order perturbation theory analysis of Fock Matrix in the NBO basis of L-TYR (Isolated Molecule). 
Citation: Yadav RA, Dixit V, Yogesh M, Santhosh C (2015) Raman and IR Spectral and DFT Based Vibrational and Electronic Characterization of Isolated and Zwitterionic Forms of L-Tyrosine. Pharm Anal Acta 6: 439. doi:10.4172/21532435.1000439

Page 12 of 18

\begin{tabular}{|c|c|c|c|c|}
\hline Donor NBOs (i) & Acceptor NBOs (j) & $\mathrm{E}(2) \mathrm{kcal} / \mathrm{mol}$ & $E(j)-E(i)$ (a.u.) & $F(i, j)($ a.u. $)$ \\
\hline$\sigma\left(\mathrm{C}_{1}-\mathrm{C}_{2}\right)$ & $\sigma^{*}\left(C_{1}-C_{6}\right)$ & 4.13 & 1.27 & 0.065 \\
\hline$\sigma\left(\mathrm{C}_{2}-\mathrm{C}_{3}\right)$ & $\sigma^{*}\left(\mathrm{C}_{1}-\mathrm{O}_{22}\right)$ & 4.26 & 1.05 & 0.060 \\
\hline$\pi\left(\mathrm{C}_{2}-\mathrm{C}_{3}\right)$ & $\mathrm{LP}^{*}(1) \mathrm{C}_{1}$ & 53.69 & 0.14 & 0.094 \\
\hline$\pi\left(\mathrm{C}_{2}-\mathrm{C}_{3}\right)$ & $\mathrm{LP}(1) \mathrm{C}_{4}$ & 41.08 & 0.15 & 0.088 \\
\hline$\sigma\left(\mathrm{C}_{3}-\mathrm{H}_{8}\right)$ & $\sigma^{*}\left(C_{4}-C_{5}\right)$ & 4.60 & 1.09 & 0.063 \\
\hline$\pi\left(\mathrm{C}_{5}-\mathrm{C}_{6}\right)$ & $\mathrm{LP}^{*}(1) \mathrm{C}_{1}$ & 54.09 & 0.13 & 0.093 \\
\hline$\pi\left(\mathrm{C}_{5}-\mathrm{C}_{6}\right)$ & $\mathrm{LP}(1) \mathrm{C}_{4}$ & 44.40 & .15 & 0.090 \\
\hline$\sigma\left(\mathrm{C}_{5}-\mathrm{H}_{9}\right)$ & $\sigma^{*}\left(C_{3}-C_{4}\right)$ & 4.62 & 1.09 & 0.063 \\
\hline$\sigma\left(\mathrm{C}_{6}-\mathrm{H}_{10}\right)$ & $\sigma^{*}\left(C_{1}-C_{2}\right)$ & 4.08 & 1.07 & 0.059 \\
\hline$\sigma\left(\mathrm{C}_{11}-\mathrm{H}_{13}\right)$ & $\sigma^{*}\left(\mathrm{C}_{12}-\mathrm{N}_{16}\right)$ & 5.44 & 0.73 & 0.056 \\
\hline$\sigma\left(\mathrm{C}_{11}-\mathrm{H}_{14}\right)$ & $\sigma^{*}\left(\mathrm{C}_{3}-\mathrm{C}_{4}\right)$ & 4.57 & 1.07 & 0.062 \\
\hline$\sigma\left(\mathrm{O}_{22}-\mathrm{H}_{23}\right)$ & $\sigma^{*}\left(C_{1}-C_{6}\right)$ & 4.28 & 1.30 & 0.067 \\
\hline $\mathrm{CR}(1) \mathrm{O}_{21}$ & $R Y^{*}(1) C_{19}$ & 4.09 & 19.94 & 0.256 \\
\hline $\operatorname{LP}^{*}(1) \mathrm{C}_{1}$ & $\pi^{*}\left(C_{2}-C_{3}\right)$ & 61.01 & 0.15 & 0.104 \\
\hline $\mathrm{LP}^{*}(1) \mathrm{C}_{1}$ & $\pi^{*}\left(C_{5}-C_{6}\right)$ & 62.06 & 0.15 & 0.105 \\
\hline $\mathrm{LP}(1) \mathrm{C}_{4}$ & $\pi^{*}\left(C_{2}-C_{3}\right)$ & 77.03 & 0.13 & 0.109 \\
\hline $\mathrm{LP}(1) \mathrm{C}_{4}$ & $\pi^{*}\left(\mathrm{C}_{5}-\mathrm{C}_{6}\right)$ & 75.71 & .14 & 0.108 \\
\hline $\operatorname{LP}(1) C_{4}$ & $\sigma^{*}\left(C_{11}-C_{12}\right)$ & 5.74 & .48 & 0.064 \\
\hline $\operatorname{LP}(1) \mathrm{O}_{20}$ & $\mathrm{RY}^{*}(1) \mathrm{C}_{19}$ & 9.63 & 1.78 & 0.117 \\
\hline $\mathrm{LP}(2) \mathrm{O}_{20}$ & $\sigma^{*}\left(C_{12}-C_{19}\right)$ & 20.63 & 0.55 & 0.095 \\
\hline $\mathrm{LP}(2) \mathrm{O}_{20}$ & $\sigma^{*}\left(\mathrm{C}_{19}-\mathrm{O}_{21}\right)$ & 20.31 & 0.77 & 0.114 \\
\hline $\mathrm{LP}(1) \mathrm{O}_{21}$ & $R Y^{*}(1) C_{19}$ & 8.90 & 1.79 & 0.113 \\
\hline $\operatorname{LP}(2) \mathrm{O}_{21}$ & $\sigma^{*}\left(C_{12}-C_{19}\right)$ & 16.37 & 0.58 & 0.088 \\
\hline $\operatorname{LP}(2) \mathrm{O}_{21}$ & $\sigma^{*}\left(N_{16}-H_{24}\right)$ & 14.22 & 0.54 & 0.080 \\
\hline $\mathrm{LP}(2) \mathrm{O}_{21}$ & $\sigma^{*}\left(C_{19}-O_{20}\right)$ & 17.87 & 0.85 & 0.114 \\
\hline $\mathrm{LP}(3) \mathrm{O}_{21}$ & $\Pi^{*}\left(\mathrm{C}_{19}-\mathrm{O}_{20}\right)$ & 95.87 & 0.27 & 0.143 \\
\hline $\operatorname{LP}(1) \mathrm{O}_{22}$ & $\sigma^{*}\left(C_{1}-C_{2}\right)$ & 5.99 & 1.16 & 0.075 \\
\hline $\mathrm{LP}(2) \mathrm{O}_{22}$ & $\mathrm{LP}^{*}(1) \mathrm{C}_{1}$ & 49.41 & 0.20 & 0.118 \\
\hline
\end{tabular}

Table 9: Second order perturbation theory analysis of Fock Matrix in the NBO basis of L-TYR (Zwitterionic).

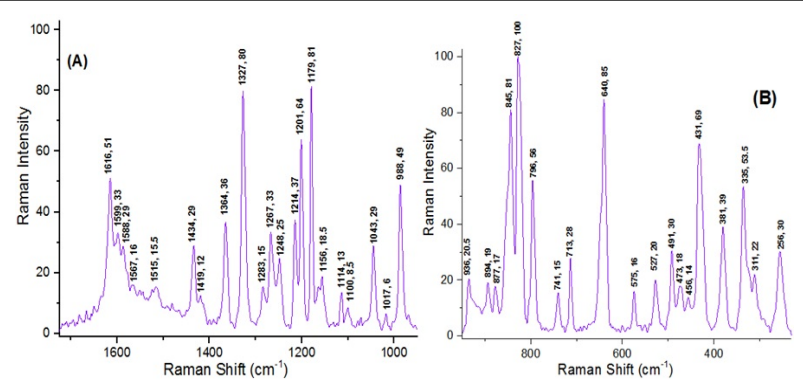

Figure 2: Observed Raman spectrum of the L-TYR.

$\mathrm{cm}^{-1}$ which appears to be arising due to some combination or overtone mode. The intense Raman peak observed at $1327 \mathrm{~cm}^{-1}$, assigned to the symmetric $\mathrm{CO}_{2}$ stretching mode, seems to give rise to the IR peak at $\sim 2075 \mathrm{~cm}^{-1}$ in combination with the $\mathrm{CO}_{2}$ scissoring mode. Thus the presence of $2075 \mathrm{~cm}^{-1}$ appears to be one of the signatures of the existence of the molecule in the zwitterionic form. These observations inspired us to investigate the vibrational spectra of isolated as well as zwitterionic form of L-TYR. The normal mode assignments could be discussed under the following three sections: (i) phenyl ring modes (30), (ii) $\mathrm{OH}$ group modes (3) and (iii) side carbon chain modes (33).

Phenyl Ring Modes [30]: The ring stretching modes $v(\mathrm{R})$ have been identified at the computed frequencies $1579\left(v_{14}\right), 1556\left(v_{15}\right)$, $1400\left(v_{18}\right), 1284\left(v_{23}\right), 1170\left(v_{27}\right)$ and $828\left(v_{42}\right) \mathrm{cm}^{-1}$ in isolated molecule while these have been observed in zwitterion at frequencies $1573\left(v_{14}\right)$, $1554\left(v_{15}\right), 1398\left(v_{19}\right), 1298\left(v_{23}\right), 1165\left(v_{29}\right)$ and $831\left(v_{42}\right) \mathrm{cm}^{-1}$. The ring stretching modes had been assigned at the frequencies 1618, 1572, $1296,1235,1126$ and $1003 \mathrm{~cm}^{-1}$ by Layton et al. [35] in zwitterionic
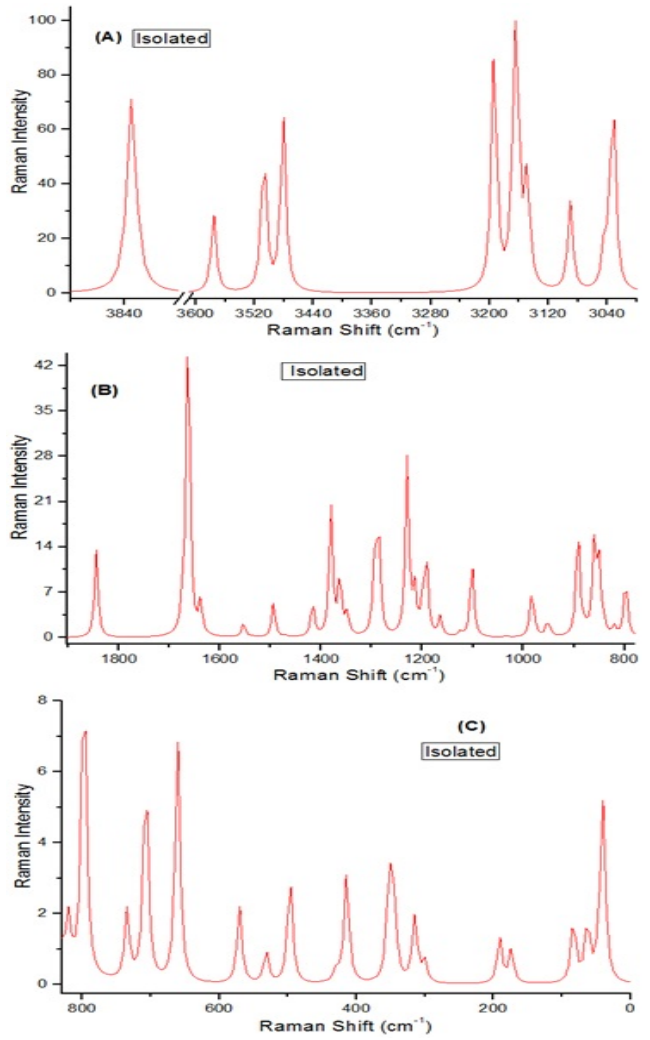

Figure 3(a): Computed Raman spectrum of the isolated molecule of L-TYR. 

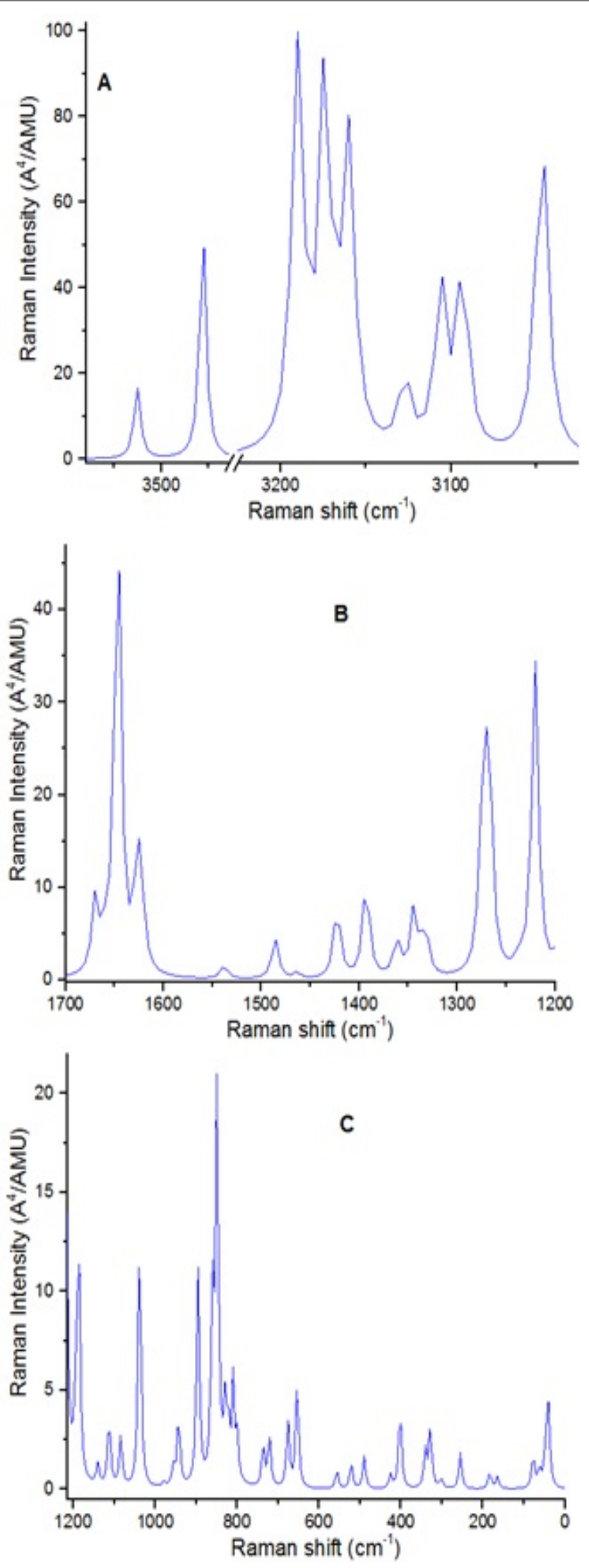

Figure 3(b): Computed Raman spectrum of the zwitterion of L-TYR.
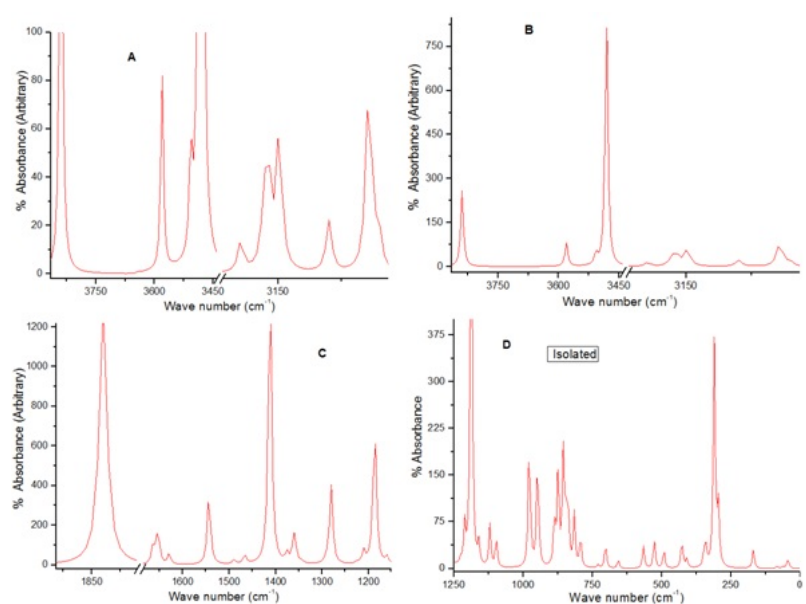

Figure 4(a): Computed IR spectrum of the isolated molecule of L-TYR.
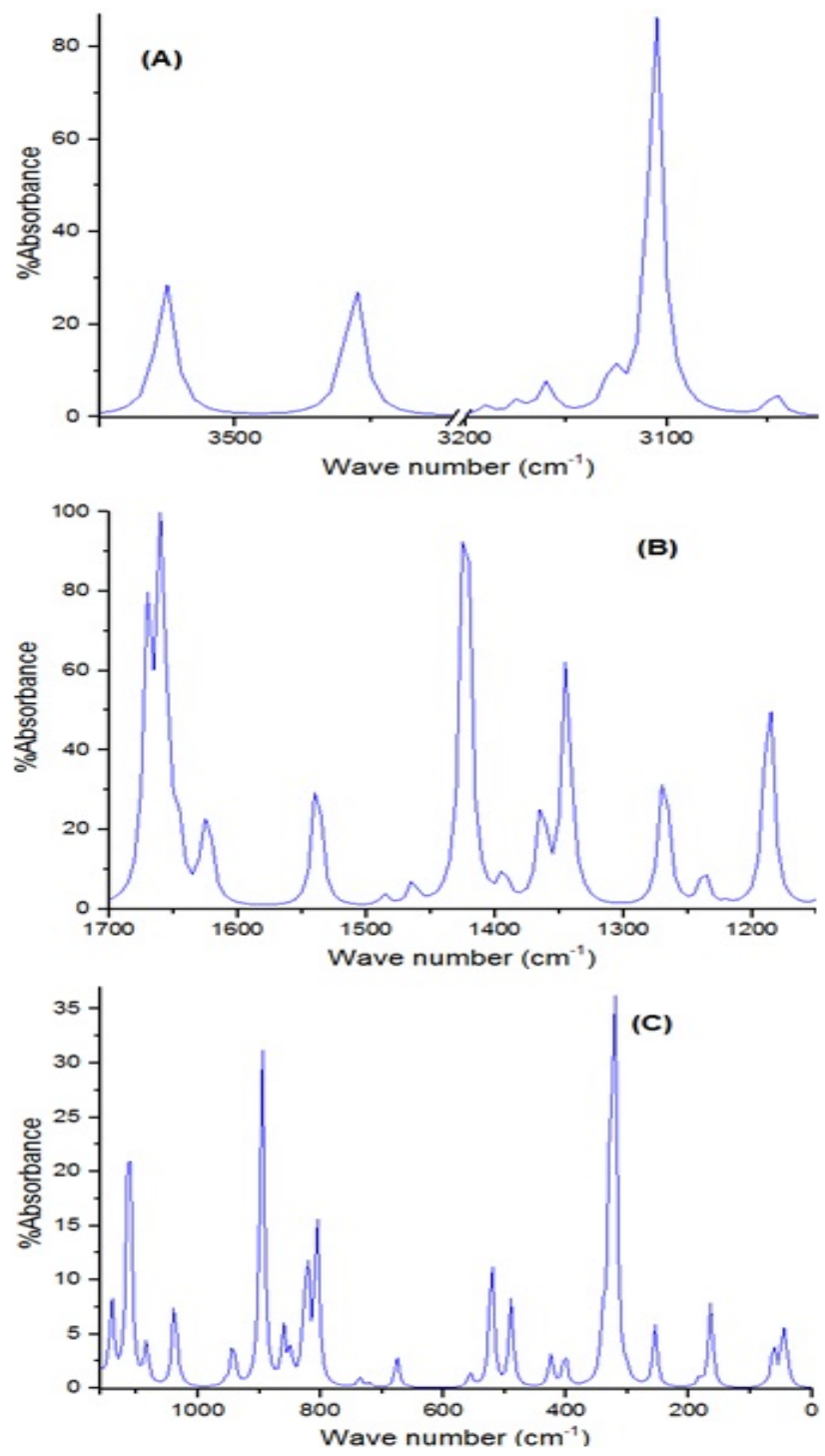

Figure 4(b): Computed IR spectrum of the zwitterion of L-TYR. 
Citation: Yadav RA, Dixit V, Yogesh M, Santhosh C (2015) Raman and IR Spectral and DFT Based Vibrational and Electronic Characterization of Isolated and Zwitterionic Forms of L-Tyrosine. Pharm Anal Acta 6: 439. doi:10.4172/21532435.1000439
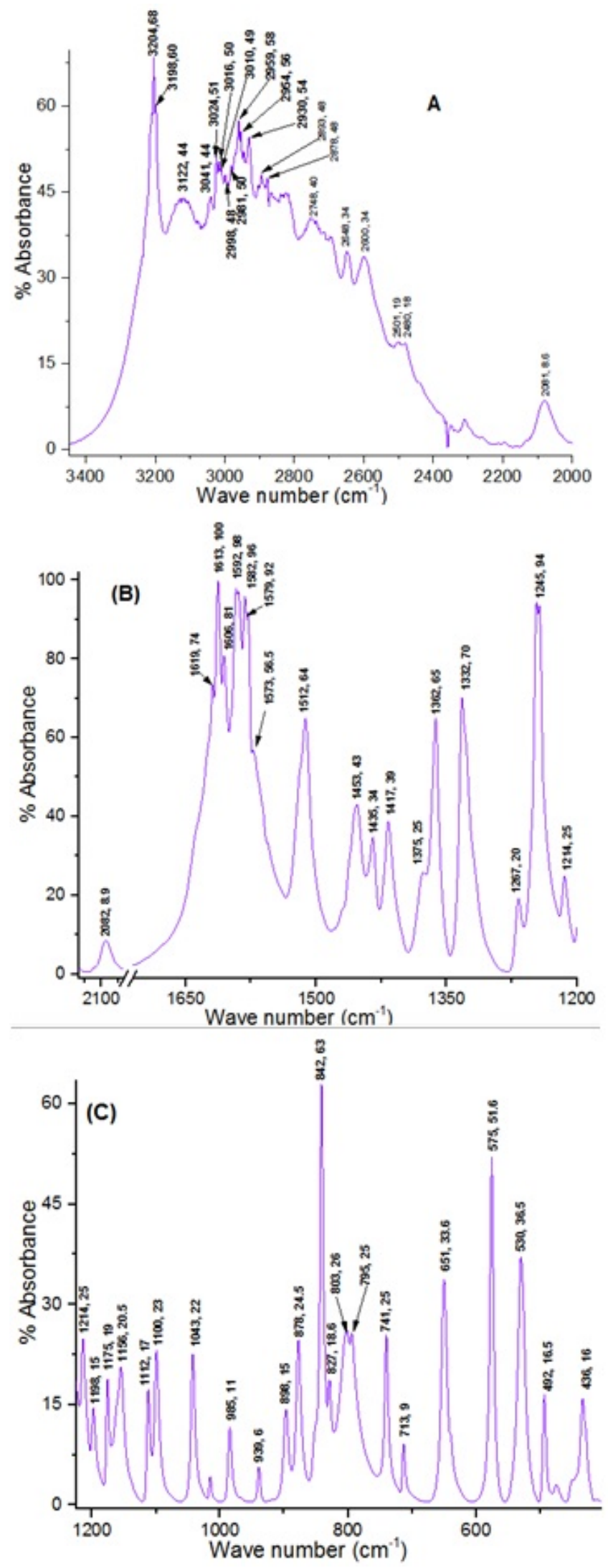

Figure 5: Experimentally observed IR spectrum of the L-TYR.

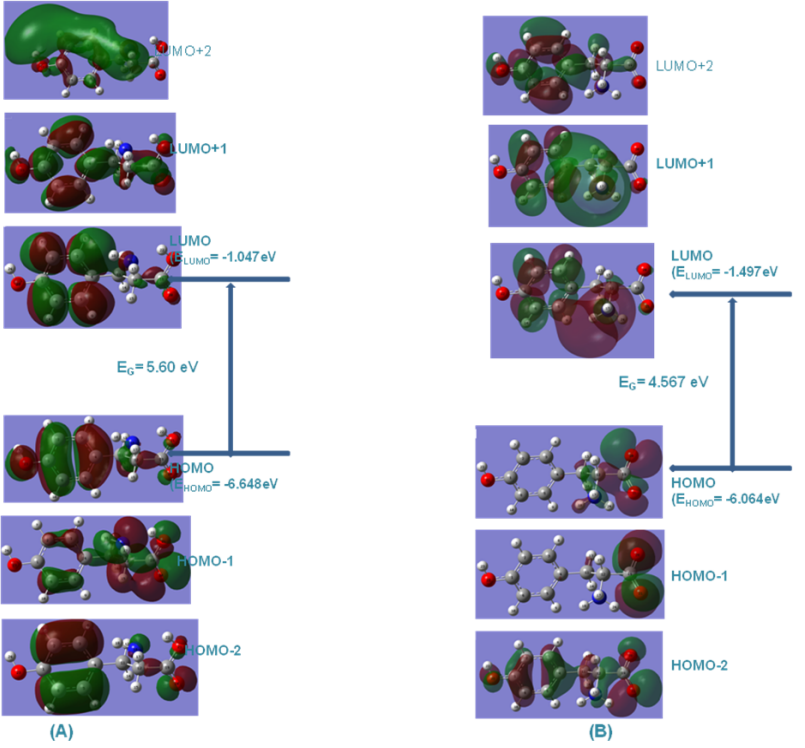

Figure 6: The atomic orbital compositions of the frontier $\mathrm{MO}$ of $(\mathrm{A})$ Isolated species and (B) Zwitterion of the L-Tyrosine.

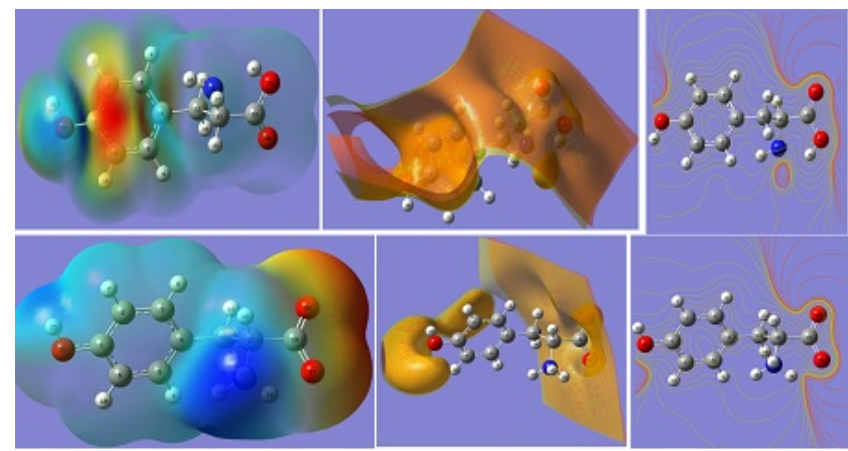

Figure 7: Total density mappings, MEP mappings and MEP arrays of isolated species (upper) and zwitterion of L-TYR.

LTRYP; at 1722, 1661, 1640, 1517, 1396 and $937 \mathrm{~cm}^{-1}$ by Contreras et al. [6] in zwitterionic L-Tyrosine (L-TYR) and at 1565, 1546, 1455, 1269, 1052 and $1000 \mathrm{~cm}^{-1}$ by Dixit et al. [36] in zwitterionic L-Phenylalanine (LPHAL) respectively. These modes are generally coupled with the other modes. For isolated molecule, the ring planar deformation modes $\alpha_{1}(R), \alpha_{3}(R)$ and $\alpha_{2}(R)$ have been computed to be $983\left(v_{34}\right), 641\left(v_{49}\right)$ and $481\left(v_{52}\right) \mathrm{cm}^{-1}$ while in zwitterionic form they correspond to the frequencies $981\left(v_{36}\right), 640\left(v_{49}\right)$ and $480\left(v_{52}\right) \mathrm{cm}^{-1}$, corresponding to the frequencies 876, 552 and $445 \mathrm{~cm}^{-1}$ in zwitterionic L-TRYP [Layton et al. Ref. [35]]; 949, 606 and $361 \mathrm{~cm}^{-1}$ in zwitterionic L-TYR [Contreras et al. Ref. [6]] and also 968,620 and $572 \mathrm{~cm}^{-1}$ in zwitterionic L-PHAL [Dixit et al. Ref. [36]]; all of these modes are coupled with the other modes. The three ring non-planar deformation modes $\Phi_{1}(\mathrm{R}), \Phi_{2}(\mathrm{R})$ and $\Phi_{3}(\mathrm{R})$, which were assigned at the frequencies 742,569 and 420 $\mathrm{cm}^{-1}$ by Layton et al. [35] in zwitterionic L-TRYP; at 659, 490 and 402 $\mathrm{cm}^{-1}$ by Contreras et al. [6] in zwitterionic L-TYR and at 749, 406 and $232 \mathrm{~cm}^{-1}$ by Dixit et al. [36] in zwitterionic L-PHAL respectively, are correlated to the frequencies $714\left(v_{46}\right), 515\left(v_{51}\right)$ and $414\left(v_{54}\right) \mathrm{cm}^{-1}$ in isolated species while in zwitterionic form they have been correlated to 
Citation: Yadav RA, Dixit V, Yogesh M, Santhosh C (2015) Raman and IR Spectral and DFT Based Vibrational and Electronic Characterization of Isolated and Zwitterionic Forms of L-Tyrosine. Pharm Anal Acta 6: 439. doi:10.4172/21532435.1000439

the frequencies $721\left(v_{46}\right), 415\left(v_{54}\right)$ and $160\left(v_{62}\right) \mathrm{cm}^{-1}$ corresponding to modes 4 and $16(\mathrm{a}, \mathrm{b})$ of the benzene molecule. These have been found to couple with many deformations, however few $\alpha(R)$ and $\Phi(R)$ modes are pure and localized.

The $\mathrm{CH}$ stretching modes $v\left(\mathrm{C}_{2}-\mathrm{H}_{7}\right),\left(\mathrm{C}_{5}-\mathrm{H}_{9}\right), v\left(\mathrm{C}_{3}-\mathrm{H}_{8}\right)$ and $v\left(\mathrm{C}_{6}-\mathrm{H}_{10}\right)$ have been assigned at the frequencies $3007\left(v_{8}\right), 3018\left(v_{7}\right)$, $3024\left(v_{6}\right)$ and $3050\left(v_{5}\right) \mathrm{cm}^{-1}$ respectively in isolated form and at the frequencies $3017\left(v_{7}\right), 3019\left(v_{6}\right), 3031\left(v_{5}\right)$ and $3047\left(v_{4}\right) \mathrm{cm}^{-1}$ respectively in zwitterionic form. The $v(\mathrm{C}-\mathrm{H})$ modes were assigned at 3057,3045 , 3035 and $3024 \mathrm{~cm}^{-1}$ by Layton et al. [35] in zwitterionic L-TRYP; at 2941, 2940, 2939 and $2939 \mathrm{~cm}^{-1}$ by Contreras et al. [6] in zwitterionic L-TYR and also at 3048, 3037, 3029 and $3018 \mathrm{~cm}^{-1}$ by Dixit et al.[36] in zwitterionic L-PHAL respectively. The $\mathrm{C}-\mathrm{H}$ stretching vibrations are pure and highly localized modes, however, they are found to occur in association with one another. The $v\left(\mathrm{C}_{1}-\mathrm{O}_{23}\right)$ and $v\left(\mathrm{C}_{4}-\mathrm{C}_{12}\right)$ modes occur at frequencies $1222\left(v_{26}\right)$ and $687\left(v_{47}\right) \mathrm{cm}^{-1}$ in isolated molecule while in zwitterion they have been assigned at the frequencies $1268\left(v_{27}\right)$ and $706\left(v_{47}\right) \mathrm{cm}^{-1}$, these modes are also coupled with many other modes.

The $\mathrm{CH}$ planar deformation modes $\beta\left(\mathrm{C}_{6}-\mathrm{H}_{10}\right), \beta\left(\mathrm{C}_{3}-\mathrm{H}_{8}\right), \beta\left(\mathrm{C}_{5}-\mathrm{H}_{9}\right)$ and $\beta\left(\mathrm{C}_{2}-\mathrm{H}_{7}\right)$ have been found to correspond to the frequencies $1070\left(v_{32}\right), 1141\left(v_{29}\right) 1300\left(v_{21}\right)$ and $1474\left(v_{16}\right) \mathrm{cm}^{-1}$ respectively in isolated molecule while to the frequencies $1090\left(v_{32}\right), 1140\left(v_{30}\right) 1303\left(v_{22}\right)$ and $1469\left(v_{17}\right) \mathrm{cm}^{-1}$ respectively in zwitterionic molecule. The frequencies, $1531,1454,1441$ and $1275 \mathrm{~cm}^{-1}$, were assigned to the $\mathrm{CH}$ planar deformation modes in zwitterionic form of L-TRYP by Layton et al. [35]. For zwitterionic L-TYR only three frequencies 1213, 1069 and $1051 \mathrm{~cm}^{-1}$ were assigned to the $\mathrm{CH}$ planar deformation modes by Contreras et al. [6], which seems to be incomplete assignment. The frequencies 1412,1304, 1290, 1145 and $1124 \mathrm{~cm}^{-1}$, were assigned to the $\mathrm{CH}$ planar deformation modes by Dixit et al [36]. in zwitterionic L-PHAL respectively. These modes appear in strong association with other many modes. The $\mathrm{CH}$ non-planar deformation modes $\gamma\left(\mathrm{C}_{5}-\mathrm{H}_{9}\right)$, $\gamma\left(\mathrm{C}_{3}-\mathrm{H}_{8}\right), \gamma\left(\mathrm{C}_{6}-\mathrm{H}_{10}\right)$ and $\gamma\left(\mathrm{C}_{2}-\mathrm{H}_{7}\right)$ have been found dominantly in the frequencies $952\left(v_{36}\right), 922\left(v_{38}\right), 819\left(v_{43}\right)$ and $798\left(v_{44}\right) \mathrm{cm}^{-1}$ respectively in isolated form of molecule while in zwitterion these have been observed at the frequencies $958\left(v_{37}\right), 936\left(v_{38}\right), 812\left(v_{43}\right)$ and $804\left(v_{44}\right)$ $\mathrm{cm}^{-1}$ respectively corresponding to the frequencies $965,916,854$ and $753 \mathrm{~cm}^{-1}$ by Layton et al. [35] in zwitterionic L-TRYP; 1122, 1108, 902 and $891 \mathrm{~cm}^{-1}$ by Contreras et al. [6] in zwitterionic L-TYR and 966 , 950, 894, 844 and $700 \mathrm{~cm}^{-1}$ by Dixit et al. [36] in zwitterionic L-PHAL respectively. The modes $\beta\left(\mathrm{C}_{4}-\mathrm{C}_{11}\right), \gamma\left(\mathrm{C}_{4}-\mathrm{C}_{11}\right), \beta\left(\mathrm{C}_{1}-\mathrm{O}_{23}\right)$ and $\gamma\left(\mathrm{C}_{1}-\mathrm{O}_{23}\right)$ correspond to the frequencies $300\left(v_{59}\right), 62\left(v_{64}\right), 419\left(v_{53}\right)$ and $340\left(v_{56}\right)$ $\mathrm{cm}^{-1}$ respectively in isolated molecule while these modes have been calculated to be $296\left(v_{59}\right), 545\left(v_{50}\right), 417\left(v_{53}\right)$ and $511\left(v_{51}\right) \mathrm{cm}^{-1}$ respectively in zwitterion, which are also coupled with other modes.

OH group modes: The $\mathrm{OH}$ group attached directly to the phenyl ring at the site $\mathrm{C}_{1}$ is associated with three normal modes of the vibrations; $\mathrm{OH}$ stretching mode $-v\left(\mathrm{O}_{23}-\mathrm{H}_{24}\right), \mathrm{COH}$ angle bending mode $-\mathrm{a}\left(\mathrm{C}_{1}-\mathrm{O}_{22}-\mathrm{H}_{23}\right)$ and $\mathrm{OH}$ torsion mode $-\tau\left(\mathrm{C}_{1}-\mathrm{O}_{22}\right)$ which have been assigned in the present case for isolated molecule at the frequencies $3662\left(v_{1}\right), 1134\left(v_{30}\right)$ and $303\left(v_{58}\right) \mathrm{cm}^{-1}$ and for the zwitterionic form at $3640\left(v_{1}\right), 1134\left(v_{31}\right)$ and $314\left(v_{58}\right) \mathrm{cm}^{-1}$ respectively, corresponding to the earlier assigned frequencies 3557,943 and $290 \mathrm{~cm}^{-1}[6]$ respectively. The stretching and torsion modes are pure and highly localized while bending mode occurs in association with the other modes.

Carbon chain modes: The side carbon chain attached to the $\mathrm{C}_{4}$ atom of the phenyl ring (Figure 1 ) is associated with 33 normal modes of vibration. The twisting modes $\tau\left(\mathrm{C}_{4}-\mathrm{C}_{12}\right), \tau\left(\mathrm{C}_{12}-\mathrm{C}_{13}\right)$, corresponding to the frequencies 31 and $66 \mathrm{~cm}^{-1}$ [35] in zwitterionic L-TRYP; 28 and 87 $\mathrm{cm}^{-1}$ [6] in zwitterionic L-TYR and 33 and $49 \mathrm{~cm}^{-1}$ [36] in zwitterionic L-PHAL, have been found in isolate and zwitterion at the frequencies $39\left(v_{66}\right), 45\left(v_{65}\right) \mathrm{cm}^{-1}$ and $39\left(v_{66}\right), 46\left(v_{65}\right) \mathrm{cm}^{-1}$. The angle bending modes $\alpha\left(\mathrm{C}_{11}-\mathrm{C}_{12}-\mathrm{C}_{19}\right), \alpha\left(\mathrm{C}_{4}-\mathrm{C}_{11}-\mathrm{C}_{12}\right), \alpha\left(\mathrm{N}_{16}-\mathrm{C}_{12}-\mathrm{C}_{19}\right), \alpha\left(\mathrm{N}_{16}-\mathrm{C}_{12}-\mathrm{C}_{11}\right), \alpha\left(\mathrm{N}_{16}-\right.$ $\left.\mathrm{C}_{12}-\mathrm{H}_{15}\right)$ and $a\left(\mathrm{C}_{11}-\mathrm{C}_{12}-\mathrm{H}_{15}\right)$ correspond to the frequencies $165\left(v_{62}\right)$, $182\left(v_{61}\right), 333\left(v_{57}\right), 400\left(v_{55}\right), 1297\left(v_{22}\right)$ and $1314\left(v_{20}\right) \mathrm{cm}^{-1}$ respectively for isolated species while they have been found for zwitterion at the frequencies $179\left(v_{61}\right), 61\left(v_{64}\right), 322\left(v_{57}\right), 393\left(v_{55}\right), 1218\left(v_{26}\right)$ and $1330\left(v_{21}\right)$ $\mathrm{cm}^{-1}$ respectively. The stretching modes $v\left(\mathrm{C}_{12}-\mathrm{C}_{19}\right), v\left(\mathrm{C}_{11}-\mathrm{C}_{12}\right), v\left(\mathrm{C}_{12}-\mathrm{N}_{16}\right)$ and $v\left(\mathrm{C}_{12}-\mathrm{H}_{15}\right)$ have been computed to be $928\left(v_{37}\right), 1048\left(v_{33}\right), 855\left(v_{40}\right)$ and $2896\left(v_{11}\right) \mathrm{cm}^{-1}$ and $877\left(v_{40}\right), 991\left(v_{35}\right), 842\left(v_{41}\right)$ and $2986\left(v_{8}\right) \mathrm{cm}^{-1}$ respectively for isolated and zwitterion L-TYR molecule. All of these modes, except $v\left(\mathrm{C}_{12}-\mathrm{H}_{15}\right)$, have been found to couple with a number of vibrational modes. The $v\left(\mathrm{C}_{12}-\mathrm{H}_{15}\right)$ mode have been found localized and pure. These stretching modes were assigned by Layton et al. [35] in zwitterionic L-TRYP at the frequencies 582, 988, 770 and $2942 \mathrm{~cm}$ ${ }^{1}$; Contreras et al. [6] assigned these modes in zwitterionic L-TYR at the frequencies $823,1140,1024$ and $2826 \mathrm{~cm}^{-1}$ while for zwitterionic L-PHAL Dixit et al.[36] assigned these modes at 875, 992, 809 and 2983 $\mathrm{cm}^{-1}$.

6.3.3.1 $\mathrm{COOH} / \mathrm{CO}_{2}$ group modes: In the isolated L-TYR molecule, the $\mathrm{COOH}$ group is associated with the stretching modes $v\left(\mathrm{O}_{21}-\mathrm{H}_{22}\right)$, $v\left(\mathrm{C}_{19}-\mathrm{O}_{20}\right)$ and $v\left(\mathrm{C}_{19}-\mathrm{O}_{21}\right)$ which have been assigned at the frequencies $3324\left(v_{4}\right), 1752\left(v_{12}\right)$ and $1156\left(v_{28}\right) \mathrm{cm}^{-1}$, which were found at 3302, 1800 and $862 \mathrm{~cm}^{-1}$ by Govindarasu et al. [31], respectively while the anti-symmetric and symmetric stretching of $\mathrm{CO}_{2}$ group in zwitterion, $v_{\mathrm{as}}\left(\mathrm{CO}_{2}\right)$ and $v_{\mathrm{s}}\left(\mathrm{CO}_{2}\right)$, have been assigned at the frequencies 1584 $\left(v_{13}\right)$ and $1284\left(v_{24}\right) \mathrm{cm}^{-1}$, which were already found corresponding to the frequencies 1688 and $1290 \mathrm{~cm}^{-1}$ by Layton et al. [35] in zwitterionic L-TRYP; at 1757 and $1557 \mathrm{~cm}^{-1}$ by Contreras et al. [6] in zwitterionic L-TYR and also at 1594 and $1283 \mathrm{~cm}^{-1}$ by Dixit et al. [36] in zwitterionic L-PHAL. The $\mathrm{OH}$ stretching mode is highly localised and pure but the later are coupled with other modes. The angle bending mode $a\left(\mathrm{C}_{19}{ }^{-}\right.$ $\left.\mathrm{O}_{21}-\mathrm{H}_{22}\right)$ and the $\mathrm{OH}$ torsion mode $\tau\left(\mathrm{C}_{19}-\mathrm{O}_{21}\right)$ of $\mathrm{COOH}$ Group have been assigned at the frequencies $1348\left(v_{19}\right)$ and $837\left(v_{41}\right) \mathrm{cm}^{-1}$. These modes were found already by Govindarasu et al. [31] at 1387 and $862 \mathrm{~cm}^{-1}$. The first one is highly localised while the later is coupled with other modes. The modes $\tau\left(\mathrm{C}_{12}-\mathrm{C}_{19}\right), \omega(\mathrm{COOH}), \rho(\mathrm{COOH})$ and $\delta(\mathrm{COOH})$ have been assigned at the frequencies $81\left(v_{63}\right), 775\left(v_{45}\right)$, 553 $\left(v_{50}\right)$ and $643\left(v_{48}\right) \mathrm{cm}^{-1}$ respectively in isolated molecule while the modes $\tau\left(\mathrm{C}_{12}-\mathrm{C}_{19}\right), \omega\left(\mathrm{CO}_{2}\right), \rho\left(\mathrm{CO}_{2}\right)$ and $\delta\left(\mathrm{CO}_{2}\right)$ have been assigned at the frequencies $75\left(v_{63}\right), 662\left(v_{48}\right), 334\left(v_{55}\right)$ and $789\left(v_{45}\right) \mathrm{cm}^{-1}$ respectively in zwitterion, all of these have been found to be coupled with other modes. These modes had already been assigned at the frequencies 61, 847,503 and $819 \mathrm{~cm}^{-1}$ by Layton et al. [35]; at 36, 677, 452 and $609 \mathrm{~cm}^{-1}$ by Contreras et al. [6] in zwitterionic L-TYR and also at 65, 662, 506 and $735 \mathrm{~cm}^{-1}$ by Dixit et al. [36] in zwitterionic L-PHAL respectively.

$\mathbf{N H}_{2} / \mathbf{N H}_{3}$ group modes: In isolated L-TYR molecule there are six modes of vibration due to the $\mathrm{NH}_{2}$ group, namely, anti-symmetric $\mathrm{NH}_{2}$ stretching $-v_{2 s}\left(\mathrm{NH}_{2}\right)$, symmetric $\mathrm{NH}_{2}$ stretching $-v_{\mathrm{s}}\left(\mathrm{NH}_{2}\right), \mathrm{NH}_{2}$ scissoring $-\delta\left(\mathrm{NH}_{2}\right), \mathrm{NH}_{2}$ rocking $-\rho\left(\mathrm{NH}_{2}\right), \mathrm{NH}_{2}$ wagging $-\omega\left(\mathrm{NH}_{2}\right)$ and $\mathrm{NH}_{2}$ torsion- $\tau\left(\mathrm{NH}_{2}\right)$ corresponding to the frequencies $3415\left(v_{2}\right)$, $3349\left(v_{3}\right), 1588\left(v_{13}\right), 1108\left(v_{31}\right), 868\left(v_{39}\right)$ and $290\left(v_{60}\right) \mathrm{cm}^{-1}$ respectively. The modes $v_{\mathrm{as}}\left(\mathrm{NH}_{2}\right), v_{\mathrm{s}}\left(\mathrm{NH}_{2}\right), \delta\left(\mathrm{NH}_{2}\right)$, and $\tau\left(\mathrm{NH}_{2}\right)$ were assigned at $3447,3370,1605$ and $290 \mathrm{~cm}^{-1}$ by Govindarasu et al. [31] for the isolated L-PHAL molecule. The anti-symmetric $\mathrm{NH}_{2}$ stretching $-v_{\text {as }}\left(\mathrm{NH}_{2}\right), \mathrm{NH}_{2}$ scissoring $-\delta\left(\mathrm{NH}_{2}\right)$ are pure and highly localized modes; the symmetric $\mathrm{NH}_{2}$ stretching- $v_{s}\left(\mathrm{NH}_{2}\right)$ is slightly coupled with the $v\left(\mathrm{O}_{21}-\mathrm{H}_{22}\right)$ mode in isolated state, the $\mathrm{NH}_{2}$ torsion- $\tau\left(\mathrm{NH}_{2}\right)$ is slightly coupled with the $\tau\left(\mathrm{C}_{19}-\mathrm{O}_{21}\right)$ and $\beta\left(\mathrm{C}_{4}-\mathrm{C}_{11}\right), \mathrm{NH}_{2}$ rocking $-\rho\left(\mathrm{NH}_{2}\right)$ is slightly coupled with 
Citation: Yadav RA, Dixit V, Yogesh M, Santhosh C (2015) Raman and IR Spectral and DFT Based Vibrational and Electronic Characterization of Isolated and Zwitterionic Forms of L-Tyrosine. Pharm Anal Acta 6: 439. doi:10.4172/21532435.1000439

$\omega(\mathrm{COOH})$ and $\Phi_{1}(\mathrm{R})$ while $\mathrm{NH}_{2}$ wagging $-\omega\left(\mathrm{NH}_{2}\right)$ is coupled with $\beta\left(\mathrm{C}_{12}-\mathrm{C}_{19}\right)$

For the zwitterionic L-TYR molecule the nine modes of vibration due to the $\mathrm{NH}_{3}$ group as seen from the Gauss View software are: an anti-symmetric $\mathrm{NH}_{2}$ stretching $-v_{\mathrm{as}}\left(\mathrm{NH}_{2}\right)$, a symmetric $\mathrm{NH}_{2}$ stretching $-v_{\mathrm{s}}\left(\mathrm{NH}_{2}\right)$, an $\mathrm{NH}$ stretching $-v(\mathrm{NH})$, two anti-symmetric $\mathrm{NH}_{3}$ deformations $-\delta_{a s}\left(\mathrm{NH}_{3}\right)$, the symmetric $\mathrm{NH}_{3}$ deformation $\delta_{s}\left(\mathrm{NH}_{3}\right)$, the planar $\mathrm{NH}_{3}$ rocking $-\rho_{2}\left(\mathrm{NH}_{3}\right)$, the perpendicular $\mathrm{NH}_{3}$ rocking $-\rho_{1}\left(\mathrm{NH}_{3}\right)$ and the $\mathrm{NH}_{3}$ torsion- $\tau\left(\mathrm{NH}_{2}\right)$ which correspond to the calculated frequencies $3367\left(v_{2}\right), 3300\left(v_{3}\right), 2911\left(v_{9}\right), 1594\left(v_{12}\right)$, $1549\left(v_{16}\right), 1359\left(v_{20}\right), 1062\left(v_{33}\right), 1035\left(v_{34}\right)$ and $250\left(v_{60}\right) \mathrm{cm}^{-1}$ respectively. These modes had been assigned at 3295, 3228, 2918, 1623, 1561, 1387, 1105,1070 and $329 \mathrm{~cm}^{-1}$ by Layton et. al. [30] in zwitterionic L-TRYP; at $3204,3096,3204,1459,1456,1491,1104,1101$ and $206 \mathrm{~cm}^{-1}$ by Contreras et al. [6] in zwitterionic L-TYR and also at 3366, 3301, 2949, 1585, 1551, 1358, 1030, 900 and $254 \mathrm{~cm}^{-1}$ by Dixit et al. [31] in zwitterionic L-PHAL respectively. The anti-symmetric $\mathrm{NH}_{2}$ stretching $-v_{\text {as }}\left(\mathrm{NH}_{2}\right)$, symmetric $\mathrm{NH}_{2}$ stretching $-v_{s}\left(\mathrm{NH}_{2}\right)$, $\mathrm{NH}$ stretching $-v(\mathrm{NH})$, $\mathrm{NH}_{2}$ scissoring $-\delta\left(\mathrm{NH}_{2}\right)$ are pure and highly localized modes. The $\mathrm{NH}_{3}$ torsion- $\tau\left(\mathrm{NH}_{3}\right)$ is slightly coupled with the $\tau\left(\mathrm{C}_{19}-\mathrm{O}_{21}\right)$ and $\beta\left(\mathrm{C}_{4}-\mathrm{C}_{11}\right)$.

\section{$\mathrm{CH}_{2}$ group modes}

The six modes of vibrations due to the $\mathrm{CH}_{2}$ group are assigned at the frequencies $2951\left(v_{9}\right), 2906\left(v_{10}\right), 1422\left(v_{17}\right), 1234\left(v_{24}\right), 1230\left(v_{25}\right)$ and $958\left(v_{35}\right) \mathrm{cm}^{-1}$ respectively in the isolated molecule while they have been assigned at the frequencies 2954( $\left.v_{10}\right), 2910\left(v_{11}\right), 1419\left(v_{18}\right)$, $1272\left(v_{25}\right), 1181\left(v_{28}\right)$ and $923\left(v_{39}\right) \mathrm{cm}^{-1}$ respectively in the zwitterionic form. These modes were assigned at the frequencies 2989, 2927, 1436, 1337, 1369 and $906 \mathrm{~cm}^{-1}$ by Layton et al. [30] in zwitterionic L-TRYP; at 2856, 2793, 1400, 1590, 1256 and $795 \mathrm{~cm}^{-1}$ by Contreras et al. [6] in zwitterionic L-TYR and at 2957, 2911, 1420, 1332, 1086 and $828 \mathrm{~cm}^{-1}$ by Dixit et al. [31] in zwitterionic L-PHAL respectively. The $v_{\text {as }}\left(\mathrm{CH}_{2}\right)$, $v_{s}\left(\mathrm{CH}_{2}\right)$ and $\delta\left(\mathrm{CH}_{2}\right)$ are pure and highly localized modes while $v_{\mathrm{s}}\left(\mathrm{CH}_{2}\right)$ in isolated molecule is coupled with $v\left(\mathrm{C}_{12}-\mathrm{H}_{15}\right)$ and the other modes are strongly coupled with various modes.

\section{HOMO-LUMO energy gap}

The HOMO and LUMO are the principal orbitals which are essential in predicting the chemical stability of a molecule $[32,36]$. The kinetic stability of the molecules is generally expressed in terms of the forbidden energy gap $\mathrm{E}_{\mathrm{g}}[33,34]$. The energy gap of the HOMO and LUMO is a measure of the reactivity of the molecule. The smaller the energy gap more easily it can be excited and vice versa. The smaller HOMO-LUMO energy gap stimulates the eventual ICT interaction taking place within the molecule, which is responsible for the bioactivity of the molecule. The larger value of the energy gap is responsible for the higher kinetic stability and lower chemical reactivity because it would be energetically more difficult to add the electrons to the LUMO and to remove the electrons from the HOMO [35,36]. The schematic diagram of the atomic orbital compositions of the frontier MOs have been shown for isolated and also for the zwitterionic L-TYR molecule in Figure 6. The green and red coloured solid regions in the Figure 6 exhibit the MOs with the completely opposite phases. The present calculations predict the energy gap $\left(\mathrm{E}_{\mathrm{g}}\right)$, i.e. the transition energy from HOMO to LUMO of the isolated and zwitterionic L-TYR molecule to be $5.60 \mathrm{eV}$ and $4.567 \mathrm{eV}$.

The Molecule is found to possess a dipole moment 6.11 Debye for isolated state and 12.99 Debye for zwitterionic form, the experimental value of $\mu=13.95 \mathrm{D}$ [6] is in agreement with that of lowest energy conformer of zwitterionic form. The electric polarizability components $\alpha_{x x}, \alpha_{x y}, \alpha_{y y}, \alpha_{x z}, \alpha_{y z}$ and $\alpha_{z z}$ for isolated molecule and zwitterion have been computed as 166.23, -4.68, 120.61, 8.44, 7.08 and $88.00 \mathrm{Bohr}^{3}$ and $211.73,5.52,167.90,-15.54,12.56$ and $124.61 \mathrm{Bohr}^{3}$, respectively resulting in the net isotropic polarizability $124.94 \mathrm{Bohr}^{3}$ and 168.08 Bohr $^{3}$. It is noticeable that both the essential parameters electric dipole moment and electric polarizability significantly higher in the zwitterionic form.

\section{Molecular electrostatic potentials}

The molecular electrostatic potentials (MEP) are the essential tools to analyse the inter-molecular association and also the molecular properties of small molecules, actions of the molecules of the drugs and their analogues, the biological functioning of the haemoglobin and enzyme catalysis [35-39]. These are widely utilized as the reactivity map displaying the most probable regions for the electrophilic attack of the point-like charged reagents on the organic molecules [40]. The values and the spatial distribution of the MEPs are generally responsible for the chemical nature of an agent in the chemical reaction. They strongly affect the binding of the substrate to its active site. These potentials would be typically visualized through mapping its values onto the molecular ED. The different values of the MEPs at the surface are represented by different colours; the red colour represents region of the most negative electrostatic potential, the blue colour represents region of the most positive electrostatic potential and the green represents region of zero potential. Potentials occur in the order (MEP)red < (MEP)orange < (MEP)yellow < (MEP)green < (MEP)blue. While the negative electrostatic potential is corresponding to the proton attraction by the concentrated ED in the molecule (and is coloured in shades of red), the positive electrostatic potential is corresponding to the proton repulsion by atomic nuclei in regions where a low ED exists and therefore, the nuclear charge is incompletely shielded (and is coloured in shades of blue). The total density plots and corresponding arrays as well as the MEPs mappings and corresponding arrays for both the considered forms of the L-TYR molecule have been shown in Figure 7. These figures provide a visual demonstration of the chemically active sites and the comparative reactivity of atoms in the molecule. A close investigation of these mappings and arrays predicted that in isolated form of L-TYR the regions nearby each of the functional group, namely, $\mathrm{NH}_{2}, \mathrm{COOH}$ and $\mathrm{OH}$ groups, provide an active site for neucleo-philic attack, while in zwitterion $\mathrm{CO}_{2}$ group provide a vast region and $\mathrm{OH}$ group provide one site for neucleo-philic attack but no active region has been provided by the $\mathrm{NH}_{3}$ group.

\section{Analysis on natural orbital bonding}

In order to evaluate the electron density distribution amongst the atoms and bonds at the various sites, natural (localized) orbitals are used in computational chemistry. In the localized 1-centre and 2-centre regions of the molecule these generally represent the "maximum occupancy character". The NBOs include the highest possible percentage of the ED, which is ideally close to 2.000 , providing the most accurate possible "natural Lewis structure" of the Schrodinger wave function $\psi$. The high percentage of the ED, often found to be $>99 \%$ for the common organic molecules, corresponds to an accurate natural Lewis structure.

NBO Interactions: Second order perturbation theory : The natural bond orbitals (NBO) investigation is essential to explain the second-order interactions between the occupied NBOs of one subsystem and unoccupied NBOs of another subsystem, which would be a measure of the intermolecular delocalization or hyper conjugation 
Citation: Yadav RA, Dixit V, Yogesh M, Santhosh C (2015) Raman and IR Spectral and DFT Based Vibrational and Electronic Characterization of Isolated and Zwitterionic Forms of L-Tyrosine. Pharm Anal Acta 6: 439. doi:10.4172/21532435.1000439

[41-46]. The delocalization interaction strengths, $\mathrm{E}(2)$, estimated by the second order perturbation theory [31] is given by ,

$$
E(2)=\Delta E_{i j}=q_{i} \frac{F_{i j}^{2}}{a_{j}-\check{a}_{i}}
$$

where $\mathrm{q}_{\mathrm{i}}, \mathrm{E}_{\mathrm{i}}, \mathrm{E}_{\mathrm{j}}$ and $\mathrm{F}_{\mathrm{ij}}$ are donor occupancy, diagonal elements and off diagonal NBO Fock matrix elements.

The interaction energy values between the occupied (donors) i and unoccupied (acceptors) $\mathrm{j}$ NBOs, calculated using the second order perturbation theory have been collected in Table 7, Table 8 and Table 9 for isolated and zwitterionic LTYR molecule. The larger value of the interaction energy of the NBOs $\mathrm{E}(2)$ signifies more intense interaction between donors and acceptors, i.e. higher the electrons donating tendency from donor to acceptor NBOs. The energy values involved in the hyperconjucative interactions between the carbon atoms in the phenyl ring $\pi\left(\mathrm{C}_{1}-\mathrm{C}_{2}\right) \pi^{*}\left(\mathrm{C}_{3}-\mathrm{C}_{4}\right)$ and $\pi^{\star}\left(\mathrm{C}_{5}-\mathrm{C}_{6}\right)$, and $\pi\left(\mathrm{C}_{3}-\mathrm{C}_{4}\right)$ $\rightarrow \pi^{\star}\left(\mathrm{C}_{1}-\mathrm{C}_{2}\right)$ and $\pi^{\star}\left(\mathrm{C}_{5}-\mathrm{C}_{6}\right), \pi\left(\mathrm{C}_{5}-\mathrm{C}_{6}\right) \rightarrow \pi^{\star}\left(\mathrm{C}_{1}-\mathrm{C}_{2}\right)$ and $\pi^{\star}\left(\mathrm{C}_{3}\right.$ $-\mathrm{C}_{4}$ ) are 21.63 and $16.56,17.85$ and $20.93,22.07$ and $18.33 \mathrm{kcal} / \mathrm{mole}$ respectively (Table-7) for isolated system while in the zwitterionic state the hyperconjucative binding energy values for the phenyl ring $\mathrm{C}$ atoms $\pi\left(\mathrm{C}_{2}-\mathrm{C}_{3}\right) \mathrm{LP}^{*}(1) \mathrm{C}_{1}$ and $\mathrm{LP}(1) \mathrm{C}_{4}, \pi^{*}\left(\mathrm{C}_{5}-\mathrm{C}_{6}\right) \mathrm{LP}^{*}(1) \mathrm{C}_{1}$ and $\mathrm{LP}(1) \mathrm{C}_{4}$ have been computed $53.69,41.08,54.09$ and $44.40 \mathrm{kcal} / \mathrm{mole}$ respectively (Table-8). The hyper conjugative interaction and the $\mathrm{E}_{\mathrm{D}}$ transfer from the lone pair of the $\mathrm{N}_{16}$ atom to the $\mathrm{O}_{21}-\mathrm{H}_{22}$ orbital in the $\mathrm{O}_{21}-\mathrm{H}_{22}---$ $-\mathrm{N}_{16}$ system is found to be present with the energy $10.34 \mathrm{kcal} / \mathrm{mole}$ in the isolated molecule while the interaction and ED transfer from the lone pair of the $\mathrm{O}_{21}$ atom to $\mathrm{N}_{16}-\mathrm{H}_{24}$ orbital in the $\mathrm{N}_{16}-\mathrm{H}_{24} \ldots \ldots . \mathrm{O}_{21}$ system appears to be present with the interaction energy $14.22 \mathrm{kcal} / \mathrm{mole}$ in zwitterion. Obviously, stronger intramolecular $\mathrm{H}$ bonding exists in the zwitterionic system than isolated system. The intramolecular hydrogen bonding would be possible by the orbital overlapping between LP (1) $\mathrm{N}_{16}$ and $\pi^{\star}\left(\mathrm{O}_{22}-\mathrm{H}_{22}\right)$ in isolated system and similarly for zwitterion it would be due to orbital overlapping between $\mathrm{LP}(2) \mathrm{O}_{21}$ and $\sigma^{\star}\left(\mathrm{N}_{16}-\mathrm{H}_{24}\right)$, which may cause intramolecular charge transfer (ICT) giving rise to the stabilization of the hydrogen bonded system [47]. Of course, the hydrogen bonding interaction leads to increase in the electron density $\left(\mathrm{E}_{\mathrm{D}}\right)$ of $\mathrm{O}_{21}-\mathrm{H}_{22}$ as well as $\mathrm{N}_{16}-\mathrm{H}_{24}$ non-Lewis orbital in the two species of L-TYR. Clearly, the energy values involved in the hyper conjugative interaction LP (1) $\mathrm{N}_{16} \rightarrow \pi^{\star}\left(\mathrm{O}_{21}-\mathrm{H}_{22}\right)$ and LP (2) $\mathrm{O}_{21} \rightarrow \sigma^{\star}\left(\mathrm{N}_{16}-\mathrm{H}_{24}\right)$ are 10.34 and $14.22 \mathrm{kcal} /$ mole. The other strong electron lone pair interactions are LP (1) $\mathrm{O}_{20} \rightarrow \mathrm{RY}^{*}$ (1) $\mathrm{C}_{19}, \mathrm{LP}(2) \mathrm{O}_{20} \rightarrow \sigma^{\star}\left(\mathrm{C}_{12-} \mathrm{C}_{19}\right)$, LP (2) $\mathrm{O}_{20} \rightarrow \sigma^{\star}\left(\mathrm{C}_{19} \mathrm{O}_{21}\right)$ and LP (2) $\mathrm{O}_{21} \rightarrow \pi^{*}\left(\mathrm{C}_{19}-\mathrm{O}_{20}\right)$ resulting in the stabilization energy of about 17.90, 19.05, 31.61 and $48.56 \mathrm{kcal} /$ mole in the isolated system while in zwitterion $\mathrm{LP}(1) \mathrm{C}_{4} \rightarrow \pi^{*}\left(\mathrm{C}_{2}-\mathrm{C}_{3}\right)$ and $\pi^{*}\left(\mathrm{C}_{5}-\mathrm{C}_{6}\right), \mathrm{LP}(2) \mathrm{O}_{20} \rightarrow \sigma^{\star}\left(\mathrm{C}_{12}-\mathrm{C}_{19}\right)$ and $\sigma^{*}\left(\mathrm{C}_{19}-\mathrm{O}_{21}\right), \mathrm{LP}(2) \mathrm{O}_{21} \rightarrow$ $\sigma^{\star}\left(\mathrm{C}_{12}-\mathrm{C}_{19}\right)$ and $\sigma^{\star}\left(\mathrm{C}_{19}-\mathrm{O}_{20}\right), \mathrm{LP}(3) \mathrm{O}_{21} \rightarrow \pi^{\star}\left(\mathrm{C}_{19}-\mathrm{O}_{20}\right)$ and LP(2) $\mathrm{O}_{22} \rightarrow$ $\mathrm{LP}^{\star}(1) \mathrm{C}_{1}$ possess stabilization energies $77.03,75.71,20.63,20.31,16.37$, $17.87,95.87$ and $49.41 \mathrm{kcal} / \mathrm{mole}$. It is interesting to note that in the present isolated molecular system, the non Lewis donor to non Lewis acceptor interactions $\pi^{\star}\left(\mathrm{C}_{1}-\mathrm{C}_{2}\right) \rightarrow \pi^{\star}\left(\mathrm{C}_{3}-\mathrm{C}_{4}\right)$ and $\pi^{\star}\left(\mathrm{C}_{5}-\mathrm{C}_{6}\right)$ exist with the higher interaction energies about 266.58 and 289.41respectively. Clearly, $\pi^{*}\left(\mathrm{C}_{1}-\mathrm{C}_{2}\right)$ a non Lewis NBO conjugated with the $\pi^{*}\left(\mathrm{C}_{5}-\mathrm{C}_{6}\right)$ resulting to the highest stabilization of $289.41 \mathrm{kcal} / \mathrm{mole}$.

\section{Conclusions}

The optimised structures of the most stable conformers of the L-TYR molecule in two different phases have been investigated. These are found to have $\mathrm{C}_{1}$ symmetry. The $\mathrm{r}\left(\mathrm{N}_{16}-\mathrm{H}_{24}\right)$ bond length have been found exceptionally larger and the bond angle $\alpha\left(\mathrm{N}_{16}-\mathrm{C}_{12}-\mathrm{C}_{19}\right)$ much shorter than the observed values in the zwitterion indicating the presence of intramolecular interaction. The natural, APT and Mulliken partial atomic charges at different sites of the lowest energy conformers in both the phases have been discussed. The $\mathrm{C}$ atom belonging to the $\mathrm{COOH} / \mathrm{CO}_{2}$ group possesses the largest magnitude of the APT atomic charge $(1.156077 \mathrm{e} / 1.677125 \mathrm{e})$ due to presence of the $\mathrm{O}_{20}$ atom. The $\mathrm{H}$ atoms of the $\mathrm{CH}_{2}$ group have the smallest magnitude $(0.001787 \mathrm{e} /$ $0.000283 \mathrm{e}$ ) for isolated and zwitterionic phases. The atoms $\mathrm{C}_{4}$ and $\mathrm{C}_{2}$ attain the highest and lowest values of the Mulliken atomic charges. The IR and Raman vibrational frequencies and the corresponding intensities of the most stable conformers of both the species have been computed using the DFT-B3LYP/ 6-311++ $\mathrm{G}^{* *}$ method. The computed vibrational fundamentals have been found to be in agreement with the experimentally observed frequencies. The values of the observed and the scaled wave numbers are close to each other. The presence of the IR band at $2075 \mathrm{~cm}^{-1}$ is an indicative of the presence of the molecule in the zwitterionic form. The intramolecular $\mathrm{H}$ bonding $\mathrm{O}_{21}-\mathrm{H}_{22}----\mathrm{N}_{16}$ in isolated form and $\mathrm{N}_{16}-\mathrm{H}_{24} \ldots \ldots . \mathrm{O}_{21}$ in zwitterion have been confirmed by the geometrical, vibrational and $\mathrm{NBO}$ analysis. The overlapping between the NBOs LP (1) $\mathrm{N}_{16}$ and $\pi^{*}\left(\mathrm{O}_{21}-\mathrm{H}_{22}\right)$ in the isolated form and between the NBOs LP (2) $\mathrm{O}_{21}$ and $\sigma^{*}\left(\mathrm{~N}_{16}-\mathrm{H}_{24}\right)$ in the zwitterionic form causes the intramolecular charge transfer (ICT) and also the stabilization of the $\mathrm{H}$ bonded system. The HOMO - LUMO energies reveal the ICT interaction between the donor and acceptor NBOs groups. The MEPs plots predict that in the isolated form of L-TYR the regions nearby each functional group $\mathrm{NH}_{2} / \mathrm{COOH} / \mathrm{OH}$ provide an active site for neucleo-philic attack, while in zwitterion $\mathrm{CO}_{2}$ group provide a vast region and $\mathrm{OH}$ group provide a site for neucleo-philic attack but no active region has been found in the neighbourhood of the $\mathrm{NH}_{3}$ group. The molecule is found to possess a permanent dipole moment and isotropic polarizability having the values 6.1104 Debye and 124.94 Bohr ${ }^{3}$ respectively in the isolated state but 12.99 Debye and $168.08 \mathrm{Bohr}^{3}$ respectively in the zwitterionic form. The material of the molecule is also found to be diamagnetic.

\section{References}

1. Bhowon MG, Jhaumeer-Laulloo S, Wah HLK, Ramasami P (2012) Chemistry for Sustainable Development, Springer Dordrecht Heidelberg, London, New York, pp: 81-102.

2. Skerget M, Kotnik P, Hadolin M, Hras AR, Simonic M, et al. (2005) Phenols, proanthocyanidins, ?avones and ?avonols in some plant materials and their antioxidant activities. Food Chem 89: 191-198.

3. Gulcin I (2007) Comparison of in vitro antioxidant and antiradical activities of L-tyrosine and L-Dopa. Amino Acids 32: 431-438.

4. Kecel S, Ozel A E, Akyuz S, Celik S, Agaeva G (2011) Conformational analysis and vibrational spectroscopic investigation of L-proline-tyrosine (L-Pro-Tyr) dipeptide. J Mol Str 993: 349-356.

5. Ramaekers R, Pajak J, Rospenk M, Maes G (2005) Matrix-isolation FT-IR spectroscopic study and theoretical DFT(B3LYP)/6-31++G** calculations of the vibrational and conformational properties of tyrosine. Spectrochim Acta A Mol Biomol Spectrosc 61: 1347-1356.

6. Contreras CD, Ledesma AE, Lanus HE, Zinczuk J, Brandan SA (2011) Hydration of I-tyrosine in aqueous medium. An experimental and theoretical study by mixed quantum mechanical/molecular mechanics methods. Vibrational Spectroscopy 57: 108-115

7. Gelenberg AJ, Gibson CJ, Wojcik JD (1982) Neurotransmitter precursors for the treatment of depression. Psychopharmacol Bull 18: 7-18.

8. Meyer JS, Welch KM, Deshmukh VD, Perez FI, Jacob RH, et al. (1977) Neurotransmitter precursor amino acids in the treatment of multi-infarct dementia and Alzheimer's disease. J Am Geriatr Soc 25: 289-298.

9. Deijen JB, Wientjes CJ, Vullinghs HF, Cloin PA, Langefeld JJ (1999) Tyrosine improves cognitive performance and reduces blood pressure in cadets after one week of a combat training course. Brain Res Bull 48: 203-209. 
Citation: Yadav RA, Dixit V, Yogesh M, Santhosh C (2015) Raman and IR Spectral and DFT Based Vibrational and Electronic Characterization of Isolated and Zwitterionic Forms of L-Tyrosine. Pharm Anal Acta 6: 439. doi:10.4172/21532435.1000439

10. Mongan J, Case DA, McCammon JA (2004) Constant pH molecular dynamics in generalized Born implicit solvent. J Comput Chem 25: 2038-2048.

11. Fang L, Zhang H, Cui W, Ji M (2008) Studies of the mechanism of selectivity of protein tyrosine phosphatase 1B (PTP1B) bidentate inhibitors using molecular dynamics simulations and free energy calculations. J Chem Inf Model 48: 20302041.

12. Hu X, Stebbins CE (2006) Dynamics of the WPD loop of the Yersinia protein tyrosine phosphatase. Biophys J 91: 948-956.

13. Mostad A, Nissen HM (1971) The structure of I-tyrosine. Tetrahedron Lett 12 2131-2132.

14. Frey MN, Koetzle TF, Lehmann MS, Hamilton WC (1973) Precision neutron diffraction determination of protein and nucleic acid components. I. The crystal and molecular structure of the amino acid L-alanine. J Am Chem Soc 58: 2657 2660.

15. Goodwill KE, Sabatier C, Marks C, Raag R, Fitzpatrick-Raymond PF, et al. (1997) Crystal structure of tyrosine hydroxylase at $2.3 \AA$ and its implications for inherited neurodegenerative diseases. Nat Struct Biol 4: 578-585.

16. Antson AA, Demidkina TV, Gollnick P, Dauter Z, von Tersch RL, et al. (1993) Three-dimensional structure of tyrosine phenol-lyase. Biochemistry 32: 41954206.

17. Yang J, Liu L, He D, Song X, Liang X, et al. (2003) Crystal structure of human protein-tyrosine phosphatase SHP-1. J Biol Chem 278: 6516-6520.

18. Mohammadi M, Froum S, Hamby JM, Schroeder MC, Panek RL, et al. (1998) Crystal structure of an angiogenesis inhibitor bound to the FGF receptor tyrosine kinase domain. EMBO J 17: 5896-5904.

19. Suga T, Inubushi C, Okabe N (1998) O-Phospho-L-tyrosine. Acta Crystallogr C 54: 83-85.

20. Broermann A, Steinhoff HJ, Schlücker S (2014) Towards label-free and sitespecific probing of the local $\mathrm{pH}$ in proteins: $\mathrm{pH}$-dependent deep UV Raman spectra of histidine and tyrosine. J Mol Str 1073: 77-81.

21. Wojciechowska A, Kochel A, Komorowska M (2011) Synthesis, crystal structure and spectroscopy studies of a novel five-coordinated $\mathrm{Zn}$ (II) ion complex with L-tyrosine and imidazole. Polyhedron 30: 2361-2367.

22. Fischer WB, Eysel HH (1997) Raman and FTIR spectroscopic study on water structural changes in aqueous solutions of amino acids and related compounds. J Mol Str 415: 249-257.

23. Grace LI, Cohen R, Dunn TM, Lubman DM, de Vries MS (2002) The R2P Spectroscopy of Tyrosine: A Vibronic Analysis. Journal of Molecular Spectroscopy 215: 204-219.

24. Ojha AK (2007) pH dependent SERS and solvation studies of tyrosine adsorbed on silver colloidal nano particles combined with DFT calculations. Chemical Physics 340: 69-78.

25. Dixit V, Yadav RA (2015) Experimental IR and Raman Spectroscopy and DFT Methods Based Material Characterization and Data Analysis of 2- Nitrophenol. Biochem Pharmacol (Los Angel) 4: 183.

26. Frisch MJ, Trucks GW, Schlegel HB, Scuseria GE, Robb MA, et al. (2010) Gaussian 09, Revision C.01, Gaussian, Inc., allingford, CT.

27. Dixit V, Yadav RA (2015) DFT-B3LYP computations of electro and thermo molecular characteristics and mode of action of fungicides (chlorophenols). Int J Pharm 491: 277-284.

28. Dixit V, Yadav RA (2015) Structural and vibrational studies of some pesticides: 2-chlorophenol,2,4,6-trichlorophenol and pentachlorophenol. Asian Journal of Physics 24.

29. Martin JML, Van Alsenoy C (1995) GAR2PED, A Program to Obtain a Potential Energy Distribution from a Gaussian Archive Record, University of Antwerp, Belgium.

30. Rauhut G, Pulay P (1995) Transferable Scaling Factors for Density Functional Derived Vibrational Force Fields. J Phys Chem 99: 3093-3100.

31. Govindarasu K, Kavitha E (2014) Molecular structure, vibrational spectra, NBO UV and firrst order hyperpolarizability, analysis of 4-Chloro-DL-phenylalanine by density functional theory. Spectrochimica Acta Part A 133: 799-810.

32. Dewar MJS (1969) The Molecular Orbital Theory of Organic Chemistry. McGraw-Hill and Inc.
33. Charles Alfred Coulson, McWeeny R (1979) Coulson's Valence. Oxford University Press.

34. Ferreira MMC, Suto E (1992) Atomic polar tensor transferability and atomic charges in the fluoromethane series. J Phys Chem 96: 8844-8849.

35. Leyton P, Brunet J, Silva V, Paipa C, Castillo MV, et al. (2012) An experimental and theoretical study of L-Tryptophan in an aqueous solution, combining two-layered ONIOM and SCRF calculations. Spectrochim Acta A Mol Biomo Spectrosc 88: 162-170.

36. Dixit V, Yadav RA, Yogesh M, Santhosh C (2015) Experimental IR and Raman and DFT methods based spectroscopic and electronic chracterization of L-phenylalanine in isolated and zwitterionic forms. Vib Spectrosc.

37. Gunasekaran S, Balaji RA, Kumaresan S, Anand G, Srinivasan S (2008) Experimental and theoretical investigations of spectroscopic properties of $\mathrm{N}$-acetyl-5-methoxytryptamine. Can J Anal Sci Spectrosc 53: 149-160.

38. Foresman JB, Frisch AE (1996) Exploring Chemistry with Electronic Structure Methods (2nd edn) Gaussian, Pittsburgh, PA.

39. Lewars E (2003) Computational Chemistry-Introduction to the Theory and Applications of Molecular and Quantum Mechanics, Kluwer Academic Publishers, Norwell, MA

40. Manolopoulos DE, May JC, Down SE (1991) Theoretical studies of the fullerenes: C34 to C70. Chem Phys Lett 181: 105-111.

41. Politzer P, Truhlar D (1981) Chemical Application of Atomic and Molecular Electrostatic Potentials, Plenum, New York pp: 257-294.

42. Moro S, Bacilieri M, Ferrari C, Spalluto G (2005) Autocorrelation of molecular electrostatic potential surface properties combined with partial least squares analysis as alternative attractive tool to generate ligand-based 3D-QSARs. Curr Drug Discovery Technol 2: 13-21.

43. Murray JS, Sen K (1996) Molecular Electrostatic Potentials: Concepts and Applications, Elsevier, Amsterdam.

44. Weiner PK, Langridge R, Blaney JM, Schaefer R, Kollman PA (1982) Electrostatic potential molecular surfaces. Proc Natl Acad Sci U S A 79: 3754 3758.

45. Politzer P, Truhlar DG (1981) Chemical Application of Atomic and Molecular Electrostatic Potentials, Plenum, New York.

46. James C, Amalraj A, Reghunathan R, Joe IH, Jayakumar VS (2006) Structura conformation and vibrational spectroscopic studies of 2,6-bis(p-N,N-dimethyl benzylidene) cyclohexanone using density functional theory. J Raman Spectrosc 37: 1381-1392.

47. Liu JN, Chen ZR, Yuan SF (2005) Study on the prediction of visible absorption maxima of azobenzene compounds. J Zhejiang Univ Sci B 6: 584-589. 